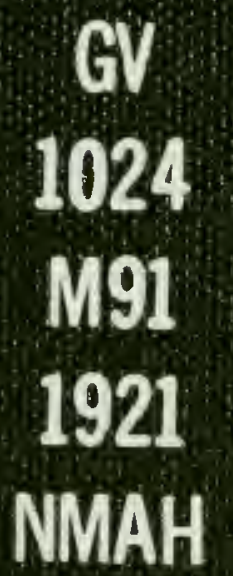






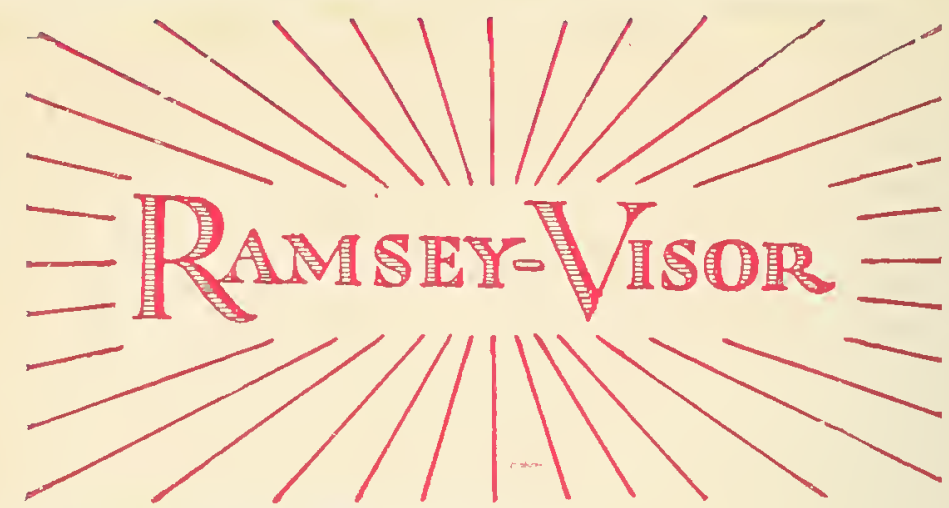

IT PROTECTS YOU-

\section{At a Trifling Cost}

-from the Dangers of Driving at Night, shielding your eyes from the blinding glare of high-powered headlights, spot-lights, arc-lights, electric signs, etc.

-from the accumulation of Rain, Snow and Sleet upon your windshield, which obstructs your vision of pedestrians, vehicles, etc.

-from Sun Glare and Road Glare and consequent tired, dazzled eyes and over-burdened nerves.

-from "Driver's Headache" and the "Automobile Stare" - and adds immeasureably to the safety and comfort of yourself and your passengers.

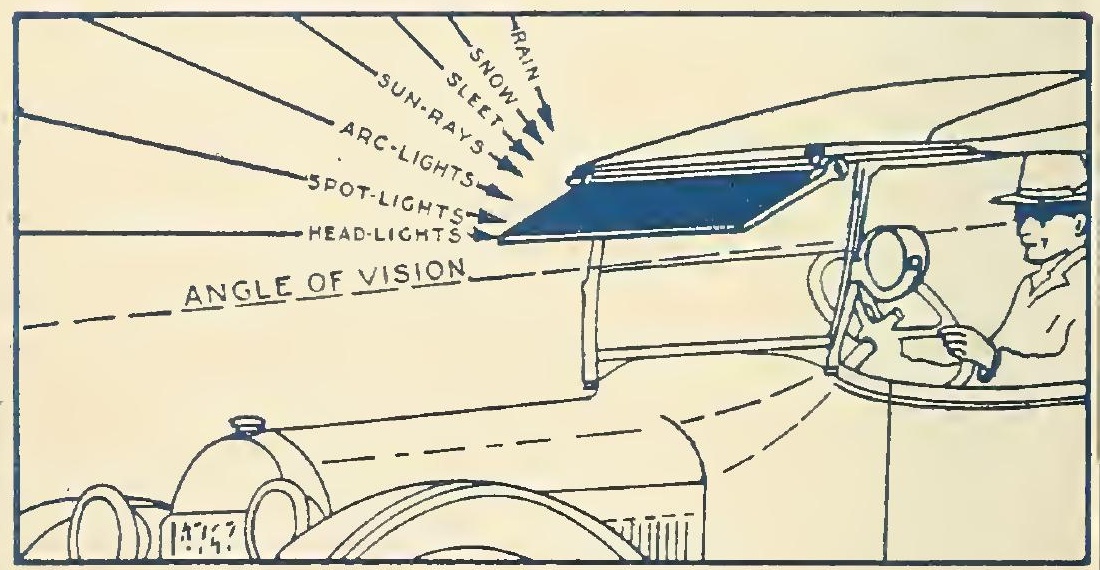

Adjustable for any angle. Made for open and closed cars. Steel frame-Fabric leather cover.

"SIMPLEX" Mø̈det, $10 "$ x $40 " \ldots \ldots \ldots \ldots$. Price $\$ 7.00$

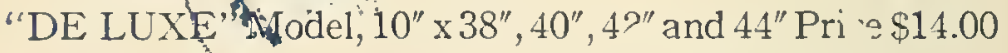

CDECII ACECD. Send us remittance together SPEAL UFTER. with the name, year and model of your car and we will ind you a RAMSEYVISOR. Try it for five days and if you are not perfectly satisfied return it to us and we will refund your money.

Manufacture ny

THE ALBANY MACHINE \& TOOL CO. 1033 BROADWAY, ALBANY, N. Y. Dealers Wanted-Write for Proposition 


\title{
MOTOR TOURS
}

The Guide all Tourisss need Will save you hundreds of dollars

\author{
Rumning Directions \\ Sectional Maps \\ Suggestions of Value
}

NEW YORK STATE, NEW ENGLAND STATES, CANADA, PENNSYLVANIA, OHIO, INDIANA, MICHIGAN, WASHING $=$ ATLANTIC CITY

\section{PRICE 50 GENTS}

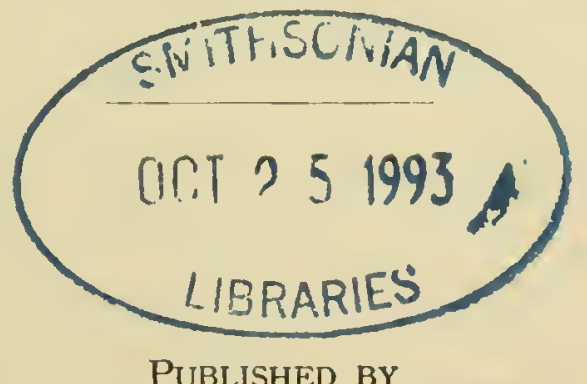

AMSTERDAM ADVERTISING CO.

AMSTERdAM, N. Y.

Copyright 1921, Amsterdam Advertising Co. 


\section{INDEX}

Albany, N r to PAgE

Amsterdam, N. Y Binghamton, N. Y....... 5 Greenfield, Mass. ....... 94 Kingston, N. Y......... 13 Pittsfield, Nass. ........ 83 Poughkeepsie, N. I...... 11 Rutland, Vt. ......... 97 Saratoga, N. I......... 1 Troy, N. Y........... 13

Alexanulia Bay, N. Y., to Rouses Point, N. Y..... 63 Syracuse, N. Y......... (it Utica via Cape Vincent \& Watertown ........... oc

Altoona, Pa., to Williamsport, Pa. ...... 134

Amagausett, I. I., to New York, N. Y........ 7

Amsterdam, N. Y., to Albany, N, Y Broadalbiu, $\mathbf{N} . \quad \mathrm{Y} \ldots \ldots \ldots$, 32 Lake Pleasant .......... 32 Utica, N. Y............ 28

Atlantic City, N. J., to New York, N. Y......... so Phlladelphia, Pa. ...... 126

Baltimore, Md., to Philadelphia, I'a. ....... 78 Washington, D. C........ 78

\section{Bangor, Me., to} Poland Spring, Ne..... 105 Portland, Me. ........ 102

Binghamton, N. Y., to Albauy, N. Y......... 50 Glmira, N. Y $. \ldots \ldots \ldots \ldots, 48$ New York, N. Y....... + $8-52$ Scranton, I'a. ..........130 Utica, N. Y.............

Boston, Mass., to Concord, N. H........... s Falmouth, ilass. .......... Greenfield, Mass. ......... Portland, MIe. ........... 10 Providence, R. I....... 05 Provincetown, MIass. .... 128 Worcester, Miss. ....... 80

Bretton Woods, N. II., to Kieene, N. H........... 110

Bridgeport, Coun., to New Haven ............ 90 New York .............. 88

Broadaloin, N. Y., to Amsterdam, N. $Y \ldots \ldots \ldots$. 82

Buffalo, N. Y., to Dansville, N. Y....... T1 Erie, Pa. ............ 4 Niagava Falis ........... T1 Olean, N. Y......... 4 . Rochester, $N$. Y.......... 30 Syracuse, N. Y......... 39

Burlington, Vt., to Rutland, vt. ........... 9? White Mountains, N. H.. 98

Chestertown, N. Y., to Long Lake, N. Y........ 2t

Chicago, 111., to Kalamazoo Mich, ....... 131 South Bend, Ind........ 198

Cleveland, Ohio, to Erie, Рa. ............ 40 Toledo, ohio ............. 123

Concord, N. H., to Boston, Mass. ......... 101 Profile Honse ........... 100

Cooperstown, N. Y., to Little Falls, N. Y....... 51 Watkins, N. Y.......... 50
Dansville, N. Y., to Page Buffalo, N. Y........ 71 Elmira, N. $\mathbf{Y} \ldots \ldots \ldots \ldots \ldots, 60$

Detroit, Mich., to Kalamazoo, Mich ....... 124 London, Ont. .........123 Toledo, Onto .........131-132

Delaware Water Gap, Pa., to New Tork City........ 141 Scranton, Pa. ......... 141 Wilkes-Harre, Pa. ....... 139

Dubois, Pa., to Olean, N. Y......... 43 Pittsburgh, Pa. ........ 139

Easton, Pa., to Philadelphia, $\mathrm{Pa} . \ldots \ldots .136$ Port Jervds, N. Y..... 136

Ellzabetitown, N. Y., to Lake George, N. Y...... 20 Malone, N. I............ 23 Plattsbnrg, N. $\mathrm{Y} \ldots \ldots \ldots, 21$

Elmira, N. Y., to Binglamton, N. Y...... 60 Dausville, N. Y....... 60 Olean, N. I.......... 49 Syracuse, N. Y........ 54 WVatklns, N. Y........ 46 Wtlliamsport, Pa. ..... 135

Crie, Pa., to Buffalo, N. Y.......... 36 Cleveland, Ohio ........ 40 Pittsburgh, Pa. ........ 138

Falmonth, Mass., to Boston, Mass. ........ 93

Fonda, N. Y., to Isake Pleasaut, N. Y.... 30 Geneva, N. Y., to Watkins, N. Y......... 52 Greenfield, Mass., to Albany, N. Y......... 92 Boston, N. Y .......... 92 Greenport, I. I., to New York, N. Y...... 72

Harrisburg, Pa., to Philadelphta, Pa. ...... 135 Washington, D. C...... 137 IVilliamsport, Pa. ...... 137

Ilartford, Conn., to New Haven, Conn...... 82 Sinringfield, Mass. ...... 126

IIornell, N. Y., to Iiochester, N. Y .......,69

ithaca, N. Y., to Watkins, N. Y........ 46

Kalamazoo, Mich., to Chicago, $111 . \ldots \ldots \ldots \ldots .124$ Detroit. Nich. .......... 131

lieene, N. HI, to

Springfield, Mass. ...... 108 White Mountains, $\dot{N} . \dot{H} . .110$

Kingston, N. Y., to Oneonta, N. I........ 51 New York City........... 12 Albany, N. Y......... 13

Kingston, Canada, to Toronto, Ont. ......... 116 Montreal, P. Q.......... 118

Lake George, N. Y., to Elizabethtown, N. Y.... 20 Saratoga Springs, N. Y.. 22 Saratoga via Lake Luzerne $. . \ldots \ldots \ldots \ldots \ldots, 17$ Westport, N. Y........ 18

Lake Pleasant, $N$. Y., to Amsterdam, N. Y....... 32 Fonda, N. Y.......... 30 
Little Falls, N. F., to Page Cooperstown, N. I..... 50

Long Lake, $N$. Y., to Chestertown, N. I..... 66

London, Canada, to Toronto, Canada ....... 114 Detroit, Mich. ........ 123

Malone, N. F., to

Elizabethtown, N. Y.... 24

Iontreal, P. Q., to

Plattsburg, N. Y ....... 26

Kingston, Ont. .......... 118

Quebec. Canada ........ 120

New Haven, Conn., to Bridgeport, Conn. ....... 90 Hartford, Conn. ....... 82 New London, Conn. ..... 88

New London, Conn., to New Haren, Conn....... 91 Providence, R. I........ $8 \mathrm{~S}$

New York, N. Y., to A magan sett, I. I...... 74 Atlantic Cits, N. J..... 80 Binghamton, N. I...... 44 Bridgeport, Conn. ...... 88 Greenport, I. I......... T2 Kingston, $N . \quad$ I......... 12 Philadelphia, Pa. ....... 75 Poughkeepsie, N. Y..... 8 Delaware Water Gap, Pa 141

Niagara Falls, N. Y., to Buffalo, N. Y......... T1 Rochester, $\mathbf{N}$. $\ldots \ldots \ldots . . .68$ Toronto, Can. ......... 111

Olean, N. Y., to Buffalo, N. I. . . . . . 49 Dubois, Pa. .......... 43 Elmira, N. $\dot{Y} \ldots \ldots \ldots \ldots \ldots .49$ Westfield, N. Y........ 47

Oneonta, $N$. $Y_{\text {., to }}$ Kingston, N. $\mathrm{Y} \ldots \ldots \ldots \ldots, 51$

Oswego, $\mathbf{N}$. $\mathbf{Y}$., to Syracuse, N. Y........69

Ottawa, Canada, to Prescott, Canada ....... 122

Plattsburgh, N. Y., to Elizabethtomn. N. Y.... 22 Montreal, P. Q........ 25

Philadelphla, Pa., to Atlantic City, N. J ..... 80 Baltimore, Ma. ....... 75 New York, N. I.......... 76 Harrisburg, Pa. ...... 135 Easton, Pa. ........... 136

Pittsburgh, Pa., to Frie, Pa. ............ 138 Dubois, Pa. ........... 139 Warren, Pa. .......... 140

Pittsfleld, Mrass., to Albany, N. Y......... 84 Rutland $v$ t. ............... 100 Springfield, Mass. ...... \$.3

Poland Springs, Me., to Bangor, Ne. .......102-105

Portland, Mre., to Bangor, Me. ........101-102 Boston, Mass. ........... 104 White Mowntains, $\ddot{N}$. $\ddot{H}$. 98

Port Jerria, N. Y., to Easton, Pa. ........... 130

Pouglikeepsie, N. Y., to New York Cits ........ 10 Alban $\ldots . \ldots \ldots \ldots \ldots \ldots . . . .11$

Prescott, Canada, to Ottawa, Canada ....... 122

Provlncetown, Mass., to Boston, Mass. ......... 12s

Profle House, N. H., to Concord, N. H........ 100

Providence, R. I., to Boston, Mass. ......... 9: New London, Conin...... i5

Quebec, Canada, to Iontreal, Canada
Rochester, N. Y., to PAGE Buffalo, N. Y ......... 3 Hornell, N. Y......... 69 Niagara Falls, N. I.... 67 Syracuse, N. Y.......34-35 Watertown, $\mathbf{Y} \ldots \ldots . . .70$

Rouses Point, N. Y., to Alexandria Bay, N. I... 63

Rutland, Vt., to Albany, N. Y......... 99 Burlington, $\mathrm{Vt}$. ....... 97 Pittsfield, Mass. ........ 107

Saratoga Springs, N. Y., to Lako George via Lake Luzerne ............. 1T Albany, N. $I_{\ldots \ldots \ldots \ldots \ldots .1}$ Lake George, N. I...... 16

Scranton, Pa., to

Wilkes Barre, Pa. ..... 42 Binghamton, $N$. I...... 136 Waveriy, N. Y......... 138 Delaware Water Gap, Pa. 141

South Bend, Ind., to

Toledo, Ohlo .......... 132 Chicago, IIl. .......... 139

Springfleld, Mass., to

Hartford, Conn. ....... 82 Keene, N. H........... 108 Pittsfield, Mass. ....... 84 Worcester, Mass. ...... 87

Syracuse, N. $\mathbf{Y}$, to

Buffaio, $N . \quad Y \ldots \ldots \ldots \ldots .38$ Elmira, N. $Y \ldots \ldots \ldots \ldots .54$ Oswego, N. I......... 65 Rochester, N. $\mathrm{I} \ldots \ldots \ldots \ldots 34$ Utica, N. Y .......... 33 Watertown, N. Y....... 65 Alexandria Ba. ........ 129

Toronto, Canada, to

Niagara Fails, N. I. . . 112 London, Canada ........ 114 Kingston, Canada ....114-116

Toledo, Ohio, to Cleveland, Ohio ........ 120 South Bend, Ind... . . 132-133 Detroit, Mich. ......... 132

Troy, N. Y., to Albany, $N . \quad T \ldots \ldots \ldots 13$

Utlea, N. Y., to

Amsterdam, N. I....... 28

Binghamton, N. Y..... 55 Stracuse, N. Y........ 33 Watertown \& Alexandria Bay .............. 62

Washington, D. C., to Baltimore, Md. ....... 78 Harrisburg, Pa. ....... 137

Watertown, N. Y.., to Rochester, N. $Y \ldots \ldots \ldots$ T0 Syracuse, N. Y........ 65

Warron, Pa., to Pittsburgh, Pa. ........ 140

Watkins, N. Y., to Cooperstomn, N. I..... 50 Fimira, N. Y .......... 46 Geneva, N. Y.......... 52 Ithaca, $N . \mathbf{Y} \ldots \ldots \ldots \ldots \ldots 46$

Warerly, N. $I_{\text {., to }}$ Scranton, Pa. ........ 134

Westfleld, N. Y., to Olean, $N$. Y.......... 47

Westport, N. Y., to Lake George, N. Y..... 19

White Mountalns, N. H., to Burlington, Vt. ....... 99 Portland, Mte......... 98

IVillamsport, Pa., to Altoona, Pa. ......... 134 Elmira, N. Y........... 135 Harrisburg, Рa. ........ 137

wilkes-Barre, Pa., to Scranton, Pa. ......... 80 Delaware Water Gap, Pa. 140

Vorcester, Mrass., to Boston Mass ......... 96 Springfield, Mass. ...... 86 


\section{IN PHILADELPHIA, IT'S THE MAJESTIC}

On the LIncoln IIghway at Broad St. and Girard Ave.

500 rooms with bath, single or ensuite

Superior Cafe and Grill Rooms

The rates are surprisingly moderate in view of the many unduplicated advantages of the Majestic

GARAGE FACILITIES

Management of - - - CHARLES DUFFY,Jr.

\section{The O-TE-SA-GA}

On the Historical Otsego Lake

OPENS JUNE 18

COOPERSTOWN, N. Y.

\section{NEW CUMBERLAND}

OFFICIAL TOURISTS HOTEL

American Plan

First Class Appointments.

R. J. CLARK, Prop.
Rates $\$ 4.50$ and $\$ 5.00$ with Bath

Elevator. Hot and Cold Water in All Rooms PLATTSBURGH, N. Y.

\section{When in Oneonta}

\section{Eat at---KILKENNY'S}

\section{Hotel Seneca GENEVA, N. Y.}

The Gem of the Finger Lakes Region. European Plan. 100 Rooms with Bath. Restaurant and Grill made famous

by Seneca Lake Trout. Service a la Carte and Table de Hote. Grill open till Midnight

GLENN R. MORTON, Manager

\section{STOP AT...}

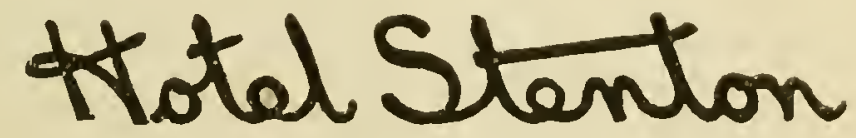

Broad ${ }^{-a n d}$ Spruce Streets

PHILADELPHIA, PA.

Ideal Location

Fireproof

REASONABLE RATES 


\section{Ask Mr. Fostor}

to map out your automobile trips for you. $\mathrm{He}$ can give you personal information about the best trips, roads and inns and hotels enroute. Personally supervised tours are best.

Also ask for information regarding camps, walking tours, horseback trips, resort hotels, city hotels, railroad schedules and all information relative to travel. No fees.

\section{Ghe \\ Ask Mr. Foster Travel Information Service}

Forty offices in large cities and resorts

\section{Executive Offices}

130 WEST 42nd STREET NEW YORK CITY

\section{N I A GARA I N N}

Near the Falls

Buffalo Ave.,

Niagara Falls, N. Y. SPEGIAL DINNERS

LOBSTER

FISH

CHICKEN

STEAKS

\section{HENRY NEFF}

\section{INDEX OF MAPS}

Page

New York City

New York to Albany.......

Albany, N. Y., to Montreal,

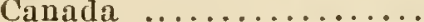

Albauy, N. Y., to Syracuse,

N. Y. $\dot{Y}^{\prime} \cdots \cdots$ to Lake

Albany, N. Y., to Lake

Pleasant, N. Y., to Buffalo.

Syrachse, N. I., to Buffalo, N. Y. $\cdots$ N. $\because$. to Buffalo,

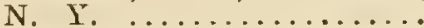

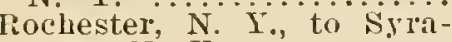
cuse, N. ${ }_{\text {Y }} \ldots \ldots \ldots \ldots$ to Buffalo, N. Y., to Toledo, Ohio

New York to Binghamton.

Elmira, N. Y., to New York

Syracuse, N. Y., to Elmila

Albany, N. Y., to Eingham-

ton, N. Y........... 5

Utica, N. Y., to Bingham-

ton, N. Y.............

Utica, N. Y., to Alexandria

Bay, $N$. $Y . \ldots \ldots \ldots \ldots$
New Fork to Philadelphia To

Philadelphia to Washing-

ton ............... 79

New York to Atlantic City,

N. J. ............ 81

Albany, Mass. ................ 85

New York to lioston Mass. 89

boston, Mass., to Bangor,

Me. ................. 103 Boston to White Mountains 109 Buffalo to Detroit, Mich., via Canada ........... 115 Ottawa, Can., to Toronto, Canada ................ 117 Quebec to Ottarva, Canada. 121 Montreal to Quebec ....... 121 Chicago to Detroit, Mich.. 125 Philadelphia to Atlantic City, N. J.......... 127 Montieal to Rochester..... 143 Ariirondack Mountains .... 143 Bingluamton to Buffalo.... 142 Syracuse to Buffalo...... 142 Montreal to Kingston..... 143 


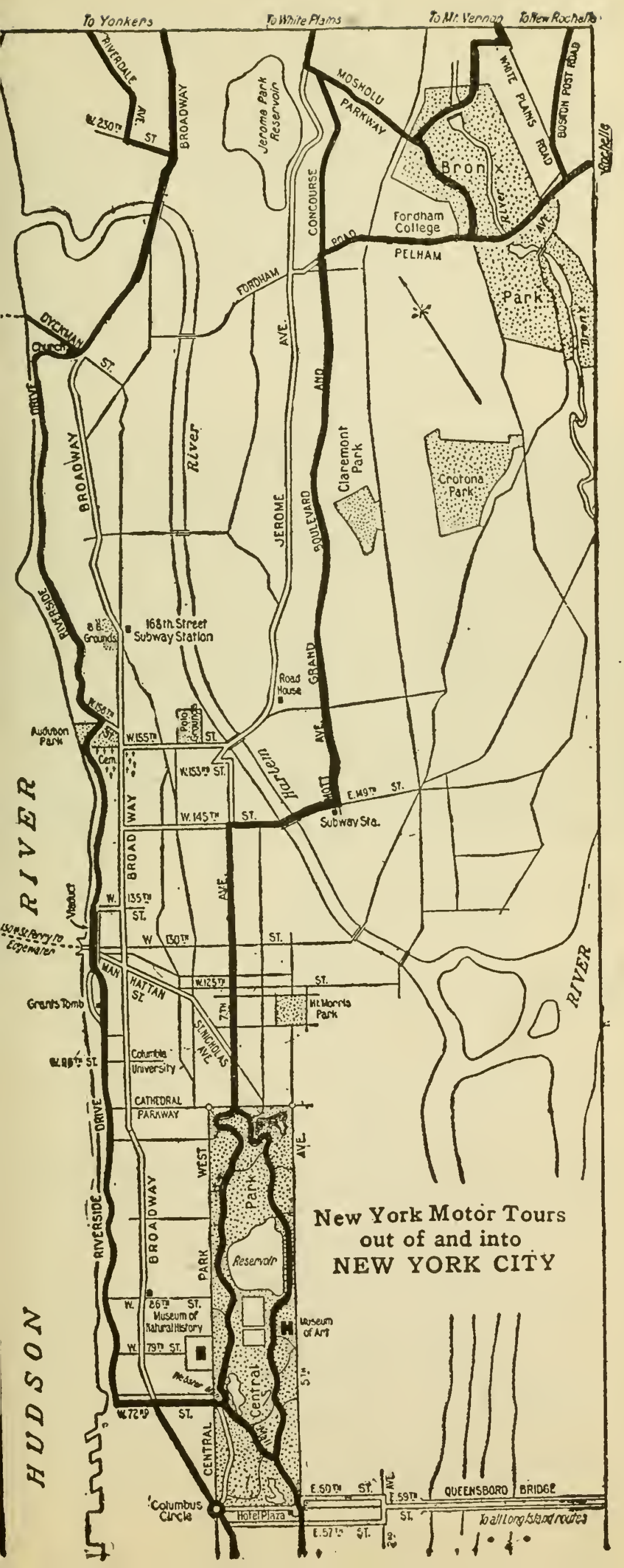


New York to Poughkeepsie, N. Y., 72.8 Miles

0.0 From Automobile Club of New York, Hotel Ansonia West on 74th St.

0.2 Right on Riverside Dr.; keep L at 5 cors. 4.4.

7.2 Left at 5 cors. on Broadway to Yonkers.

12.8 Bear left across Getty Sq.; at once right.

10.0 Left at 4-cors. Dobbs Ferry, thru Tarrytown.

22.0 Left fork at church; I at mon. Ossining 30.6.

31.2 Left ar. school; thru Croton 33.3; right 38.2.

38.6 Right with trolley; left 38.9 ; right at end.

40.8 Left on Division St. Peekskill; left fork 41.2.

45.7 Right fork; right at hotel, Fishkill thru. Left fork 45.7 leads to Beacon and Ferry for Newburgh.

64.8 Wappingers Falls to end of Market St.

72.8 Poughkeepsie, Market St. at Main.

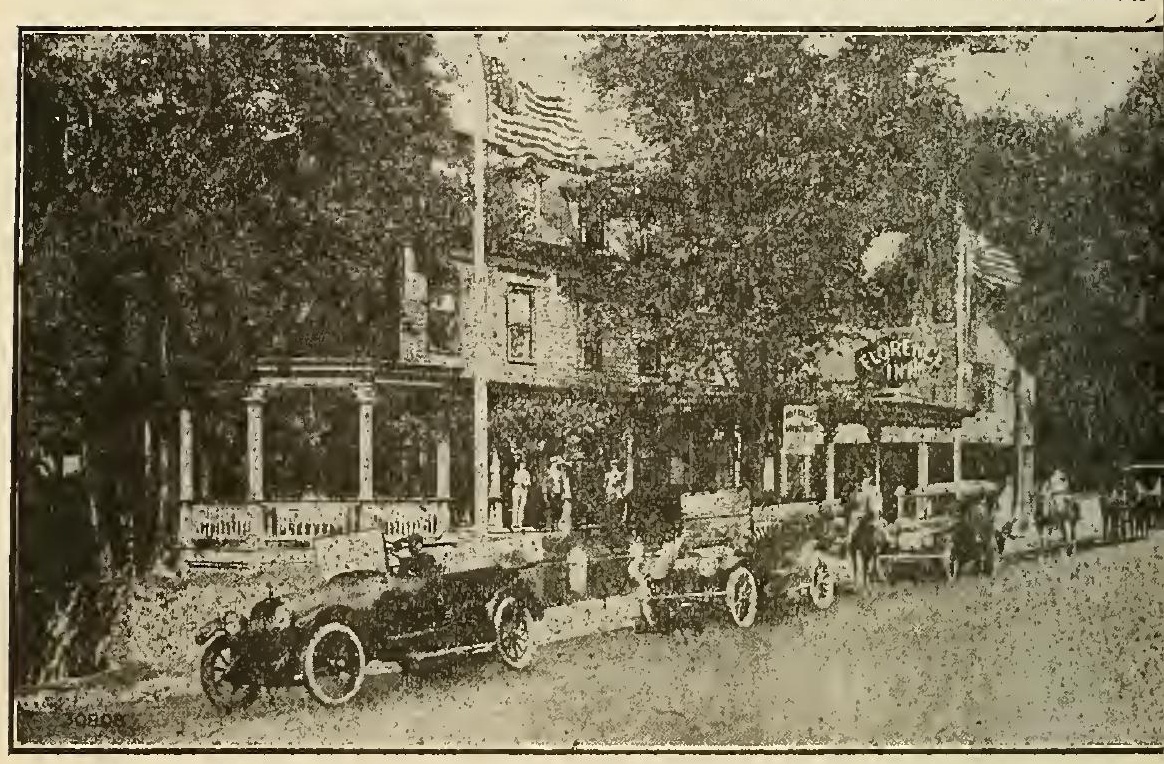

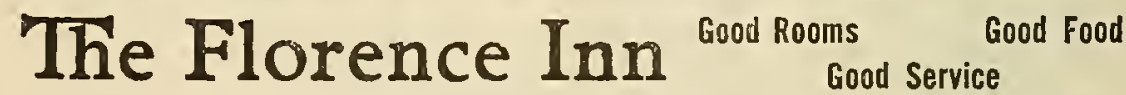

GEO. F. PARKE, Tarrytown, N. Y.

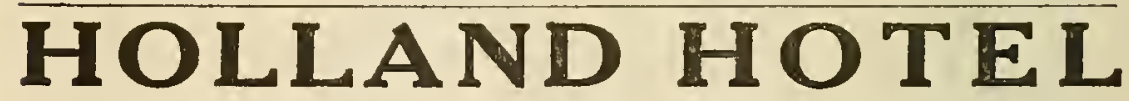

J. B. Hyatt, Manager

60 rooms

BEACON, NEW YORK

European $\$ 2.00$ up

Many private baths

American $\$ 5.00$ per day up

Famous Cuisine

WHILE IN... Poughkeespie

stop at VAN'S GARAGE, one of the oldest and most reliable establishments of its kind

We are agents for the

Marmon, Peerless, Studebacker and Dodge Brothers motor cars, also the Mack truck

LET US SERVE YOU

PHONE 1220

JOHN VAN BENSGHOTEN, Inc.

12-24 Catharine St.

Poughkeepsie, N. Y. 


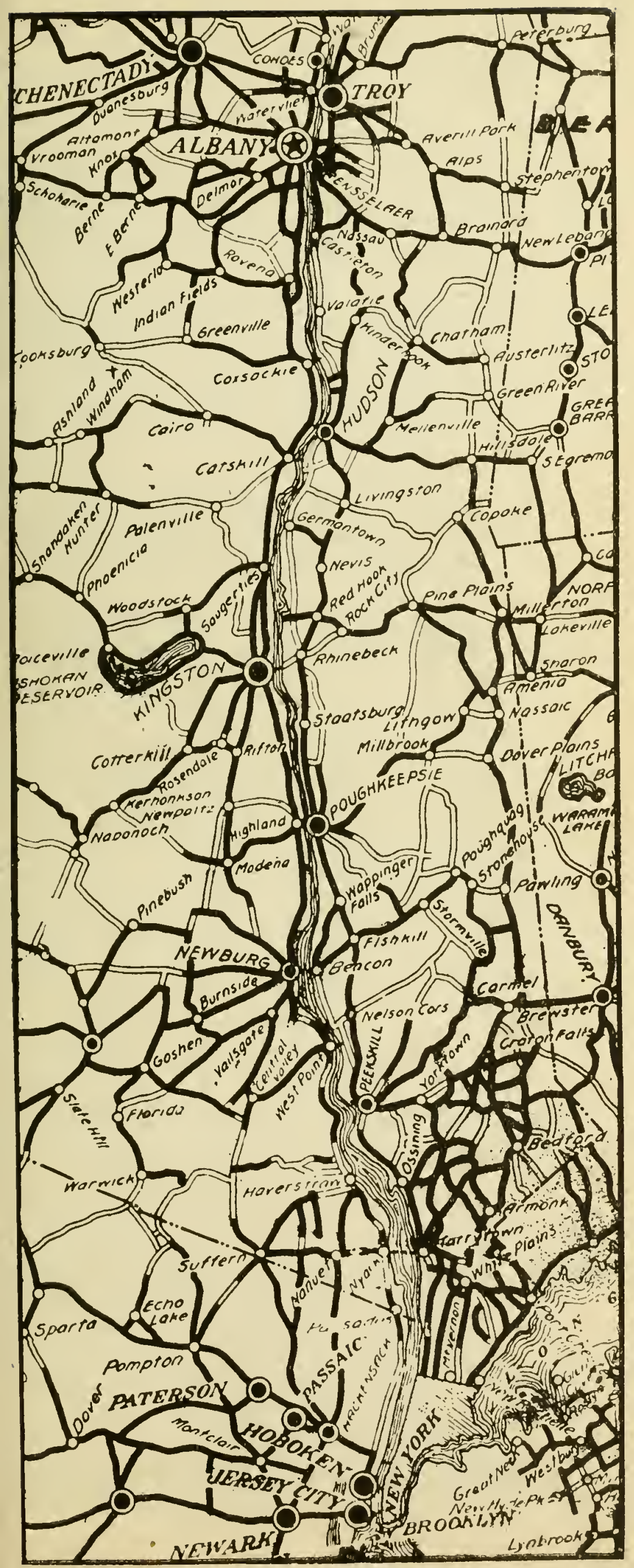


Poughkeepsie to New York, N. Y., 72.8 Miles

0.0 From Main St. Poughkeepsie, south on Market.

0.3 Left fork; thru Wappingers Falls 8. to Fishkill.

12.6 Left at end; right fork 18.7 into Peekskill.

32.0 Right on South St.; 2nd left; right with trol.

33.8 Left thru Croton 39.5; right at end 41.7 thru.

42.3 Ossining and Tarrytown 49.5 into Dobbs Ferry.

53.8 Right at trough; left fork; left fork 55.2.

60.0 Bear left across Sq. Yonkers; at once right.

65.6 Half right on Riverside Dr.; half right 68.4.

72.6 Left on 74th St. two blocks.

72.8 Automobile Club of New York, Hotel Ansonia, on right.

The Home of the Motorist when in New York City

The Holley Hotel

Washington Square

Facing the beautiful Washington Square Park, away from the noise and hustle of the busy city, adjoining the heart of the shopping and theatrical district.

Rates Most Reasonable

American or European Plan Excellent Garage Accommodations

UNDER KNOTT MANAGEMENT

SHERMAN K. HILL, Manager

PHONE SPRING 3309

\section{Putnam Auto Salvage \& Repair Corp.}

178 Glove Ave., Haverstraw, N. Y. Telephone, Haverstraw 139-L-4

New York Office

302 Broadway

Telephone, Worth 0444

See Advertisement page 107

We Buy Wrecked Cars 
Poughkeepsie to Albany, N. Y., 75.6 Miles

0.0 From Market St., Poughkeepsie W. on Main.

0.1 Right on Washington, under R.R. direct to Rhinebeck.

17.2 Right fork; thru Red Hook 22.; Blue Stores 31.

33.9 Right thru Livingston; right fork 39.8.

42.4 Half right on Prospect St. at 5 cors., Hudson.

42.7 Right at end: next left with trol.; to Stockport.

48.2 Half left at garage; thris Kinderhook 55.

56.9 Left at sta. Valatie; half right at 4-cor. 57.7.

74.4 Right on Broadway, Rensselaer; left across Hudson River.

75.1 Right on Pearl St. with trolley.

75.6 Albany, State St., at Pearl St.

\section{Beekman Arms RHINEBEGK, N. Y.}

OPEN MAY TO NOVEMBER

W. W. FOSTER : : : PROPRIETOR

Officially appointed by

THE AUTOMOBILE CLUB OF AMERICA Member Empire Tours Association

Oldest Hotel in America

Completely Remodeled

Albany to Poughkeepsie, N. Y., 75.6 Miles 0.0 From State St., Albany, south on Broadway.

0.5 Left across Hudson River bridge; at once right on Broadway, Rensselaer, 2 blocks.

1.2 Left on Columbia; keep left at Schodack Cen. 7.

8.8 Right at small garage; left 18.; right at Valatie 19.

24.1 Right fork Stuyvesant Falls; right at Stockport 27.5 right-left with trolley 33 . edge of Hudson.

33.2 Half left across Warren St.; left 32.

41.8 Left at end thru Blue Stores 45. and Rhinebeck 59 .

75.5 Left on Main to Market St., Poughkeepsie 75.6 .

\section{Smith Brothers Restaurant}

FaMOUS SINCE 1847

Owned and operated by the Makers of S. B. Cough Drops

Poughkeepsie, N. Y. 
New York to Kingston, N. Y., 96.6 Miles

0.0 From Dyckman St., New York, cross ferry, Hudson River. Upgrade into Englewood.

3.1 Half left, on Lafayette; left fork; $\mathbf{R}$ at end.

4.6 Left at end; curve $\mathrm{R}$ at 4-cors., Teaneck 5.3.

7.2 Left at 3-corners on Union St., Hackensack.

7.4 2nd right at church on Passaic St., left at water wheel, Arcola, 10.7; across bridge; then right twice.

14.5 Half right at 4-cors. on Maple St., Hohokus.

16.3 Left at hotel; thru Waldwick 17.; into Suffern.

24.0 Left at 3-cors.; thru Tuxedo $30.5 ; \mathrm{R}$ fk 32.6.

39.0 Right fork; thru Central Valley 47.

57.0 Right at Vails Gate; right on Broadway 61.2.

61.7 Left on Grand St., Newburgh; keep left 63.

63.1 Right fork; right at cross rd. 66.6 ; left 68.3 .

71.2 Left at Plattekill; next $R$ thru Modena 75.8.

81.3 Right at Normal Scho.; next L on Hasbrouck.

81.6 Right at end on Chestnut St., New Paltz.

94.6 Left at Wilbur; left on Greenkill; right on Fair to John St., Kingston 96.6.

\section{Kingston's Leading Hotel \\ THE EAGLE HOTEL}

Every room with private bath or running hot and cold water A cordial welcome to all

Special accommodations for Automobile Parties

New York Stock Exchange Office in Hotel

C. A. HUNGERFORD, Proprietor

\section{EAGLE GARAGE}

SERVICE STATION

Buick Hudson Cole Essex

PHONE 1083 KINGSTON, N. Y.

\section{Kingston to New York, 96.6 Miles}

0.0 From John St., Kingston, South on Fair St.; left on Greenkill; $\mathbf{R}$ under R.R. on Wilbur.

2.2 Right at end, Wilbur; bear left-right 3.2.

7.5 Right, thru Rifton 8.; to Main St., New Paltz.

15.0 Right on Hasbrouck; right-left at school.

25.2 Left at church; right at store Plattekill.

30.1 Left at 4-cors. into Young St.; $\mathbf{R}$ on Grand 34.

35.0 Right, B'way, Newburgh; L at church 35.5.

39.6 Left at Vails Gate; thru Central Valley 49.6.

80.1 Right at 3-cors., Suffern; into Hohokus, N.J.

82.1 Half left at 4-cors.; left fork; left at end.

85.8 Left across bridge; next right at Arcola.

89.2 Left on Union St., Hackensack; $\mathbf{R}$ at church.

91.3 Curve left at Teaneck; right at school 92.0.

92.7 Left on Lafayette; left fork; thru Englewood 94.

Down steep winding grade to ferry for Dyckman St., New York 96.6. 


\section{Kingston to Albany, N. Y., 58. Miles}

0.0 From Clinton St., Kingston, east on Albany;

0.2 Left fork; left fork at hotel 8.5 Glen Eyrie.

10.4 Left at 4-corners; right after Esopus Ck. bridge 11 .

11.4 Right on Main St., Saugerties; left at end 11.7 .

23.4 Left on Spring St., Catskill; thru Athens 28. 34.4 Half left at 4-cors., Coxsackie; $\mathrm{R}$ at inn 36.

45.0 Left at end; right across bridge; into Pearl St., direct to State St., Albany 58. miles.

\section{Albany to Kingston, N. Y., 58. Miles}

0.0 From State St:, Albany, south on Pearl.

2.2 Left fork after iron bridge at Kenwood.

23.5 Half right at 4-cors., Coxsackie; thru Athens 30.

34.5 Right at end on Bridge St., Catskill.

46.2 Right on Main St., Saugerties; left at hotel.

47.0 Left at end; right at 4-cors., 47.5; thru Glen Eyrie 50.; into Albany St. to Clinton St., Kingston 58.

\section{ARNINK GARAGE, Inc. STORAGE $\$ 1.00$}

Fire Proof - Most Central Location - Albany

SOUTH PEARL AND HUDSON AVE.

Troy to Albany Via Loudonville 11.3 Miles

C.0 Congress st., Troy, cross Hudson River, thru Watervliet .3, Lathams Corners 3, left thru Newtonville 4.3, thru Loudonville 5.4.

8.4 Four corners, $\mathrm{R}$ Viaduct, L 9.2, $\mathbf{R}$ 9.3.

10.0 Four corners, L Washington ave. 10.7, Educational Bldg. on left, State Capitol, right, left on State st., Pearl st., 11.3.

\section{Albany to Troy Via Loudonville 11.3 Miles}

0.0 From Pearl st., Albany, northwest on State, right-left at capitol, State Educational Building on right, .9 right on Northern Boulevard.

1.5 Left, right, Viaduct, 2.4 left thru Loudonville 4.5 .

5.8 Newtonville, 7.6 Lathams Corners, right thru Watervliet 10.8 over Hudson River to Troy 11.3 .

\section{HEALTH AND PLEASURE}

The people of this State now own nearly five hundred housand automobiles. And the work of investing in motor cars goes bravely on. More automobiles mean more time pent in healthful outdoor existence, more economy and :elerity in transportation, more good roads and more comfort and happiness generally. So more power to the horseless vehicle! 
Albany to Saratoga Springs, N. Y., 37.5 M.

0.0 From Pearl St., Albany, west on State; rightleft at Capitol 0.2 .

14.8 Right on Nott Terrace St.; left on Union 15.2 , next right on College St. to end.

15.7 Left on Nott St. under R.R. and next right.

16.5 Left across R.R. and Mohawk River.

16.9 Right at end, next left, over R.R. 17.2, joining route from Scotia, 18.1 .

22.2 Right after bridge; half $\mathbf{R}$ at Burnt Hills 23.3.

24.2 Left at end; keep right 26.5 into Church Ave.

31.2 Right on Saratoga Ave. Ballston Spa.

35.4 Left on Ballston Ave., left on Broadway 37.

37.5 Saratoga Springs.

\section{THE SARATOGA INN}

Best location on Broadway

Modern Appointments

Open all the year Steam Heat

Only European Plan in the village. Touring parties will find our Restaurant most excellent.

SARATOGA SPRINGS, N. Y.

Saratoga Springs to Albany, N. Y., 37.5 M.

0.0 From Saratoga Springs Broadway keeping right at Mon. 4.

1.7 Right at 5-cors. on Ballston Ave.

3.3 Right at cross road; right at end on Saratoga Ave. 34.0.

7.5 Left on Milton Ave., Ballston Spa.

14.5 Right; half left at Burnt Hills 42.1.

16.5 Left at end, across iron bridge.

20.6 Left fork; right at end 48.4; next left.

22.1 Cross Mohawk River and courve right on Maxon Rd.

23.0 Left at end, under R.R.; next $\mathbf{R}$ on College.

23.5 Left on Union; next right on Nott Terrace.

23.8 Left on State St., Schenectady.

37.3 Right and left at Capitol on State St.

37.5 Albany, State and Pearl Sts.

\section{THE NEW WORDEN}

\section{Broadway and Division St. - $\quad$ Saratoga Springs, N. Y.}

On main trunk line to the Adirondacks, in the center of New York State Mineral Springs Reservation. Accommodations for two hundred and fifty guests. Fifty rooms with private baths. Hot and cold running water. New Grill, Golf privileges to guests at new eighteen hole course.

American Plan $\$ 4.50$ per day and upward

Garage connected. Always open.

\section{E. C. SWEENY, Proprietor}

Drive slow, better be safe than sorry. 


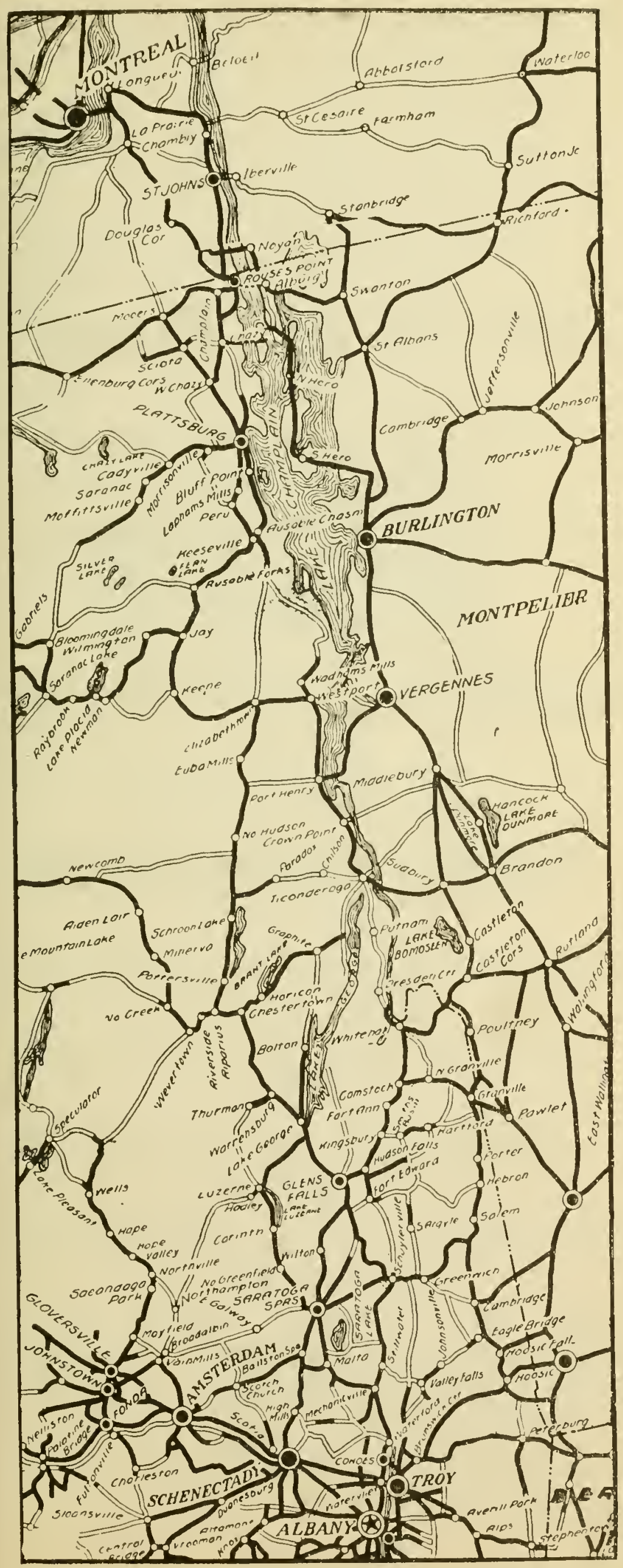


Saratoga Springs to Lake George, 27.9 Miles

0.0 Saratoga Springs Broadway right on Church St. at P.O.

0.1 Next left on Maple Ave.; through Wilton 7.4.

17.7 Half left on Main St.; cross Hudson River 55.7.

18.8 Keep left of Mon. Glens Falls.

27.9 Lake George-P.O. on left, north on main left fork 28.4 .

Lake George to West Port, N. Y. See page 18.

\section{TRockwell Tibouse}

Blens Jalls, 月. $\mathbb{1}$.

The best known commercial and tourists house in Northern New York. Re-decorated and refurnished throughout

RULIFF HOTEL EUROPEAN PLAN EDGEWOOD CLUB

Glens Falls Same management Alexandria Bay

Jreeman thotel Co., Inc., props.

B. W. FReEman, President

Bissell's Garage $\frac{\text { The Rockwell House Garage }}{\text { MOoN : MOTOR : CARS }}$

Supplies, Accessories, Repairing

GLENS FALLS, N. Y.

Fitzgerald's Restaurant and Grill

TOP OF GLEN STREET HILL

GLENS FALLS, N. Y.

The only first-class restaurant and grill in Glens Falls SEA FOODS A SPEGIALTY

Tourists Require no Bond upon entering Canada

See page 130

SKIDMORE TEA ROOM and Gift Shop

340 Broadway SARATOGA SPRINGS, N. Y. Telephone 1309-W

\section{Windsor Hotel $\substack{\text { ROUSES POINT, } \\ \text { NEW YORK }}^{-1}$}

Rooms with Bath Good Bass Fishing Garage Connections Best of Service and Food Open May 15 to Nov. 1 SEA FOODS A SPECIALTY

Tell them that you saw it in Motor Tours 
Saratoga Springs to Lake George via Lake Luzerne, N. Y., 32.6 Miles

0.0 From Broadway, Saratoga, northwest on Church st.

7.2 Left fork under R.R. thru South Corinth 10.3.

14.5 Half left at end, Corinth, following Hudson River.

19.8 Right into Hadley, and across river bridge.

20.3 Left around P. O., Luzerne.

20.4 Half right; left at end 20.6.

31.0 Left after underpass with trolley.

32.6 Lake George at P. O.

Ye Wayside Inn. Lake Luzerne, N.Y.

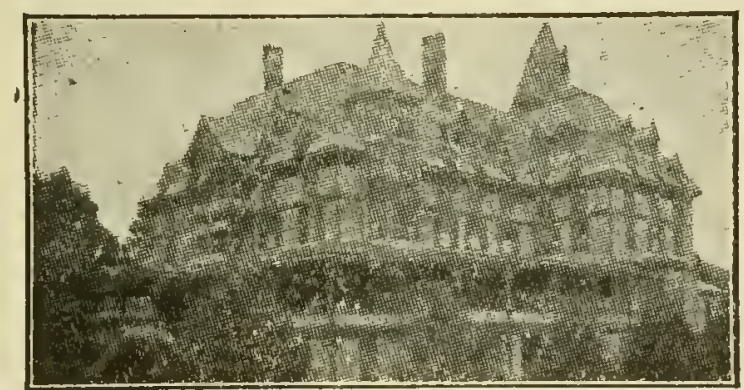

The Scenic Route "Saratoga to Lake George"

The House of Comfort for the tourist.

GEORGE W. LASALLE

Lake George to Saratoga Springs via Lake Luzerne, N. Y., 32.6 Miles

0.0 From P. O., Lake George, south on Main st. past Ft. Wm. Henry Hotel on left.

1.6 Right under R.R., on winding macadam thru woods.

11.8 Right; left at 12.0 .

12.2 Right around P. O. Luzerne, over Hudson River bridge and thru Hadley.

12.8 Left at prominent cross-road, following river.

18.1 Half right at monument Corinth; half left at S. Corinth 22.3.

25.4 Under R.R., at once R, coming into Church st.

32.1 Half right, still Church st. to Broadway.

32.6 Saratoga Springs at P. O.

\section{HOTEL MARION} ON LAKE GEORGE

(See next page)

\section{MARION GARAGE:- Opp HOTEL MARION \\ Telephone Lake Gaorge 127 F 5 \\ Garage in the Adirondacks}

We've made good because we make good. Gasoline, Oils and Accessories. First-Class Repair Shop. Direct New York City Service. Good Prices. Prompt Service. Efficient Methods. 
Lake George to Westport, N. Y., 51 Miles

0.0 From P. O. Lake George, north on Main st.

0.3 Right fork thru Bolton 8.1.

9.5 Right to Bolton Landing. Take boat for Sabbath Day Point. Bear right after landing past Silver Bay P. O. 11.9.

15.8 Half right over bridge at Hague into outskirts of Ticonderoga.

24.8 Left at water trough (straight on for town).

25.3 Right fork; left at end 32.8 .

33.5 $\mathrm{R}$ at cross st., Crown Point; $\mathrm{L}$ fork 34.1.

40.8 Keep right at Port Henry, and take next left 51.0 W'estport, Inn on right.

\section{ALGONQUIN HOTEL}

BOLTON ON LAKE GEORGE, N. Y.

American Plan, $\$ 4$ to $\$ 5$ per Day, $\$ 23$ to $\$ 30$ per Week

A Select, Comfortable Hotel for Discriminating People. Special

Attention to Motoring Parties. On Bolton_Parkway, 10 miles from Village of Lake George

Special Noon Dinner $\$ 1.50$

Under present management 23 seasons

E. G. PENFIELD, Prop.

\section{HOTEL MARION}

ON LAKE GEORGE, N. Y.

Most beautiful location on the Lake. Adjoins the new Country and Yacht Club. Golf and Tennis Tournaments. Large and vity rooms with private baths. All steamers

land in front of hotel. Fireproof garage. Write for illustrated booklet and rates.

\section{JOSEPH H. MARVEL}

\section{MARION GARAGE}

Telephone Lake George 127 F 5

Opp. HOTEL MARION

Largest one story Fire Proof Garage in the Adirondacks We're made good because we make good. Gasoline, Oils and Accessories. First Class Repair Shop. Direct New York City Service. Good Prices. Prompt Service. Efficient Methods EDMUND E. ALDRICH, Mgr.

\section{Diamond Point House} ON LAKE GEORGE

A Pleasant Place for a Short or Long Stop

Reasonable Rates

W. H. LA SALLE

The Westport Inn and Cottages WESTPORT, NEW YORK

On the shore of Lake Champlain, with all the amusements of a Lake and Mountain Resort. Most interesting, picturesque and well kept Golf Course on the Inn grounds with professional in charge. Select Clientele. 
0.0 Fron Inn, Westport, south on Main st. along lake shore.

10.2 Keep Y, at Port Henry 3-cors., R fork 10.5.

17.5 Left at cross street, Crown Point; right 18.2.

24.8 Right fork into outskirts of Ticonderoga.

26.2 Right at water trough (left leads to town).

35.2 Half left after bridge at Hague; past Silver Bay P. O. 39.

41.3 Sharp left to boat landing at Sabbath Day Point. Land at Bolton, and straight ahead.

41.5 Left at end, thru Bolton 43 and Marion 45.

51.0 Lake George at P. O. on Main st.

\section{NET FOUTE}

I NEW AND ATTRAC'TIVE OP'TIONAL ROUTE

\section{SAME MILEAGE FOR TOURISTS}

ound for Montreal, is to take the ferry at Westport, N. Z., for Thompson's Point, Vt. (14 miles south of Burlington), hence north thru Burlington and the islands of Lake Champlain o Alburgh, crossing the lake at Alburgh for Rouses Point, N. Y., n the new steamer "VENUS." Rates, car and driver \$1.25 ixtra Passengers 25c. each. The ferry from Westport, N. Y., to Chompson's Point, Vt., is on the steam yacht "ADMIRAL," ride of ten miles, affording magnificent views of Lake Chamlain and the Palisades of Split Rock Mountain. Rates on his boat, car and driver $\$ 1.50$. Extra passengers 25c. each Minimum on seven passenger cars and Limousines $\$ 2.00$

\section{B. J. WORNER}

Westport, N. Y.

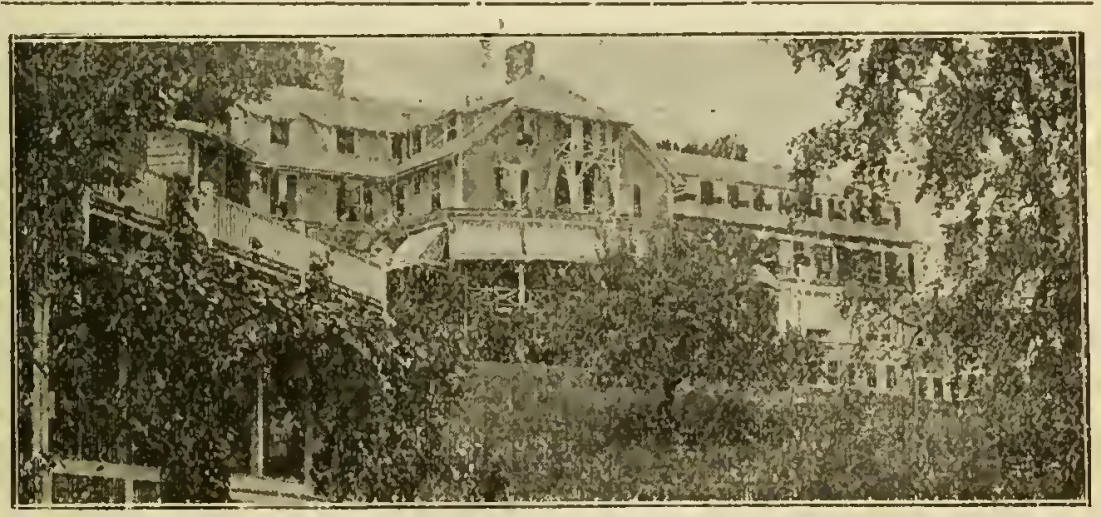

\section{The Westport Inn and Cottages}

\section{WESTPORT, NEW YORK}

$18 \mathrm{~N}$ the shore of Lake Champlain, with all the amusements of a Lake and Mountain Resort. Most interesting, picturesque and well kept Golf Course on the Inn grounds with professional in charge. Select Clientele.

Mrs. JAMES McKAY

Assistant Manager
H. O. SMITH

Manager 
Lake George to Elizabethtown, 68.5 Mile

0.0 Lake George P. O. on left, north on Mair

0.5 Left fork. Right to Westport page 18.

6.4 Right fork, Warrensburg; left at Chester town 8.3 .

22.5 Right at 3-cor.; right fork Pottersville 27.3 thru Schroon Lake 37.0; Schroon River 46.0

68.5 Left at hotels, Elizabethtown.

Elizabethtown to Malone, page 23

\section{ADIRONDACKS \\ DEERS HEAD INN and COTTAGES}

ELIZABETHTOWN, N. Y.

For people desiring the best. An American and European Hotel in the mountains. Best Golf Course in the Adirondacks.

\section{Benjamin F. Stetson}

\section{Prime Garage Elizabethown, n. y.}

Storage with Private Compartments Competent Mechanics

V. W. PRIME

Supplies

\section{BRENTWOOD INN}

ELIZABETHTOWN, N. Y.

On direct route from Albany to Plattsburgh and Montreal Special attention to tourists GARAGE IN CONNECTION

American plan $\$ 4.00$ per day and up

Phone 37

Elizabethtown to Lake George, 68.5 Miles

0.0 Elizabethtown at hotels, south thru Schroon Lake, 31.5.

41.1 Left at 3-cors., Pottersville; left fork 46.1.

50.1 Right at end, Chestertown; thru Warrensburg 64.1 .

68.5 Lake George at P. O., south on main road for Saratoga.

Lake George to Westport, N. Y. see page 18.

\section{GHESTER HOUSE}

Ghestertown, N. Y.

On great International Highway, halfway between Saratoga and Elizabethtown

Clean, Homelike, with good things to eat

Garage in connection

H. S. DOWNS, Prop. 
Elizabethtown to Plattsburgh, 54.9 Miles

0.0 Elizabethtown from hotels; left main road.

10.2 End of road take right.

12.2 Right at 3 -cors., Keene; left across bridge 18.5 .

27.8 Left at end. Ausable Forks; cross bridge right at hotel.

39.7 Cross Main St., Keeseville; left at end 41.4

41.7 Left at sign, Ausable Chasm, coming intc Charlotte St.

54.7 Left at end on Bridge St. to Margaret St. Plattsburg 54.9.

\section{The Famous}

\section{AUSABLE CHASM \\ and \\ HOTEL AUSABLE CHASM}

Open from June to October

Hotel modern in every respect and accommodates 400 . Private baths. Grill, music and dancing.

Special attention to automobile parties. Garage with all needed accessories.

THE CHASM

is the most impressive piece of natural scenery east of the Grand Canyon of Colorado

R. R. Station, P. O. and Telegraph address

AUSABLE CHASM, N. Y.

H. H. NYE, General Manager

\section{The WITHERILL}

Plattsburgh's Modern Hotel, located on Lake

Champlain. Open during entire year. Grill

Room. Garage. Service, and Rates Right

WM. H. HOWELL Plattsburgh, N. Y.

\section{TASKER GARAGE}

Next to the Witherall

\section{PLATTSBURG}

Road and Garage service day and night

HUNTERS' HOME ELIZABETHTOWN, N.Y.

Oniy hotel between Schroon Lake and Elizabethtown

Tourists entertained

Rates moderate

Directly on Great International Highway

$61 / 2$ miles south of Elizabethtown. 25 miles north of Schroon Lake

Thirty-third Season

J. S. LAVERTY, Prop. 


\section{Lake George to Saratoga, 27.9 Miles}

0.0 From P. O. Lake George, south on main road; keep left 1.4 .

9.1 Pass Mon. Glens Falls (on left) on Main st., across Hudson River Bridge.

10.2 Half right on Saratoga ave. with trolley thru Wilton 20.4 .

26.7 Half right on Maple ave. into Saratoga.

27.8 Right on Church st. Next left at P. O. on Broadway Saratoga Springs 27.9.

\section{Fitzgerald's Restaurant and Grill}

TOP OF GLEN STREET HILL GLENS FALLS, N. Y.

The only first-class restaurant and grill in Glens Falls SEA FOODS A SPEGIALTY

\section{HOTEL RUSSELL Corner Division and Glinton Streets.}

Rooms en suite with private bath. American or European Plan Also Granliden Hotel, Lake Sunapee, N. Y. \& Hotcl Weylin, Cor. Madison A ve and 54 th Street, New York City

J. THOMAS RUSSELL

CHARLES H. RUSSELL

\section{Plattsburg to Elizabethtown, 54.9 Miles}

0.0 From Margaret st., Plattsburg, E. on Bridge st.

0.2 Right on Charlotte; left fork at barracks 0.9 .

13.2 Left fork on Sable st., thru Keeseville 15.2.

26.9 Left at hotel, Ausable Forks across bridge; right 27.1 .

36.4 Left across bridge Upper Jay; at once right. 42.7 Left at 3-cor., Keene, left at end 44.7.

54.9 Elizabethtown end of road, at hotels.

\section{Huestis House and Annexes}

\section{SARATOGA SPRINGS, N. Y.}

American and European Plan

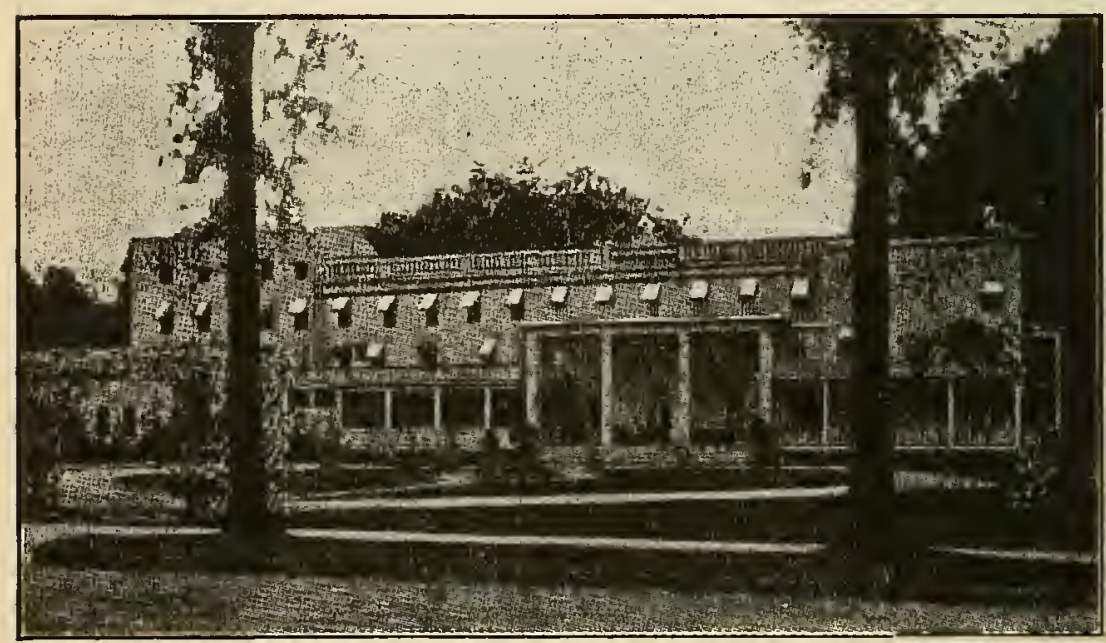

W. B. HUEST1S, Proprietor 
Elizabethtown to Malone, N. Y., 101.2 Miles

0.0 From Elizabethtown west; right 10.2; right at Keene 13.2; left across bridge; next right.

22.0 Left at Jay; left at Wilmington 27.2

40.4 Half left at Lake Placid; left 45.3; left at end. For Whiteface Inn turn right at 41.8.

50.0 Right at end; I on Broadway, Saranac Lake.

50.2 Right ar. hotel; left after 4-cors. Bloomingdale. Cross R.R. at Gabriels 62.2; right fork. At outskirts of Paul Smith's 65.8, thru McColloms 72.2 .

77.4 Left fork, Meachams; right at Duane 84.2.

85.8 Left; left at cemetery 96.7 into Duane St.

101.2 Main St., Malone, N. Y.

\section{Paul Smith's}

in the ADIRONDACKS

June to October

American and European Plan

Hotel-Casino-Cottages and Housekeeping Camp Life

On the St. Regis and Osgood Chain of Lakes

GOLF TENNIS BOATING EXCELLENT MOTORING

A la Carte Grill with Music and Dancing in the Hotel

ALL ROOMS WITH BATH

Telephone in every room. Steam Heat. Electric

Elevator. Through Daily Pullman Service

Paul Smith's Hotel Company

Paul Smith's - - - - New York

Che Owls Gead = Reene, Essex 60., D. Y.

Home Cooking and Comfortable Rooms

GARAGE STORAGE SUPPLIES MECHANICS

W. B. WASHBURN

\section{LAKE PLACID GARAGE \\ Phone 112}

Storage Supplies

Accessories

Expert Mechanics Taxi Service Towing Car FRED DASHNAW

Lake Placid, N. Y. 
Malone to Elizabethtown, 101.2 Miles

0.0 From Main St., Malone, south on Duane St.

8.4 Right at end; next left; right at cemetery

15.4 Right at end; left at end Duane 17.; left 23.

23.4 Right fork; thru McColloms 29.; Left at end 35.4 outskirts Paul Smiths; across R.R. Gabriels 39.

44.3 Right at end, Bloomingdale, to Saranac Lake.

51.0 Left on Broadway; right on Main; left on River.

53.8 Right on Flower Ave.; right at end 55.4. For Whiteface Inn left at 59.6.

60.8 Right at end, Lake Placid; $\mathrm{R}$ at Wilmington 74.

89.0 Left at Keene; left at end 91. to end of road. 101.2 Elizabethtown, hotel on right.

\section{WHITEFAGE INN ON LAKE PLACID ADIRONDACKS \\ NEW YORK \\ A modern hotel conducted on American plan, the only one located directly on the shore of Lake Placid, surrounded by miles of virgin forest and placid lake, owned in part by Adirondack Company and state. Superb mountain views. Two thousand feet above sea level. All suites arranged with bath. \\ Pure spring water supplied from our own reservoir located high upon mountain, hidden away from human habitation.}

\section{J. J. SWEENEY}

Winter Hotel, Highland Park, Aiken, S. C.

\section{THE BERKELEY}

Saranac Lake, N. Y.

American or European. Rooms en suite, rooms with baths. Homelike. Our meals will please you. Write for booklet.

OPEN YEAR ROUND

WALTER SAGENDORF, Prop.

Chestertown to Long Lake, N. Y., $55.3 \mathrm{M}$.

0.0 From Main st., Chestertown, run west.

4.1 Left fork; right at end 6.0.

9.6 Right at Wevertown; right at North Creek 14.9, thru Minerva 22, and Newcomb 41.

55.3 Long Lake.

\section{Wade's Restaurant}

NORTH GREEK, N. Y.

Popular Prices

First-Glass Food and Service

Rooms

Hot and Cold Water

Steam Heat

C. H. WADE 
Plattsburg to Montreal, P. Q., 72.3 Miles

0.0 From Margaret St., Plattsburg, W. on Cornelia.

0.6 Right at trolley on Montcalm; left at end 1.; right at end, 1.1.

1.3. Left at end; next $\mathrm{R} ; \mathrm{R}$ at Beekmantown 6.6.

10.4 Right at W. Chazy; left at end 11.1; thru Chazy 16.8.

19.9 Right at 4-cors.; left at end 21.; next right.

21.8 Half right at Coopersville into Rouses Point. Stop at U. S. Custom House and Register.

45.2 Left on Pratt St.; right at end on Church.

28.4 Left at Canadian Line; right at end 29.6. Stop at Canadian Custom House, Lacelle 34.8 for permit. No bond required,

45.2 Right at Douglass Cor.; left at St. Jacques 61.2 Left at end, on St. John's Rd.; right at La Prairie 63.2.

68.7 Left across Victoria Bridge; (toll 25c return) 70.8 Right on Wellington; left on McCord 71.

71.5 Right on Notre Dame; left on Cathedral St.

72.2 Left on Dorchester to Dominion Sq., Montreal 72.3 .

\section{Canada's Leading Hotel}

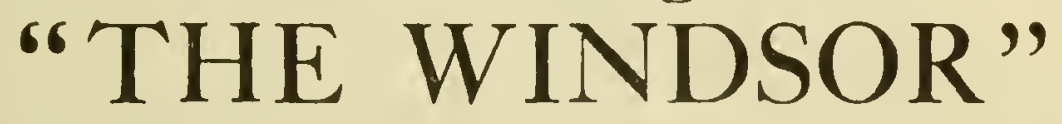

\section{Dominion Square}

MONTREAL - - - CANADA European Plan Exclusively

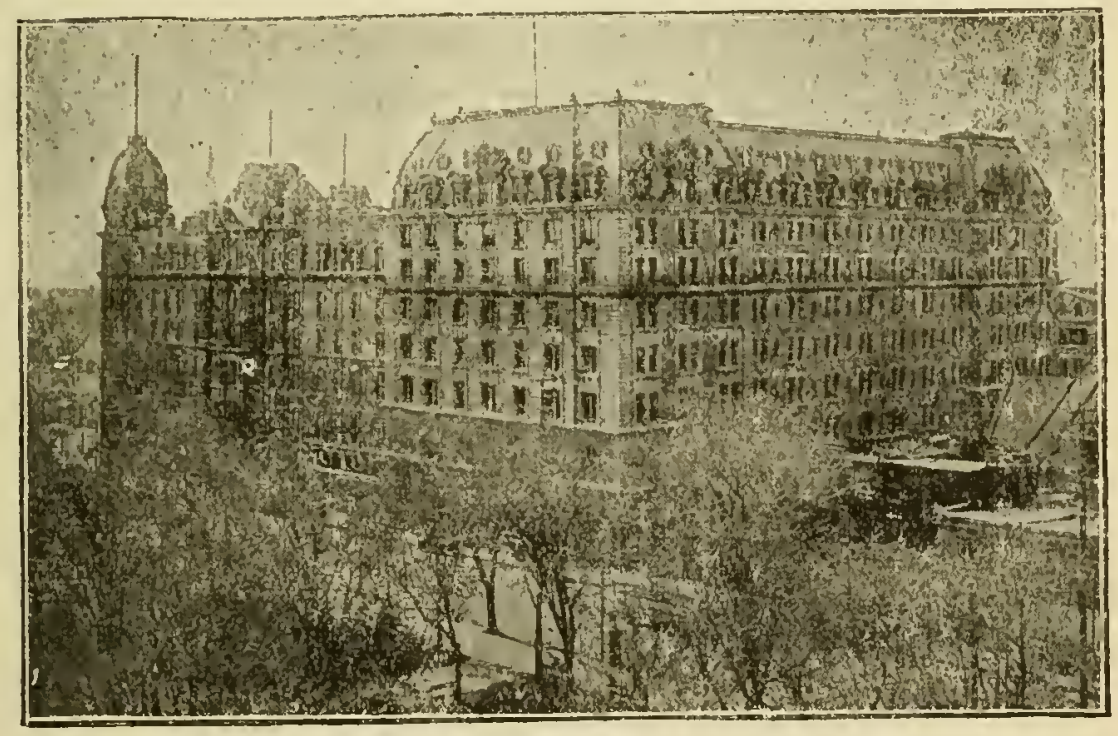

700 Rooms

450 with Baths

The location of the Windsor is unsurpassed for beauty and convenience. The ideal Hotel for Motor Tourists. Garage in connection.

JOHN DAVIDSON, Manager

\section{VICTORIA GARAGE}

REAR WINDSOR HOTEL 


\section{QUEENS HOTEL}

MONTREAL

Opposite Grand Trunk Stations

One block from Canadian Pacific

On main automobile route into city,

$\begin{array}{llllll}\text { American Plan } & - & - & - & - & \$ 5.00 u p \\ \text { European Plan } & - & - & - & - & 2.50 u p\end{array}$

MacNaughton Garage 650 Dorchester West

Montreal Quebec Canada

Near the

leading hotels

Storage for 250 cars on the ground floor

Montreal, P. Q., to Plattsburg, 72.3 Miles

0.0 From Dominion Sq., Montreal, east on Dorchester; next right on Cathedral; right on Notre Dame 0.5.

0.3 Left on McCord ; right on Wellington 1.2.

1.5 Left on Bridge St., across Victoria Bridge

3.6 Right at end; left at end, La Prairie 9.

11.1 Right across RR. on King Edward Highway ; right at end 21.4 .

27.1 Left at 4-cors.; thru Lacolle 37.5.

42.7 Left at U. S. boundary; right at end 43.9.

45.0 Left on Pratt St.; right at end, Rouses Pt.

50.6 Left at Coopersville, cross bridge : left 51.2.

51.4 Next R.; L. at 4-cors., 52.5; thru Chazy 55.5.

61.2 Right; left at 4-cors., W. Chazy 62.0.

65.7 Left at 4-cors., Beekmantown.

70.9 Left at end; next right; left 71.2.

71.4 Right on Montcalm St., left on Cornelia 71.7.

72.3 Plattsburgh, Cornelia St. at Margaret.

\section{HANNAN \& HENRY GARAGE}

Most up-to-date and best stocked garage in Northern New York

Distributors for

Cadillac - Studebaker - Chevrolet - Mack Trucks Goodyear - United States Tires

ALWAYS OPEN 
Albany to Amsterdam, N. Y., 31.0 Miles

0.0 From Pearl st., Albany, northwest on State, right-left at Capitol direct to Schenectady.

15.1 Right at end on Washington; left beyond bridge.

16.0 Half right with trolley at Scotia; keep lefi with trolley.

31.0 Market and Main sts., Amsterdam.

\section{BARNES HOTEL AMSTERDAM'S \\ BARNES HOTEL LEADING HOTE.}

\section{ftlodern likstaurant}

J. J. KENNEDY - $\quad$ - $\quad$ - $\quad$ Proprietc

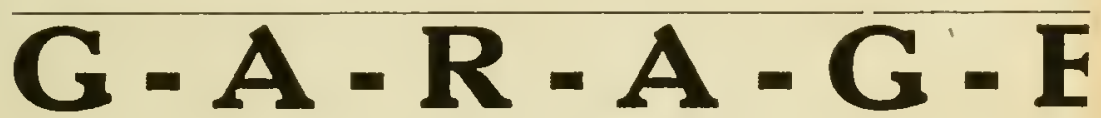

BIRGH \& JOHNSON

Rear Barnes Hotel

Night and Day Service

Competent Mechanics
Amsterda1

Storage and Supplies

Telephọe 6

\section{HOTEL W ARNER}

Under new and experienced management. Largest as most quiet hotel in the City, with cool rooms for touris Modern-European plan. Fire-proof garage.

101-103 East Main Street

AMSTERDAM

Amsterdam to Albany, N. Y., 31.0 Miles

0.0 From Market st., Amsterdam, east on Mai thru Scotia 15.

15.5 Right across Mohawk River; 3d left St: st.

16.4 Right fork at park, Schenectady, on Alba st., to Robinsor ; left-right on State st. dir into Central ave.; jog right-left at Capi to Pearl St., Albany 31.0.

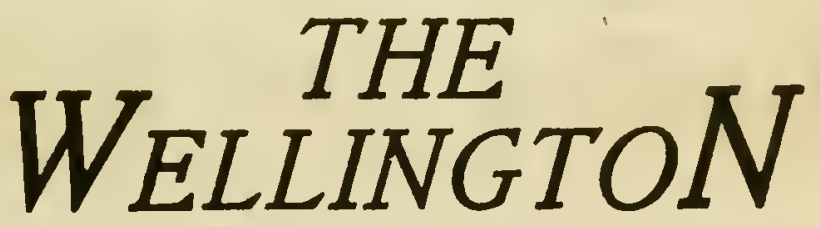

MODERN

FIRE-PROOF

HOME-LI] AIRY ROOMS OVERLOOKING THE HUDSI

Courteous Treatment to All

Single

$\$ 1.75$ to $\$ 3.00$

with bath

$\$ 2.50$ to $\$ 4.00$

136 STATE ST.
RATES

Double $\$ 2.50$ to $\$ 4.00$ with bath $\$ 4.00$ to $\$ 6.0$ 
Amsterdam to Utica, N. Y., 63.8 Miles

0.0 From Market st., Amsterdam, west on Main.

6.8 Left fork, Tribes Hill; left fork, Fonda 10.4; thru Nelliston 24.7; St. Johnsville 30.7; Little Falls 41, into Albany st., Herkimer.

48.1 Right at end on Main; left at 4-corners 48.5.

52.2 Left fork; left at end, Deerfield, cross bridges on Genesee to Bleecker st., Utica 63.8

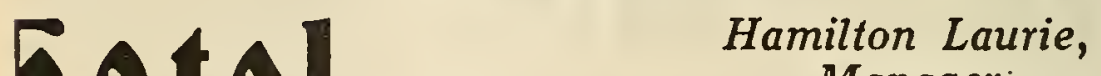 Manager. \\ totel \\ ITTLE FALLS, N. $Y$.

0 outside rooms, mostly with bath. Every window frames a :ture of the wonderful Scenic Mohawk Valley. A strictly first ss hotel furnishing excellent food at a reasonable price. Halfy between New York and Buffalo, halfway between Albany d Syracuse.

\section{BRONNERS GARAGE}

7 West Lansing, Near Richmond Hotel LITTLE FALLS, N. Y.

Jome of Overland, Willys-Knight, Hudson, Essex Cars and Republic Trucks

Prest-O-Light Service Expert Mechanics

Utica to Amsterdam, N. Y., 63.8 Miles ).0 From Bleecker st., Utica, north on Genesee. 1.4 Right at Deerfield; left fork 14.8 on Church. ;.3 Right on Main st., Herkimer; left at Sta. 15.7, on Albany st.; thru Little Falls 22.8; St. Johnsville 33.1; Nelliston 39.1 ; Fonda 41.3.

:2 Right fork on Main st., Amsterdam 63.8; left fork optional.

\section{HOTEL CONRAD}

\section{AMSTERDAM, N. Y.}

ictly Modern Fireproof European Plan

Rates: Without Bath, $\$ 1.50$ up

With Bath, $\$ 2.00$ up

\section{SIDNEY GARAGE}

IN REAR 


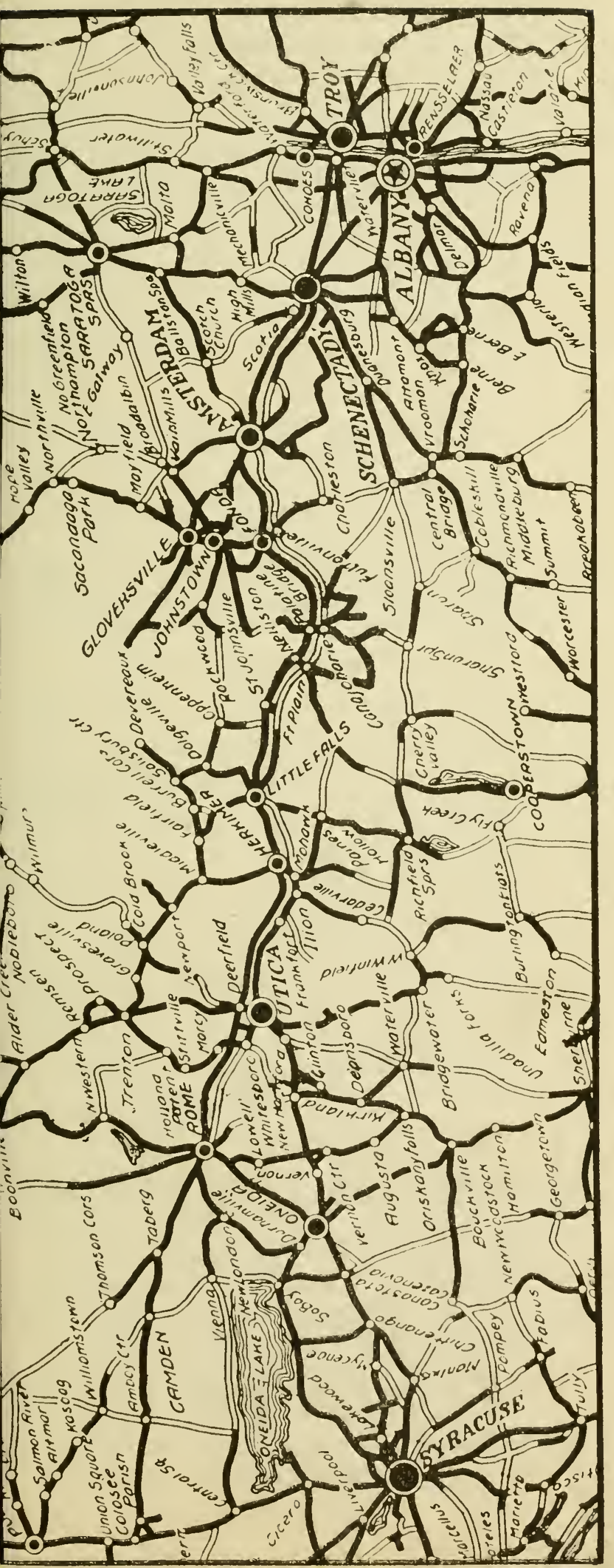


Fonda to Lake Pleasant, $\mathbf{N}$. $\mathbf{Y} ., 53$ Miles

0.0 From station, Fonda, start north.

3.9 Left at trolley on William st., Johnstown.

4.3 Right on Main st.; next left on Market.

4.5 Left on Washington st.; left at end; next $\mathbf{R}$

6.4 Half R; R at sign 10.9; thru Newkirk 16.

18.3 Left at end; half right at Caroga Lake 18.6 Pass Green Lake P. O. 21.6 thru Arietta 28

35.8 Left fork; thru Spy Lake 41.8, and along Lake Piseco.

44.5 Right at end Rudeston; left fork at Lake Pleasant $\mathrm{P}$. O.

53.0 Speculator P. O. at N. end of Lake Pleasant

\section{Hotel Morley}

Located in the Adirondacks at Lake

Pleasant, Hamilton County, New York

(1) NE of the few new hotels built for the comfort of tourists.

Appointments strictly modern. Magnificent view at an elevation of 2000 feet. Music, Dancing, Fishing, Tennis, Boating, Bathing. Open May 28th to October 1st.

Rates, American Plan, \$6 up

R. P. CORNELL : : : Manager

Isake Pleasant to Fonda, N. Y., 53 Miles

0.0 From P. O. Speculator, southwest along Lake Pleasant.

8.5 Half left at Rudeston; thru Spy Lake 11.2; and Arietta 25.

34.4 Half left at Caroga Lake Village.

34.7 Right; thru Newkirk 37; left at end 42.1.

48.3 Left on Mill st., next right on Washington st.

48.5 Right on Market st. ; second right on Main st., Johnstown.

48.7 Left on William st.; right on Madison; left on Chestnut 53.0 Fonda, at station.

\section{Hotel Kingsboro}

Only Fireproof Hotel in Fulton County

Re-Decorated and Newly Furnished

Highest Standard of Service with Reasonable Prices

European Plan Garage Connected

Under New Management with Hotel Oneonta of Oneonta. N. Y., and Pontiac of Oswego, N. Y.

H. M. STANFORD, Prop.

E. G. STANFORD, Mgr.

GLOVERSVILLE, N. Y. 
Amsterdam to Lake Pleasant, N. Y., 60.3 M.

0.0 From Main st., Amsterdam, north on Market, thru Perth 6.0.

7.4 Left fork; left at Vails Mills 8.5.

Bear right Broadalbin 9.5.

15.5 Right at sign into Harrison st.

16.8 Right on S. Main st., Gloversville, 17.5.

18.7 Right at water trough on Eighth ave.

18.9 Left at end on Kingsboro ave.

19.3 Right fork from trolley; right at end.

20.9 Left at end; right at Raceville 23.4.

23.7 Left; right at end thru Mayfield 24.

27.2 Right at school ; L fork Cranberry Creek 29.

32.7 Left at cross road; right 33.3 ; left 33.5 thru Sacandaga Park.

34.4 Right over bridge; $\mathrm{L}$ at end, Northville 34.9.

35.0 Half $\mathrm{L}$ along river, thru Hope 42 ; Wells 51 .

60.3 Speculator, north side of Lake Pleasant.

Gateway to the Adirondacks

KENNYETTO INN

Broadalbin, Fulton Co. New York

Turn Right at Vails Mllls

Healthiest spot in the state Rates reasonable

Send for booklet

Lake Pleasant to Amsterdam, N. Y., 60.3 M.

0.0 From Speculator S. E. along Lake Pleasant, thru Wells 9.3, Hope 18.3; L fork 24.2.

25.3 Half right at end, next left over bridge at Northville.

25.9 Left thru Sacandaga Park.

26.8 Right; left 27.0 ; right 27.6 at cross road.

31.2 Right fork over bridge, Cranberry Creek.

33.1 Right at school past Mayfield P. O. 36.

36.5 Left; right at end 36.7; left at Raceville 36.9.

39.4 Right on State st.; left on Kingsboro ave. 41.

41.4 Right on Eighth Ave.; left on Main st., Gloversville 41.6.

43.5 Left on Harrison st.; left at end 44.8.

51.8 Right at Vail's Mills; thru Perth 54.3.

Left at Vail's Mills to Broadalbin 52.8.

60.3 Market and Main sts.. Amsterdam, N. Y.

\section{BARNES HOTEL \\ AMSTERDAM'S LEADING HOTEL MODERN RESTAURANT}

J. J. KENNEDY, Proprietor

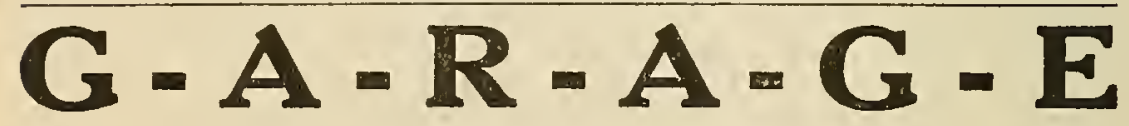

BIRCH \& JOHNSON

Rear Barnes Hotel

Night and Day Service

Competent Mechanics
Amsterdam

Storage and Supplies

Telephone 647 
Utica to Syracuse, N. Y., 50.4 Miles

0.0 From Bleecker St., Utica, S. W. on Genesee St.

3.6 Half right with trolley at New Hartford; right fork 6.5 .

8.3 Left at end across R.R. thru Kirkland; Vernon 16.7 .

19.7 Left under R.R.; at once R, under R.R. 20.2.

20.8 Over R.R. bridge, at once right; thru Oneida Castle 32.

33.6 Left at 5-cors., Chittenango; right fork 34.3.

37.7 Right at store, Mycenae; L 38.6 along canal.

41.8 Right across canal; left fork 42.

45.4 Right over R.R. bridge; at once left, trolley. 47.8 Left fork on James st.; bear $\mathrm{L}$ into Warren st. 50.4 Jefferson St., Syracuse 50.4.

\section{(II) If \\ "THE INN DEAUTIFUL"}

Syracuse, N. $Y$.

Fireproof Central Convenient

The best accommodations for tourists

Club Breakfasts 60c Luncheons 60c Dinner 85c

Rates: $\$ 1.50, \$ 1.75$. With bath $\$ 2.00$ and up.

Opposite Public Library

L. G. ABBOTT, Mgr.

BISSELL GARAGE On the Level

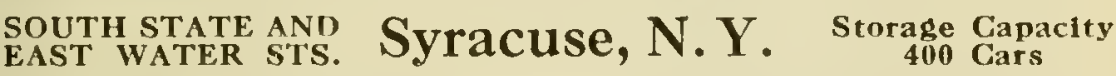

\section{HOTEL ST. CLOUD}

\section{SYRAGUSE}

Corner Fayette and Clinton Street

"Tourists Home"

Syracuse to Utica, N. Y., 50.4 Miles

0.0 From Jefferson St., Syracuse, north on Warren $\mathrm{St}$.

0.2 Half right on James with trolley; keep L 3.5.

3.9 Half right at sign; $R$ over R.R. then left 4.9.

8.6 Left at end; right at end 11.8 ; left at end; Mycenae 12.7 ; curve left thru Chittenango 16.

16.8 Right at 5-cors.; thru Oneida Castle 28.7.

29.6 Left at mon. over R.R. bridge; under R.R. 30.1 .

30.7 Left at end under R.R.; right curve at factory; thru Vernon 33.6; Kirkland 39.9.

41.1 Right after R.R.; half $L$ at New Hartford 47.4.

50.4 Genesee and Bleecker Sts., Utica. 
Syracuse to Rochester, N. Y., 97.6 Miles

0.0 From Mon. Syracuse, W. on Genesee, under R.R. at Camillus 8.1 ; thru Elbridge 15.4

20.6 Left at 4-cors., Sennett; half left on Fulton 25 ; next right on Genesee St., Auburn.

25.6 Right on State; next left on Clark St.

32.2 Right at 4-cors.; curve left-right 32.8 .

37.5 Left at sign; right at trolley on Falls St., Seneca Falls.

44.0 Left on Main St., Waterloo; over canal and R.R. ; right on River St. 44.4.

48.9 Sharp right over R.R. bridge ; left after R.R. 51.4 on Exchange St.

51.7 Right on Seneca St., Geneva; left at end 51.9 on Main.

52.0 Right at park; right at Church 52.1.

53.3 Left at 4-cors.; next right; under R.R. at Flint 58.4.

67.0 Half right at end of Lake St. on Main, Canandaigua.

74.9 Left at end; $\mathbf{R}$ over R.R. bridge, then $\mathrm{L} 77.6$.

79.6 Left across 2 R.R.'s, then right; right to Mendon 83.6; thru Pittsford 90.3 into East Ave.; half left on Main 97.4.

97.6 Rochester, Main St. and Clinton Ave.
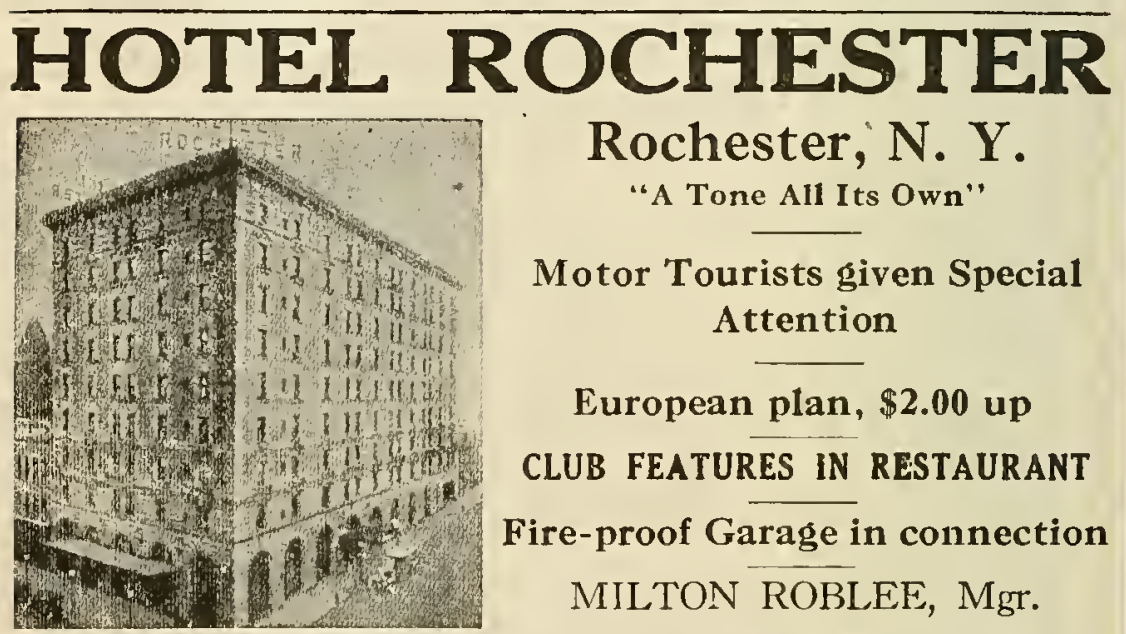

Rochester; N. Y.

"A Tone All Its Own"

Motor Tourists given Special Attention

European plan, $\$ 2.00$ up

CLUB FEATURES IN RESTAURANT

Fire-proof Garage in connection

MILTON ROBLEE, Mgr.

\section{ROCHESTER GARAGE "On the Level"}

Connection Hotel Rochester Largest Fire Proof Garage in the City

Supplies-Accessories First-class mechanics Service car 26 SOUTH PLYMOUTH AVENUE, ROCHESTER

Rochester to Syracuse, N. Y., 97.6 Miles 0.0 From Clinton Ave., Rochester, E. on Main St.

0.2 Half right on East Ave.; thru Pittsford 7.3.

8.4 Left fork; left at Mendon 14.; right at end 18.; thru Victor 19.5 ; right over R.R. bridge at once left 20.2 .

22.7 Right at yellow house; into Main St., Canandaigua.

30.6 Half $L$ on Lake St.; under R.R. at Flint 39.2.

44.2 Left at 4-cors.; next right on Washington St. 
45.1 Right at end; curve left on Main St.

45.7 Right at end around park; left on Main St.

45.9 Next right on Seneca; left at end on Exchange St., Geneva.

46.2 Right on Lake St.; sharp left after R.R. bridge 48.7 .

53.2 Left at 4-cors. into Waterloo; right with trolley 54 .

57.1 Left on Cayuga St., Seneca Falls; half R 57.7.

60.1 Right at end; left at 4-corners; $R$ fork 70.8 .

71.8 Right at end; left at end on Genesee St., Auburn.

72.4 Left on Fulton; next half right on Grant St.

77.0 Right at 4-cors., Sennett; left fork 77.7 thru Elbridge 82.2 ; under R.R. at Camillus 89.5 into Genesee St.

97.6 Syracuse at Monument.

\section{HOTEL ST. CLOUD}

\section{SYRACUSE}

Corner Fayette and Clinton Street

"Tourists Home"

BISSELL GARAG ti On the Level SOUTII STATE AND
EAST WATER S'TS. Syracuse, N. Y.

Rochester to Buffalo, N. Y., 76.3 Miles

0.0 From Clinton Ave., Rochester, West on Main St.

0.4 Left at hotel on Plymouth; $\mathrm{R}$ on Brooks 3.4.

2.5 Left at store; half right at 4-cors. 3.0.

5.1 Left fork; right at Scottsville 12.6; left 13.

19.1 Left at Mumford; right at mon. Caledonia 20.5; thru Leroy 27.3; Stafford 31.7; Batavia 37.7; Pembroke 50.6; Clarence 58.6; Williamsville 66.6 into Main St., to monument, Buffalo, N. Y. 76.3 .

\section{Wootel : TRicbmono}

\section{BATAVIA, N. Y.}

Leading Hotel midway between Rochester and Buffalo. Re-opened under new management July 15 th, 1920, 65 rooms, hot and cold running water in every room. Baths, European Plan. Rates $\$ 2.00$ up. Special rates by the month. Club Breakfasts, Business Men's Lunch served daily in the Coffee Shoppe. Dining room service $6 \mathrm{~A}$. M. to $12 \mathrm{P}$. M. MUSIC DANGING

Garage and Repair Shops in Rear 
Buffalo to Rochester, N. Y., 76.3 Miles

0.0 From Monument Buffalo, north on Main St. with trolley. Keep right 5.5 thru Williamsville 9.7 ; Clarence 17.7 ; Pembroke 25.7 ; and E. Pembroke 32.2 .

33.6 Left fork at C. H., Batavia; keep right 39.9 thru Leroy 49.

55.8 Left at mon. Caledonia; $\mathrm{R}$ at Mumford 57.2.

63.7 Left at end, Scottsville; half $L$ at 4-cors. 73.5.

73.8 Right at store on Brooks, left at end 73.9.

75.9 Right on Main St., Rochester to Clinton Ave. 76.3 .

RICHMOND GARAGE

Auto Storage rear Hotel Richmond

BATAVIA, NEW YORK

AUTOS and TIRES Repaired Experienced Mechanics

Rear HOTEL RICHMOND

Prices reasonable

Phone 61-M

H. C. PARSONS, Manager

STOP AT THE WHITCOMB HOUSE

ROCHESTER, N. Y.

Garage in connection

Rochester's Popular Priced European Hotel

Rates - - - $\$ 1.50$ up -with bath $\$ 2.50$ up

Cor. East Main St. and Clinton Ave., South

Erie, Pa., to Buffalo, N. Y., 92 Miles

0.0 From Park, Erie, northeast on Sixth street.

1.2 Left-right across East ave.; left fork 3.5.

6.5 Right; left at end, with trol, turn N. E. 15.2.

18.7 Sharp left at end, curve right over bridge; thru Ripley 22.7; danger, 'right across R.R. 26.2 ; thru Westfield 30.4 ; Brocton 38.7 ; Fredonia 45.4 and Sheridan 51.3.

57.0 Right from trolley at park, Silver Creek.

57.3 Left with Central ave.; L-right-L, Irving, with concrete across bridge and under R.R.

60.8 Right at end; right at school, Farnum 62.6.

66.6 Left at stores, Brant; half left 68.7.

70.5 Half left; right at end; next left 71.5.

71.6 Right at store, Evans Cen.; right fork Louisiana at 90.8 .

90.8 Right fork after drawbridge on Louisiana st.

91.6 Left on Elk st. to Main st., Buffalo, 92.0.

Full Course Luncheons $50 \mathrm{c}$.

11 A. M. to 2.30 P. M.

\section{T H E}

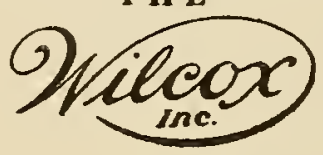

Full Course Dinners

90c. $-\$ 1.00-\$ 1.25$

5 P. M. to 9 P. M

Restaurant - A la Carte Service at all hours

Music 12 to $2 \mathrm{P} . \mathrm{M}$.
287-289 WASHINGTON ST. (near Swan) Music BUFFALO, N. Y. 6 to 8 P. M. 


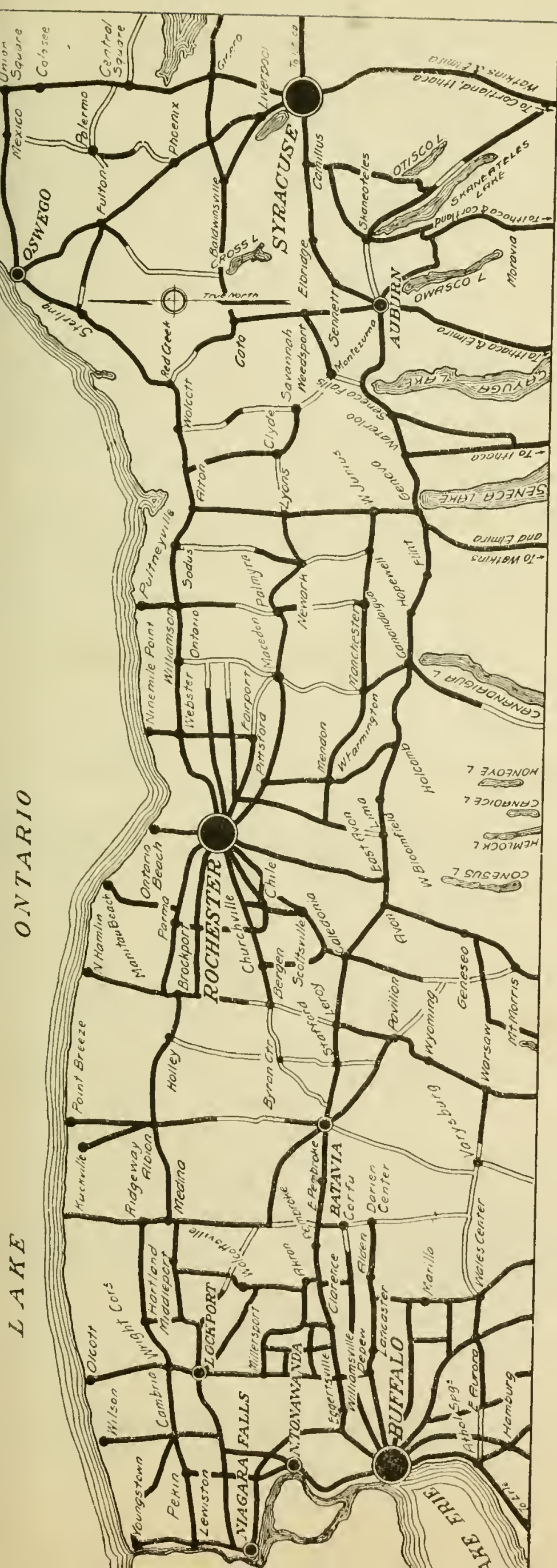


Syracuse to Buffalo, N. Y., 154.5 Miles

0.0 From mon., Syracuse, west on Genesee, unc R.R. at Camillus 8.1 ; thru Elbridge 15.4 .

20.6 Left at 4-cors., Sennett; half left on Fult st. 25, next right on Genesee st., Auburn.

25.6 $\mathrm{R}$ on State st.; next $\mathrm{L}$ on Clark st. (Trolle)

32.2 Right at 4-cors; curve left-right 32.8 .

37.5 Left at sign; right at trolley on Falls Seneca Falls.

44.0 Left on Main st., Waterloo; cross canal a R. R. ; right on River st., 44.4.

48.9 Sharp right over R.R.; left after R. R. 5 on Exchange st.

51.7 Right on Seneca st., Geneva; L on Main 5

52.2 Right on Hamilton St. direct into Lake

67.0 Half right at end on Main st., Canandaig

67.8 Left after R.R. on West st.; thru E. Bloo field 75.7 and West Bloomfield 81.1; Li 85.1; Avon 93 .

99.0 Half left at mon., Caledonia; thru Leroy 1

116.0. Bear right with trolley at C. H. Batavia t Pembroke 128.7 and Williamsville 144.8 i

154.5 Buffalo, Main st., at monument.

\section{Osborne Hotel}

AUBURN, N. Y.

LYNN G. SWIFT, Prop.

150 Rooms, 80 with Bath Excellent $G$

Garage in Connection

LEONARDS GARAGE

Next to the Osborn House Auburn, N. Y.

Open day and night

Franklin - Marmon - Studebaker - Millburn Ele

BOYCE GARAGE

Seneca Falls, N. Y.

The heart of the Finger Lake section

Chevrolet Sales and Service

E. J. FARRINGTON, Manager

THE GOULD

European

Rates $\$ 1.50$ and upwards

Club breakfasts
100 rooms. 75 with b. Table d'hote lunche

L. J. STAUFF

Seneca Falls, N. Y. 
Buffalo to Syracuse, N. Y., 154.5 Miles

0.0 From monument Buffalo, north on Main st., with trolley thru Williamsville 9.7 and Pembroke 25.7 .

38.5 Left fork at C. H., Batavia; trolley leaves 39.8.

55.5 Half right at monument, Caledonia; thru Avon 62.5; Lima 69.4; W. Bloomfield 73.4; E. Bloomfield 79.

86.7 Right at trolley on Main st., Canandaigua.

87.5 Half left on Lake st.; under R. R. at Flint 96.

02.5 Left at end of Hamilton St. on Main St., Geneva.

02.8 Next right Seneca st.; left at end on Exchange.

03.1 Right on Lake st. ; sharp left after R.R. 105.6.

10.1 Left on Main st., Waterloo; right with trolley 111.

14.0 Left on Cayuga st., Seneca Falls; half right 114.6.

17.0 Right at end; left at cross road 121.4 ; right fork from trol. 127.7.

28.7 Right at end; next left on Genesee st., Auburn.

29.3 Left on Fulton; next half right on Grant.

33.9 Right at stores, Sennett; left fork 134.3 ; thru Elbridge 139.

46.3 Right under R.R. at Camillus into Genesee st.

54.5 Syracuse, Genesee st., at monument.

\section{THE WEBSTER HOTEL}

Grill and Coffee Shop always open

Modern Home Cooking

Away from the trains

CANANDAIGUA, N. Y.

\section{THE MARKS GARAGE}

8-38 Phoenix Street

Largest Garage in the City

Canandaigua, N. Y.

We Never Close

Expert Repair Men at All Times

Ires and Accessories

Battery Service Station

\section{The NEW JEFFERSON}

S. Salina and W. Jefferson SYRACUSE, N. Y.

KRESS and SEMBACH, Props

I modern, high class hotel. Under lew management. Extensive alterations. Just completed.

$1.50 \mathrm{Up}$

Garage

In the Heart of the City

Hotal near to N.Y.C. Station D.L.W. Station Shoppin: District Amusement Past Office

TELEPHONE IN ALL ROOMS

BISSELL GARAGE On the Level iOUTH STATE AND
iAST WATER STS. Syracuse, N. Y. 
Erie, Pa., to Cleveland, O., 101 Miles

0.0 From the park, Erie, south on State st.

0.1 Right on Eighth st. with trolley; left fork 6.1

15.0 Left; left at end across R.R. on Church st 16.6.

17.1 Right at end, past monument, Girard 17.3 thru E. Springfield 23 and W. Springfielc 26.5 .

30.3 Curve right-left over viaduct, Conneaut 0 thru Amboy 34.4 and North Kingsville 38.5

43.3 Left at East Village; right with trol. 44.

44.6 Left on Main st., Ashtabula, with trolley.

44.7 Next right on Center st.; curve left on Prospect thru Geneva 54.8.

57.8 Left at cross-road; right at Unionville 59.

72.4 Right-left at park, Painesville, with trolley thru Mentor 78.3.

82.8 Left at bank, Willoughby on Erie st. coming into Euclid ave. to

101.0 Public Square, Cleveland, Ohio.

Cleveland, O., to Erie, Pa., 101 Miles

0.0 From Public Sq., Cleveland, east on Euclio ave.

18.2 Right from trolley on Glenn ave., Willoughby.

28.6 Right-left at park, Painesville, with trol.

42.0 Left at Unionville; right at cross-rd. 43.2.

46.2 Thru Geneva, follow trolley into Center st.

56.3. Left on Main, next right Spring st., Ashtabula; left at end with trolley.

56.7 Right with trolley at East Village thru Amboy 66.7 .

70.5 Cross viaduct at Conneaut, curving right-left beyond.

81.5 Right fork from trolley into Girard.

83.9 Left on Church st., from trolley; right after R.R. into 8th st.

100.9 Left on State st. to park, Erie, 101.

KEEP A RECORD OF GASOLINE

In Keeping any record, it is obvious Some rules must govern procedure. The above are submitted as necessary.

It Will Be Understood that the Full Tank is necessary to start and continue the record. The Full Tank positively determines the Amount of Consumption for Mileage Obtained. It is not always possible to determine this otherwise.

Explanation: Assume that your brand of gasoline is used; that the record is started January 10th; that the motor tank holds, Full, twenty gallons-

Fill the tank with whatever amount is necessary to do so and enter 20 against January roth. Also the Odometer Reading which is assumed to be 3520 .

January I2th it is necessary to refill and 12 gallons is required to do this. Enter 12 against January 12th, also Odometer Reading which is shown to be 3850 . By deducting the reading of January roth (3520) from that of January I2th (3700) I 80 miles is determined as the mileage on the I2 gallons consumed, or 15 miles to the gallon, as Shown by the amount Just Pumped.

Keep a record monthly to show condition of your motor. 


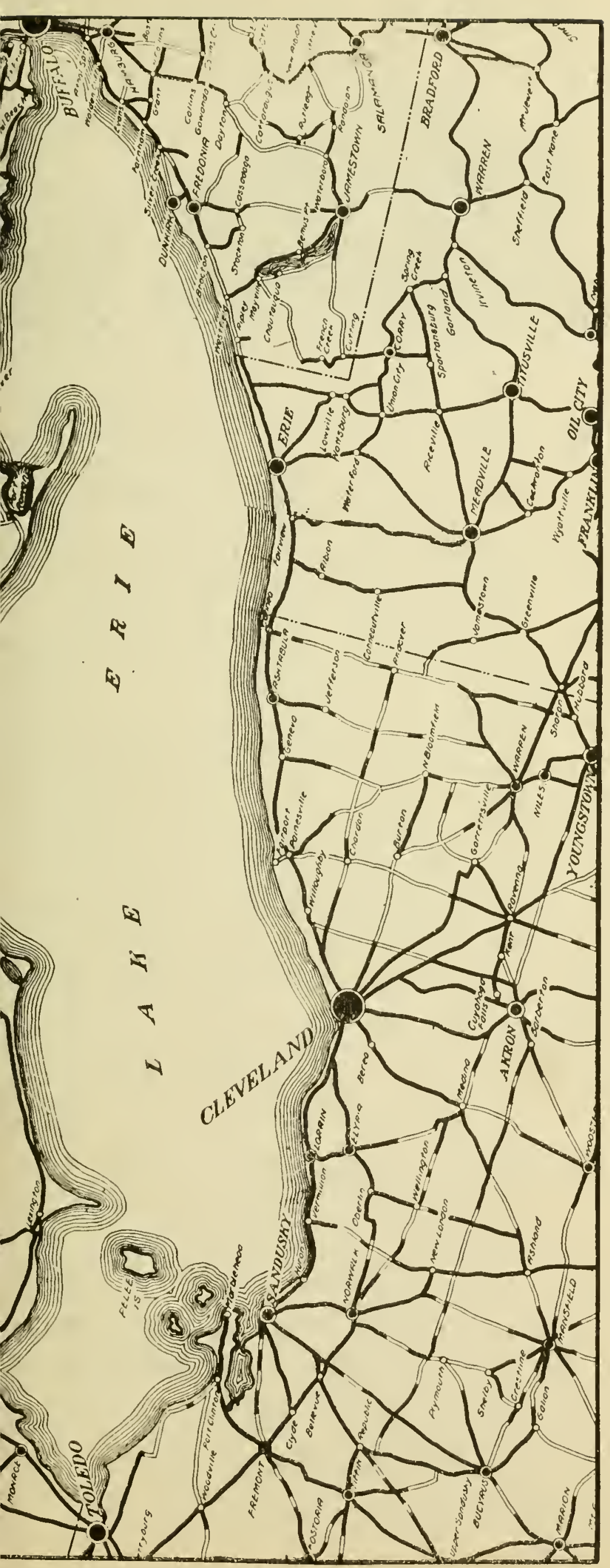




\section{HOTEL MARKEEN}

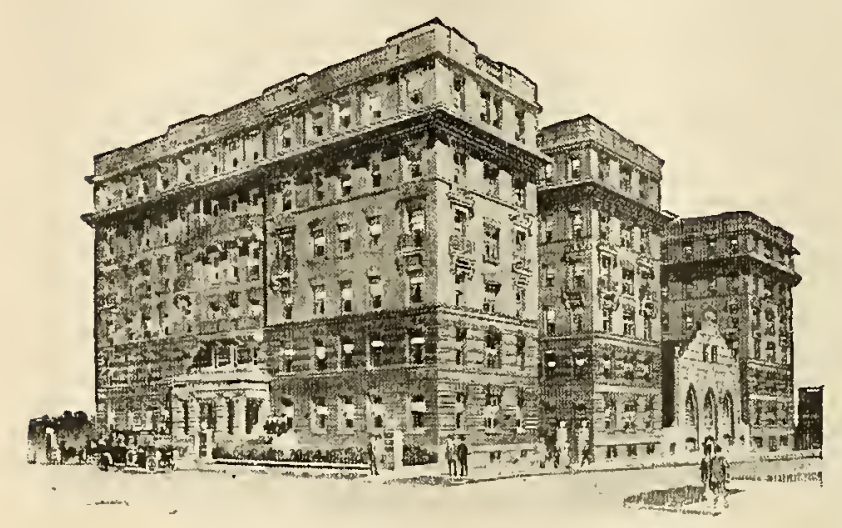

Absolutely

Fireproc

Our Own Fire proof Garage

Located on

Premises

\section{Main and Utica Streets}

\section{BUFFALO - - - NEW YORK}

\section{Buffalo, N. Y., to Erie, Pa., 92 Miles}

0.0 From Main st., Buffalo, southeast on Elk st

0.7 Right on Louisiana st. with cross-trolley.

20.4 Left with concrete; next right; half left 20.7 25.4 Right at Brant; left at end, Farnum.

31.2 Left under R.R.; right-left-right at Irving

34.7 Right down hill with Central ave., Silve Creek.

35.0 Left at trolley on Main st., thru Sheridan 40.7 Fredonia 46.6; Brocton 53.3; Westfield 61.6

65.8 Danger, right across R.R.; thru Ripley 69.3

73.3 Sharp right after bridge; thru Northeast 76.8

84.3 Right from trolley at sign; left at end.

90.2 Left-right across East ave., with trolley.

92.0 Erie, at park, 6th and State streets.

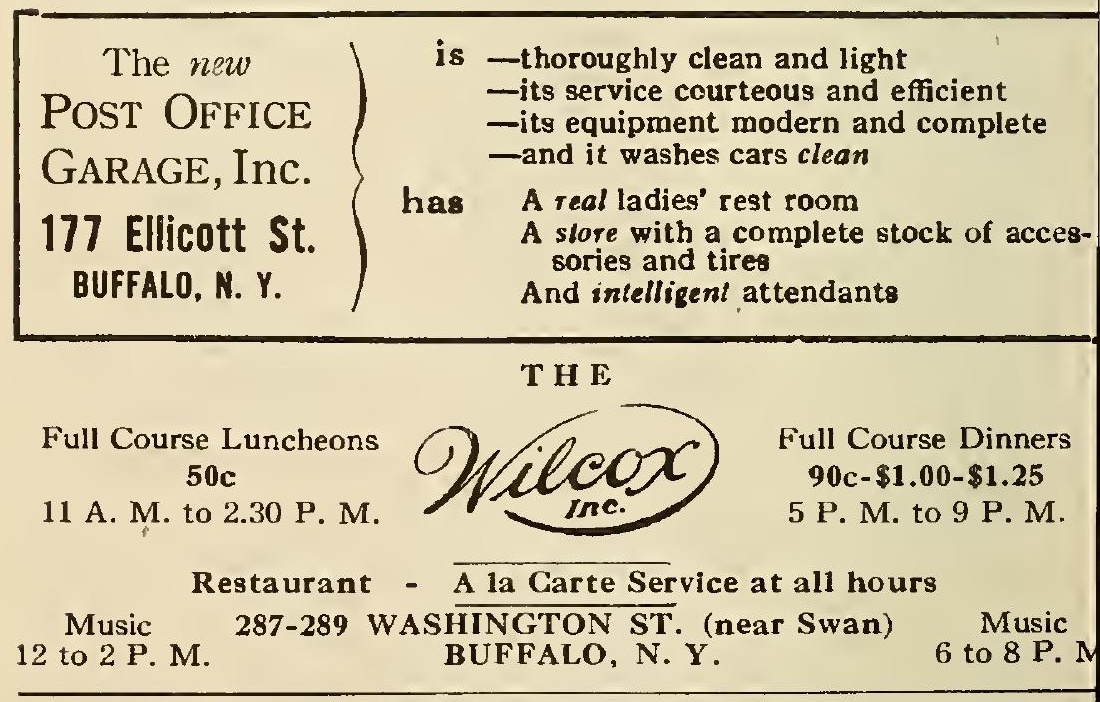

Scranton to Wilkes-Barre, Pa., 18 Miles

0.0 From Wyoming ave., Scranton, northwest on Lacka wanna.

0.3 Half right over bridge, Lackawanna river.

0.6 Left with trolley; curve right, Jackson st.

1.0 Left at bank, Main st.; thru Holland 5.3.

6.3 Right after bridge; thru Pittston 10.3.

10.9 Right fork into River st., Scranton.

17.8 Left on Market to Public Square 18. 
Buffalo to Olean, N. Y., 71.4 Miles

10 From Main st., Buffalo, east on Swan st., thru Ebenezer 8; into Main st., East Aurora.

2 Right on Olean st.: thru Holland 26.7.

7 Left fork; thru Chaffee 33.3 ; Yorkshire 36 ; Delevan 39.

12 Left at hotel, Machias; right at end on Main st. 487; thru Franklinville 51 .

Left fork, Ischua; thru Hinsdale 64.6; into Main st. Left with trolley on Union st. to P. O. Olean 71.4.

Olean to Buffalo, N. Y., 71.4 Miles

.0 From P. O, Olean, north on Union st.

0.8 Right at end with trolley thru Hinsdale 6.8.

9 Left fork across RR; thru Ischua 12.9; and Franklinville 21 .

2.6 Left fork; right at hotel, Machias 27.2; thru Delevan 32.4: Yorkshire 35.4, and Chaffee 38.

3.8 Left fork; left fork after bridge 41 ; thru Holland 44.7, and South Wales 50.

4.2 Left on Main st. East Aurora; right fork 55.2; thru Ebenezer 63.4 ; into Seneca st., into Swan st.

1.4 Buffalo, Main st.

\section{T H E}

11 Course Luncheons $50 \mathrm{c}$.

A. M. to 2.30 P. M.

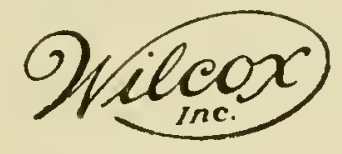

Full Course Dinners 90c. $-\$ 1.00-\$ 1.25$

5 P. M. to 9 P. M.

Restaurant - A la Carte Service at all hours

Music 287-289 WASHINGTON ST. (near Swan) Music to 2 P. M.

BUFFALO, N. Y.

6 to 8 P. M.

OTEL MARK.EEN ABSOLUTELY FIREPROOF

Our own fireproof garage on premises

ain and Utica Street

Buffalo, N. Y.

Olean, N. Y., to Dubois, Pa., 94.5 Miles

From Union st., Olean, east on State.

6.4 Right over bridge; right over bridge 7 .

Left at end; thru Eldred 13.5; Coryville 19.

Left at end; into Main st., Smethport 26.4.

Left on Marvin st.: thru Hazelhurst 37.

Left fork; thru Wilcox 49; right 52.2.

Left after railroad on Main st., Ridgeway, past Court

House.

2.3 Right fork; half right 65.9; thru Crenshaw 78.

9.5 Right; left at end 79.6 ; left after station, Brockwayville 80.3 .

0.3 Left at cross road; thru Falls Creek 91.

13.9 Right across railroad on boulevard to postoffice Dubois 94.5 .

Dubois, Pa., to Olean, N. Y., 94.5 Miles

0.0 From postoffice Dubois, north on Brady st.

0.6 Left at end; left fork 0.8; thru Falls Creek 39.

4.2 Right at cross road; right at end after railroad 9.9.

4.2 Right across railroad; right at end Brockwayville.

4.9 Right; left at end 15; thru Crenshaw 16.5.

32.3 Half left on Main st., Ridgeway; right across railroad at station 32.8 .

38.1 Right fork; left over bridge; thru Wilcox 40.5 .

37.7 Left on Marvin st.; right, Main st., Smethport.

58.7 Left fork; right fork 72; thru Coryville 76 ; Larabee 78 , and Eldred 81 .

33.2 Right after bridge; left after bridge 87.5.

38.1 Left after bridge; thru Portville 89 into

94.5 State st., Olean, at Union. 
New York to Binghamton, N. Y., 190.5 M.

0.0 From Dyckman St., New York, cross fe Hudson River. Land, wind upgrade Englewood.

3.1 Half left on Lafayette; left fork; $R$ at end

4.6 Left at end; curve $R$ at 4-cors., Teaneck

7.2 Left at 3-corners on Union St., Hackens:

7.4 2nd right at church on Passaic St., left water wheel, Arcola 10.7 ; across bridge; $t$ right twice.

14.5 Half right at 4-cors. on Maple St., Hohol

16.3 Left at hotel; thru Waldwick; Suffern 2 Tuxedo 50.5 ; Southfield 34.6.

39.1 Left fork; thru Harriman 39.2; Monroe

47.6 Right at 3-cors.; right at trough, Chester

52.3 Half left on Main St., Goshen; right f

58.4 Right at 3-cors.; $L$ with trolley 61.4 on $M$

61.8 Left on Mill St., Middletown; left at end 6.

73.2 $\mathrm{R}$ fork at P. O., Cuddebackville; next le

80.5 Right at Hartwood Sta.; half L 84.3; L

89.3 Left at C. H. Monticello; right at mon. $t$

Liberty 91.4.

107.4 Right; left fork 108.6; right 111.; to Roso

118.0 Left at church; L at end thru Cooks Falls 1

134.3 Left; left at end; thru Hancock 146.5.

147.0 Right; ṇext left at end, thru Hales Eddy 1

159.7 Left on 2nd St., Deposit; right at end 16

173.1 Right; $\mathrm{R}$ beyond bridge 175 , into Windsor

175.5 Left after bridge; right along river 185.8 in

190.5 Court St., Binghamton at Court House.

\section{THE GOSHEN INN}

GOSHEN, ORANGE COUNTY, N. Y.

An English Inn of the Elizabethan Period

OPEN MAY 22nd TO OCTOBER 15th

Service a la Carte

Open Air Dining Terrace Overlooking the Historic Goshen Race Track. Exceptionally large and Splendidly Appointed Rooms with Private Bath.

CHAS. HUERSTEL, Manager

\section{HOTEL BENNETT}

Under new management. Completely reconstructed and furnished. Modern in every respect. Refined service. Our table is unexcelled. When you come to Binghamton this summer stop at the BENNETT you will find it different

BINGHAMTON, N. Y.

\section{JUNE AUTOMOBILE COMPANY}

\section{Storage for Hotel Bennett}

BINGHAMTON, N. Y.

PEERLESS, DORT and GRANT CARS 
Elmira to Watkins, N. Y., 23 Miles

0.0 From Water st., Elmira; north on Baldwin; half right on Union; left on Division; right on Central 2.

6.2 Right at end; next left, Horseheads; right at end under R. R. at once left 12.3 .

19.7 Left at end, Montour Falls; right at end.

23.0 Glenn ave. at Fourth st., Watkins.

\section{HOTEL JEFFERSON}

At Watkins' Glen on Seneca Lake

Open all the Year

WATKINS, N. Y.

Watkins to Elmira, N. Y., 23 Miles

0.0 From Fourth st., Watkins, S. on Franklin st.

0.7 Left cross R. R.; right Owego st., Montour Falls 3.3.

16.6 Right at Horseheads, next left Central ave.

21.2 Left on Division st.; right Lake st.

22.4 Right fork, Baldwin st. to

23.0 Water st., Elmira.

\section{Clinton House}

Heart of Finger Lakes District ITHACA, N. Y.

Under New Management

European Plan

Watkins to Ithaca, N. Y., 27.8 Miles

0.0 From Fourth st., Watkins, S. on Franklin st.

2.5 Left; right beyond bridge; left fork 3.

9.6 Left at end; right at Alpine 10.3 ; left 10.4.

11.5 Left at end; half left 11.7 ; right 16.1 ; left 19.

23.6 Left at end; into Cayuga st.

27.8 Ithaca, State and Cayuga sts.

When in ITHACA, N. Y.

"Heart of the Finger Lakes"

STOP A'T

\section{Lang's Palace Garage}

Storage Rates $50 \mathrm{c}$, and $75 \mathrm{c}$.

High Class Service at Reasonable Rates

\section{Ithaca to Watkins. N. Y.. 27.8 Miles}

0.0 From State st., Ithaca, south on Cayuga st., bear right on Spencer 0.3.

4.2 Right; left at cem. 8.8; left 11.8 .

16.4 Right after bridge; left at Alpine; next right 117.4; thru Odessa 21.6 ; to Montour Falls.

25.3 Left at end; right at end on Franklin st., Watkins; to Fourth st. 27.8. 
Olean to Westfield, N. Y., 81.6 Miles

U.0 From Main St., Olean, west on State; right at end, Alleghany 4; left at 4-cors. 4.2; thru Vandalia 9.

17.8 Right after R.R.; Salamanca; L. on Main St.

18.7 Right on Broad; $\mathbf{R}$. at end; $\mathbf{L}$. over bridge.

25.2 Right at cross rd.; over bridge; next left.

34.9 Left; left at cross rd. 36.8; right fork Randolph 37.2; jog right-left 53.5, into 3rd St., Jamestown.

54.0 Right on Washington Ave.; left 60.5 to Bemus Pt.

63.9 Right at end; thru Pt. Chautauqua 71.; and Mayville 75.

81.6 Portage St. at Main Westfield.

\section{THE SAMUELS}

Absolutely Fireproof

European Plan

The last word in COMFORT, LUXURY and SERVICF.

JAMESTOWN, N. Y.

Located on Main Trunk Lines of Western New York

QUICK LUNCH ROOM ANI GRILL ROOM ATTACHED

Westfield to Olean, N. Y., 81.6 Miles

0.0 From Main St., Westfield, S. E. on Portage St.

6.6 Left at C. H. Mayville; right fork, Hartford 8.6 ; thru Point Chautauqua 10.6 ; to Bemus Point.

17.8 Left at hotel; right at end 21.1 ; right 26.4 .

27.6 Left on 3rd St., Jamestown; jog right-left 28.1 ; thru Falconer 30.5; Randolph 44.4; right 44.8

55.9 Right over bridge; left at cross rd.

61.1 Right at trolley on Center; left on Broad.

62.9 Left on Main St., Salamanca; R. on Wildwood.

63.6 Left across R.R.; thru Vandalia 72.6.

77.4 Right; next left at trolley thru Alleghany 78. ; into State St. to Main St. Olean 81.6.

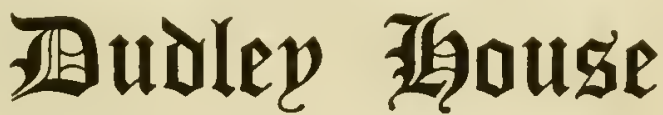

SALAMANCA, N. Y.

American Plan.

Rates, $\$ 3.50$ up.

J. LANSING MOORE, Mgr.

COLUMBIAN INN

Modern summer hotel, all conveniences OPEN MAY TO NOVEMBER

Fishing, Golf, Dancing, Tennis Rates: \$4.00 per day American

Chicken and Fish Dinners a Specialty

GEO. RAPPOLE, Mgr. 
Binghamton to New York, N. Y., 190.5 Miles

0.0 From C. H. Binghamton N. E. on Court St. with trolley.

4.9 Left at end; $\mathbf{R}$ across bridge into Windsor 15.4 .

15.6 Left across Susquehanna river; L at end 17.3.

26.7 Left at McClurs; left at edge of Deposit on 2nd St. 29.8.

30.6 Right on Pine St.; thru Hales Eddy 36.; right-left 43.3, thru Hancock 44. ; right across bridge, next right.

Continued on page 52

Binghamton to Elmira, N. Y., 59 Miles

0.0 From Washington st., Binghamton, west on Main st.

8.3 Left at Union, across river; right at Vestal 9.

21.9 Right across bridge into Owego; right-left 22.2 .

22.3 Left on Main st.; left at end 23.6; right 25.5.

27.8 Left at Tioga P. O., thru Smithboro 33; and Barton 35.

36.5 Left at end; into Chemung st.; thru Waverly 41.3 .

57.7 Left at end on Water st. to Main st., Elmira 59.
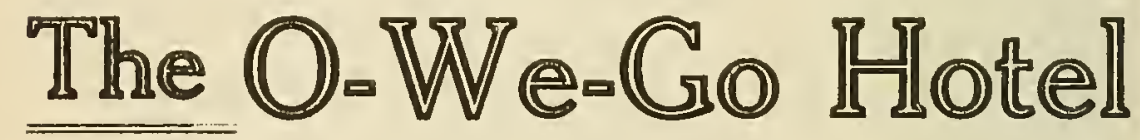

OWEGO, N. Y.

Under New Management

Unexcelled Dining Room

Table d'Hote and A La Carte Service

Operated to make it a hotel for discriminating guests

OWEG0 GARAGE STORAGE REARRNG
60-62 CENTRAL AVE. OWEGO, N. Y.

\section{HOTEL RATHBUN \\ Centrally Located}

Modern, American and European plan

G. H. \& S. F. De VED

Elmira, N. Y.

LA FRANCE GARAGE

Modern Fire Proof Garage

Near all hotels. Capacity 150 cars

ELMIRA, N. Y. 


\title{
Olean to Elmira, N. Y., 126.4 Miles
}

0.0 From P. O., Olean, north on Main; R-L 0.7.

6.8 Left at Hinsdale; right 7.5; right at end 11.8.

14.3 Right at end Genesee st.; left at Cuba 14.5.

17.5 Right under R.R.; right at Belvidere 17.7.

30.2 Half left at Belmont; $R$ at sta. to Wellsville.

39.9 Left on Dike st.; left at end 41.4; right 41.8 .

48.6 Half left at 5-cors.; left 56.6; next $\mathrm{R}$ Alfred.

60.0 Right at end; right across bridge 63.1.

65.4 Right on Main st., Hornell ; right on Canisteo st., 66.

72.5. Left over bridge; right at end; thru Adrian 76.4 ; Cameron 84.4; Addison 87.4.

106.2 Right at Painted Post; right on Bridge st., Corning, to Market st. 108.4; Big Flats 116.4.

123.9 Right at Elmira Heights coming into Main street.

126.4 Water St., Elmira.

\section{HOTEL FASSET}

\author{
E. F. GEE
}

On Liberty Highway

WELLSVILLE, NEW YORK

American Plan. Rooms with or without Private Bath

\section{Elmira to Olean, N. Y., 126.4 Miles}

0.0 Right on Main st.; left fork on College Pl. along R.R.

2.5 Left at end on Central ave., Elmira Hgts.

4.9 Left under R.R.; left over R.R. bridge; right 8.6; thru Big Flats 10.0 .

18.0 From Market st., Corning, north on Bridge.

18.6 Left on Poultney; left at Painted Post 20.2; thru Addison 29; Cameron 42; Canisteo 45.

60.5 Left on Main st., Hornell; left 61 ; left 53.2.

69.6 Left fork, Alfred; next right; left-right 78.

84.6 Left over bridge; right 85 ; right on Main st. 96.3 ; thru Wellsville.

95.7 Left at sta., Belmont; half right at park.

98.5 Left at Belvidere; left at end; thru Friendship 93.6.

102.0 Right on Genesee st., Cuba; left after R.R.

125.8 Left on Main st. to P. O., Olean 126.4.

\section{DONT'S FOR MOTOR DRIVERS}

Don't attempt to cut in between street cars going in opposite directions.

Stop ten feet behind cars taking on or discharging passengers.

Don't forget that a street car cannot turn out.

Don't forget that slow moving vehicles should drive close to the curb. 
Little Falls to Cooperstown, N. Y.. 34.6 Mi.

0.0 From Main st., Little Falls, south on Ann st., across Mohawk River and R.R.

0.4 Right at end along R.R.; left at 3-corners, upgrade 2.2 .

7.8 Right at end, next left, Paine's Hollow. '

9.9 Right fork with macadam; left at Jordanville 12.7 .

17.8 Left at park on Main st., Richfield Springs.

23.7 Sharp right; right at P. O., Springfield Center 24.4.

34.5 Right on Chestnut st., to Main st., Cooperstown 34.6.

Cooperstown to Watkins, N. Y., 127.2 Miles

0.0 From Lake St., Cooperstown, south on Chestnut.

2.2 Right; left at Fly Creek 5.7; thru Burlington 14 .

16.3 Left at end; thru Edmeston 20.3; and Sherburne 34 .

38.4 Right fork, Smyrna; left fork 41 ; right 41.1.

47.4 Left at end, Otselio; right 48.7 ; thru DeRuyter 58.

66.2 Left at Truxton; R. at end 76.6; to Cortland.

77.3 Left on Main; L.-R. on Tompkins, at 5-cors.

87.4 Right at Dryden P.O. ; half R. 94.5; R. 95.6.

98.4 Left on University Ave.; R. on Mill St. 99.

99.2 Left on Cayuga St., Ithaca; across State St. 99.4.

103.6 Right; left at cem.; 108.2; left 11.2.

115.8 Right after bridge; left at Alpine; next right 117.4; thru Odessa 121.; to Montour Falls.

124.7 Left at end; right at end on Franklin St., Watkins; to 4th St. 127.2.

Watkins to Cooperstown, N. Y., 127.2 Miles

0.0 From 4th St., Watkins, S. on Franklin St.

2.5 Left; right beyond bridge; left fork 3.0.

9.6 Left at end; right at Alpine 10.3 left 10.4 .

11.5 Left at end; half left 11.7 ; right 16.1 ; left 19.

23.6 Left at end; into Cayuga St., Ithaca 27.3.

28.0 Right on Mill St.; left on University Ave.

31.6 Left at end; left at Dryden P.O. to Cortland.

49.7 Left on Main St.; half $\mathbf{R}$. on Clinton; $\mathbf{R}$. fork 51.

61.0 Right at Truxton; thru DeRuyter 69.2; $\mathbf{R}$. 71.7 .

79.9 Right; right at Otselic 79.8; thru Smyrna 89.; and Sherburne 83.; follow main road thru Edmeston 107.

111.0 Right; thru Burlington 113.2, half right, Fly Creek 121.5.

125.0 Left at end on Chestnut to Lake St., Cooperstown 127.2. 
0 From Front st., Kingston, 110rth on Waslington st.

2 Right at 3-corners; thru West Hurley 6.6.

1) Right at 3-corners, Boiceville; left at Mt. Pleasant 21; thru Phoenicia 24.7; Allaben 29.

Left at church, Shandaken; right fork 36.

Left at 3-corners, Arkville; right 46.4.

Right at end, Margaretville; thru Roxbury 60.

Left at square, Grand Gorge, to Stamford.

Half left, Harper st. ; thru Harpersfield 77.

Left fork thru covered bridge; thru Davenport 86.7.

Right fork over Susquehanna river into

8 Main st. at Broad st., Oneonta.

\section{at at---KILKENNY'S

Oneonta to Kingston, N. Y., 99.8 Miles

.0 From Broad st., Oneonta, southwest on Main st.

6 Left fork uphill; thru Davenport 13.1.

Half right Main st., Stamford.

Right at Grand Gorge; thru Roxbury 39.8.

Left at 3-corners, Margaretville; right fork 55.

Half left, Pine Hill; bear right into Shandaken 69.2; thru Phoenicia 75 ; bear right 78 .

Left at Boiceville; thru Ashokan 86.8.

6 Left at 3 -corners; into Washington st. to

8 Front st., Kingston.

operstown to Little Falls. N. Y., 34.6 Mi.

0.0 From Main st., Cooperstown, north on Chestnut.

0.1 Left on Lake st., follow shore of lake.

0.2 Left at Springfield Center P. O.; left on Cherry Valley Turnpike 10.9.

5.8 Right on Church st., at park, Richfield Springs.

9.3 Half left at Cullen; right at end Jordansville 21.9 .

2.5 Left fork; right-left at Paine's Hollow 26.8.

2.4 Right at end along R.R.; left across tracks and bridge over Hudson River 34.2.

4.6 Little Falls, Main and Ann sts.

\section{SAFETY DON'TS FOR MOTORISTS}

Don't forget to slow down at street or road intersections. may save a human life.

Don't forget to look to the right and the left before ssing a railroad crossing.

Don't forget, when signaled by a vehicle in your rear, turn slightly to the right and allow such vehicle to pass 4 on your left.

Don't forget the pedestrians. They, too, have rights on streets and highways.

Don't forget that all motor vehicles must be equipped with equate brakes in good working order and adequate horns other signaling devices.

Don't forget that in case of accident, you must stop imdiately, and give your name, address and license number the injured party or a police officer. If no police officer in the vicinity or the place of injury or accident, report - same to the nearest police station or judicial officer. 
Geneva to Watkins, N. Y., 42.6 Miles

0.0 From Exchange st., Geneva, west on Senec

0.2 Left on Main st.; right on Hamilton 0.5 .

2.0 Left at 4-corners; next right.

3.9 Left; right 6.1 ; left 6.5 .

8.2 Half left, Hall's Cors.; thru Penn Yan 17

18.4 Half right; left at cross $r d .25 .4$; right $2 t$

29.5 Right thru Dundee; left 34 ; left-right

38.1 Left beyond Reading Cen.; right at end.

42.6 Glenn ave., at Fourth st., Watkins.

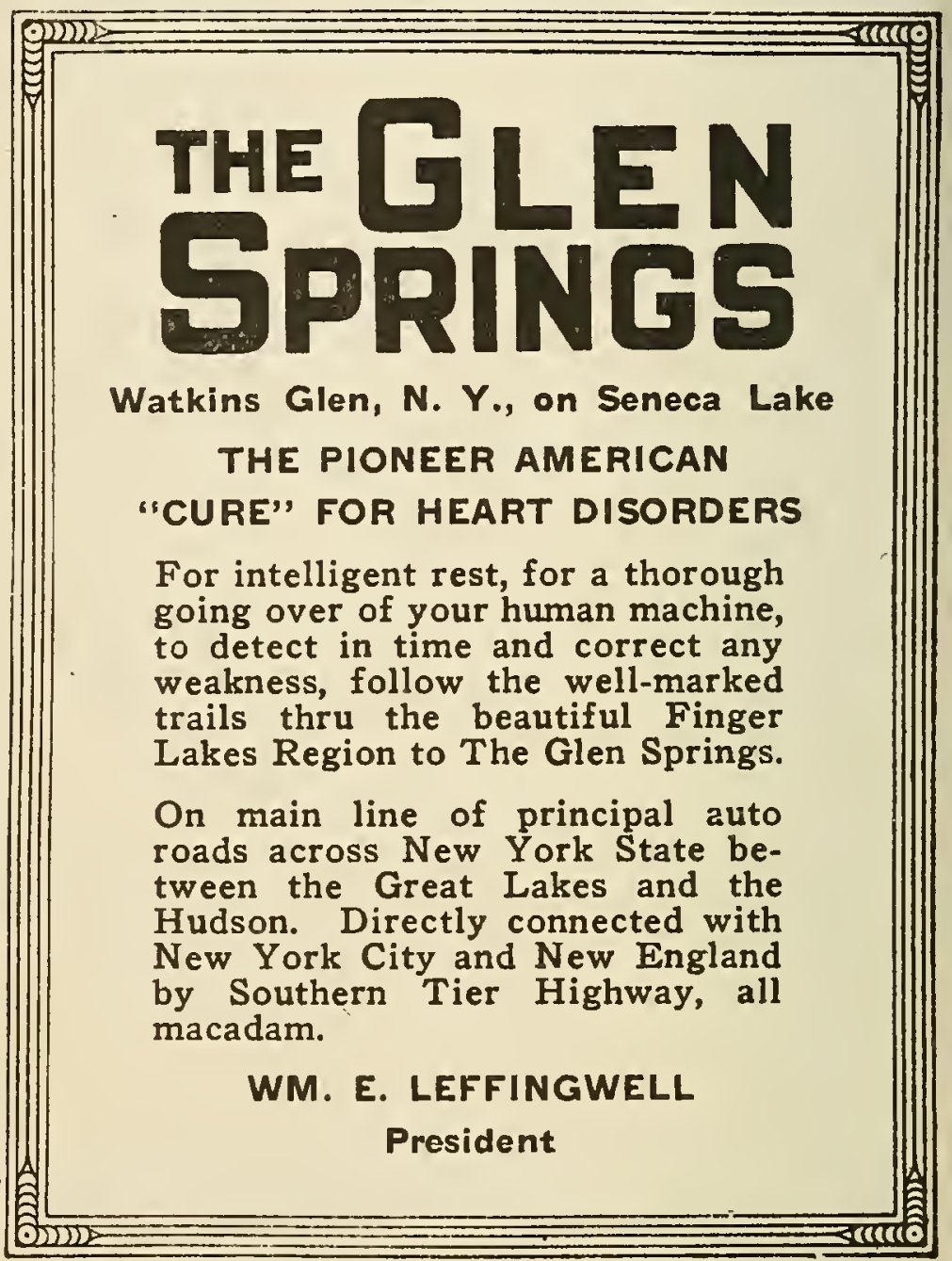

Watkins to Geneva, N. Y., 42.6 Miles

0.0 From Fourth st., Watkins, north on Franl lin.

3.5 Left; right at three corners, Reading Cen

5.7 Left, next right.

8.6 Right at end; left beyond Dundee 13.1 .

13.9 Right fork; right at cross road 17.2 .

24.5 Left; thru Penn Yan 48; to Hall's Corner.

34.3 Half right; right at end 36.1.

36.5 Left at Seneca st.; right at end 38.1.

38.4 Left fork; right on Hamilton st. 40.6.

42.1 Left on Main st. ; right on Seneca st. 42.4.

42.6 Exchange st., Geneva, Hotel Seneca. 
Flmira to Syracuse, N. Y., 90.9 Miles

0.0 From Market St., Elmira, north on Lake

1.2 Left on Division St.; right on Grand Cent Ave. 1.3 .

5.6 Right on Franklin St.; Horseheads; rig fork 6.7 ; keep left 8.2 .

16.2 Right fork after bridge; right 22.0 ; left 29

33.6 Bear left on Cayuga St.; across State Ithaca 34.0.

34.2 Right on Mill St.; left on University A 35.0.

37.8 Left at end; left at Dryden P.O. 46.0.

56.2 Left on Main St., Cortland; half left; rig at end; on Homer St.; thru Homer 59.4.

68.8 Half right at 4-cors.; right at Tully 70.9.

73.2 Left; left at end, Onativia 78.4; right 78.9

74.3 Left down hill thru Onondaga Castle Salina St.

90.0 Syracuse, Genesee St. at Square.

\section{Cortland Thouse}

N. A. Smith \& Co. GORTLAND, N.

Rooms with Hot and Cold Water, $\$ 1.50$

Rooms with Bath, $\$ 2.00$ and up

Meals, Table d'H

BISSELL GAR A GE On the Ley
South STATE AND Syracuse N.Y.

SOUTH STATE AND SUTACUSE, N. Y . Storage Capac
EAS'T WATER STS. SUT Cars

Syracuse to Elmira, N. Y., 90.9 Miles

0.0 From Square, Syracuse, south to Salina St

6.6 Half left; right at end; left at Lafayette

11.6 Right at Onativia; left at Tully 19.5; 1e 21.6; thru Homer 31.5; left with trolley 33. coming into Main St.

34.7 Right on Tompkins St., Cortland; left 43.9

44.9 Right at Dryden; thru Varna 52.6; right 53

55.9 Left on University Ave.; $\mathbf{R}$ on Mill St. 56

56.7 Left on Cayuga St., Ithaca; cross State 56.9.

75.3 Right fork post school; left 81.0 ; to Hors heads.

85.2 Left on Grand Central Ave.; left on Divisi St. 89.6.

89.7 Right on Lake St. to Market St., Elmira 90

\section{LIABLE TO ARREST.}

For violations of the Motor Vehicle Law as follows:

By parking at prohibited places: street intersection; with ten feet of cross walk; ten feet of" hydrant; in front of within fifteen feet of entrance to public building.

By emitting unnecessary smoke.

Approaching, within less than seven feet, a street car rece ing or discharging passengers.

By failing to have vehicle properly licensed. 


\section{Utica to Binghamton, N. Y., 97.2 Miles}

0.0 From Bleecker st., Utica, southwest on Genesee st.

3.5 Right fork, New Hartford; left fork 6.4.

8.9 Right on College st., Clinton; left on Franklin ave. 9.

10.7 L after R.R.; L at Deansboro across R.R. 13.7.

19.1 Right on Main st., Waterville; left fork Sanger st., thru Sangerfield 20.5; right fork 22.1.

28.9 Left at end, Hubbardsville; left fork 32.4.

39.4 Left at monument Sherburne; right over bridge 44.2 .

45.0 Half left at Galena; right fork on Broad st., Norwich 50.3 ; cross Main st., 51.2 ; thru Oxford 59.1 .

63.2 L over bridge; L after bridge at Greene 74.7.

81.8 Left at Chenango Forks; right at Port Crane 90.1.

90.2 Next left into Chenango st. to Court House, Binghamton 97.2.

\section{Binghamton Motor Car Company, Inc.}

(Official Garage for Hotel Bennett)

195-199 WATER ST. BINGHAMTON, N. Y.

\section{USE PLENTY OF OIL AND GREASE}

The pleasure to be derived from automobiling, the safety of the occupants of the car, the saving of hundreds of dollars to every owner, the keeping of appoiitments, all depend upon proper use of oil and grease.

Lubricating System:

Proper Lubrication is more important than any other one item. Many times troubles of a motor car can be traced to lack of lubrication or the use of an inferior lubricant.

No Definite Rule can be followed as to the use of lubricants. These depend entirely upon conditions; naturally, the more the car is used, the more it should be lubricated.

Schedule of Lubrication:

Every roo Miles-Give grease cups a turn or two on springs, rear axle bearings, fans, foot-brake connections, steering knuckles, steering connection, clutch, any about engine. Inspect crank case oil gauge for proper level of oil.

Every 500 Miles-Speedometer shaft, universal joint, selfstarter bearings, spark throttle control, generator bearings, and drive steering column.

Every 1,000 Miles-Screw down all grease cups and refill on springs (also between the leaves), wheel bearings, transmission. magneto, steering gear case, clutch, differential, rear axle.

Every 2,000 Miles-Drain crank case when engine is hot, allowing engine to run a moment to thoroughly cleanse itself

Do not flush out with kerosene as it's impossible in most engines to get the kerosene all out.

Refill with fresh lubricant to proper level.

Drain transmission and thoroughly cleanse with kerosene. Refill with fresh lubricant to proper level.

Drain rear axle and differential and thoroughly cleanse with kerosene. Refill with fresh lubricant to proper level. 
Albany to Binghamton, N. Y., 151.7 Miles

0.0 From State st., Albany, south on Eagle st.

0.4 Right on Madison; left at Dana Memorial on Delaware ave., 0.9 .

2.5 Right at cemetery; left at end thru E. Berne.

25.0 Left at Berne; right fork, Gallupville 31.6.

35.5 Sharp right; left at end 38.1 ; thru Central Bridge 39.

43.7 Right; right fork at park Cobleskill 47.0.

58.6 Right at end; thru Worcester 64.3.

65.4 Left; left under R.R. past Colliers P. O. 79.8.

85.2 Right on Chestnut st., Oneonta.

87.2 Left with trolley; thru Otego 93; Unadilla 103 and Sidney 108.

109.1 Left over river; right at Bainbridge 113.6.

114.0 Left; thru Nineveh 124.

126.5 Left at Harpursville; right at edge of Windsor 136.4 .

146.7 Right into Court st. to Court House, Binghamton 151.7 .

\section{HOTEL ONEONTA}

One of the best, modern, fireproof hotels in the State. Catering

to the motorists-splendid rooms-best of food-prices reasonable. Fireproof Garage attached.

HOTEL PONTIAC OSWEGO, N. Y.

\section{HOTEL KINGSBORO}

GLOVERSVILLE, N. Y.

Under Same Management

H. M. STANFORD, Prop.

Oneonta, N. Y.

F. W. R. HINMAN, Resident Manager

\section{Eat at---KILKENNY'S}

Binghamton to Albany, N. Y., 151.7 Miles

0.0 From Court House, Binghamton, east on Court st.

4.9 Left at end; left, edge of Windsor 15.3.

25.2 Right at Harpursville; thru Nineveh 27.7.

37.8 Right across R.R.; thru Bainbridge; left 38.2 .

48.7 Straight thru Unadilla, with trolley into Chestnut st.

66.5 Left on Main st., Oneonta; thru Colliers 71.9.

86.3 Right at end, thru Worcester 87.4.

93.1 Left; left fork 103.2; thru Cobleskill 104.7.

108.0 Left; left at end 116.3.

120.1 Left fork, Gallupville; right at Berne 126.7.

131.2 Right after E. Berne; left at end 138.3 thru Clarksville.

150.8 Right at monument on Madison; $L$ on Eagle st 151.7 State st. at Capitol, Albany. 


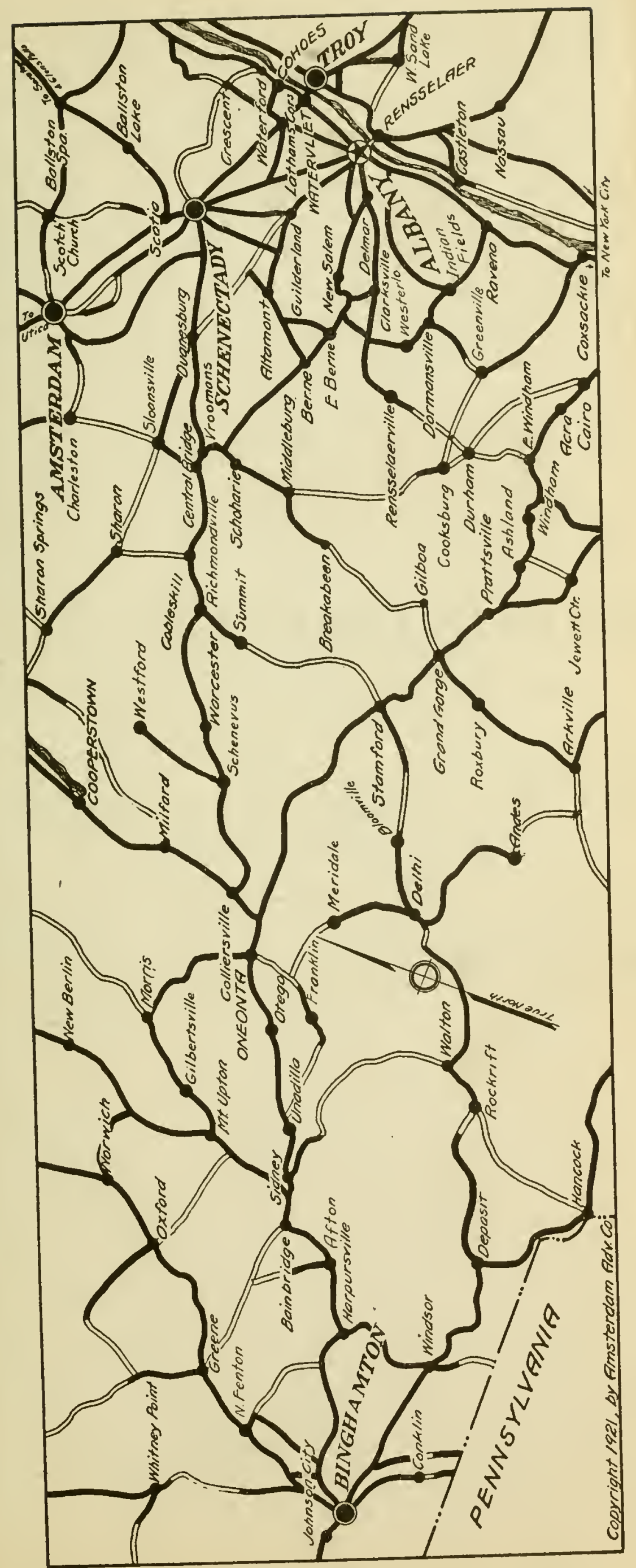




\section{Binghamton to Utica, N. Y., 97.2 Miles}

0.0 From Court Ho., Binghamton north on Chenango st.

7.1 Right at end, next left at Port Crane P. O. 15.3 Right over bridge, again right at Chenango Forks beyond.

22.5 Right over bridge at Greene; follow Norwich signs.

34.0 Right after bridge; thru Oxford 38.2 into Broad st.

45.9 Cross Main st., Norwich; left fork 47.5.

52.2 Half right at Galena; left after bridge 53.

58.7 Right fork beyond Sherburne; right at Hubbardsville 68.3 .

70.1 L fork; thru Sangerfield 76.7; into Sanger st.

78.1 Left at Waterville; right at Deansboro 83.5.

86.5 R across R.R.; thru Franklin Springs 87.5.

88.2 Right at end on College st.; left on Utica st., Clinton 88.3.

92.8 Curve right across R.R. join trolley into Genesee st.

97.2 Genesee st. at Bleecker st., Utica.

\section{BA GGS HOTEL Utica, N.Y. . EUROPEAN PLAN}

First-Class Restaurant in Connection POPULAR PRICES

NHAT THE AUTOMOBILE CLUBS OF NEW YORK STATE HAVE ACCOMPLISHED

I-They have exposed and prevented the operation of uestionable accessory and tire concerns.

2-Were the first to urge the building of good roads in Jew York State, and our 8,000 miles of paved highways are argely the result of co-operation by the New York State Iutomobile Clubs.

3-They have secured reciprocity in Motor Licenses beween this and other states, including Canada.

4-As custodians of the interests of the motorists of the itate, have continuously fought without hesitancy any unair legislation, with the result that not one single law among le hundreds affecting the motorist, introduced each session, ras ever passed in the last ten years when opposed by the Jew York State Automobile Clubs, with one exception. 


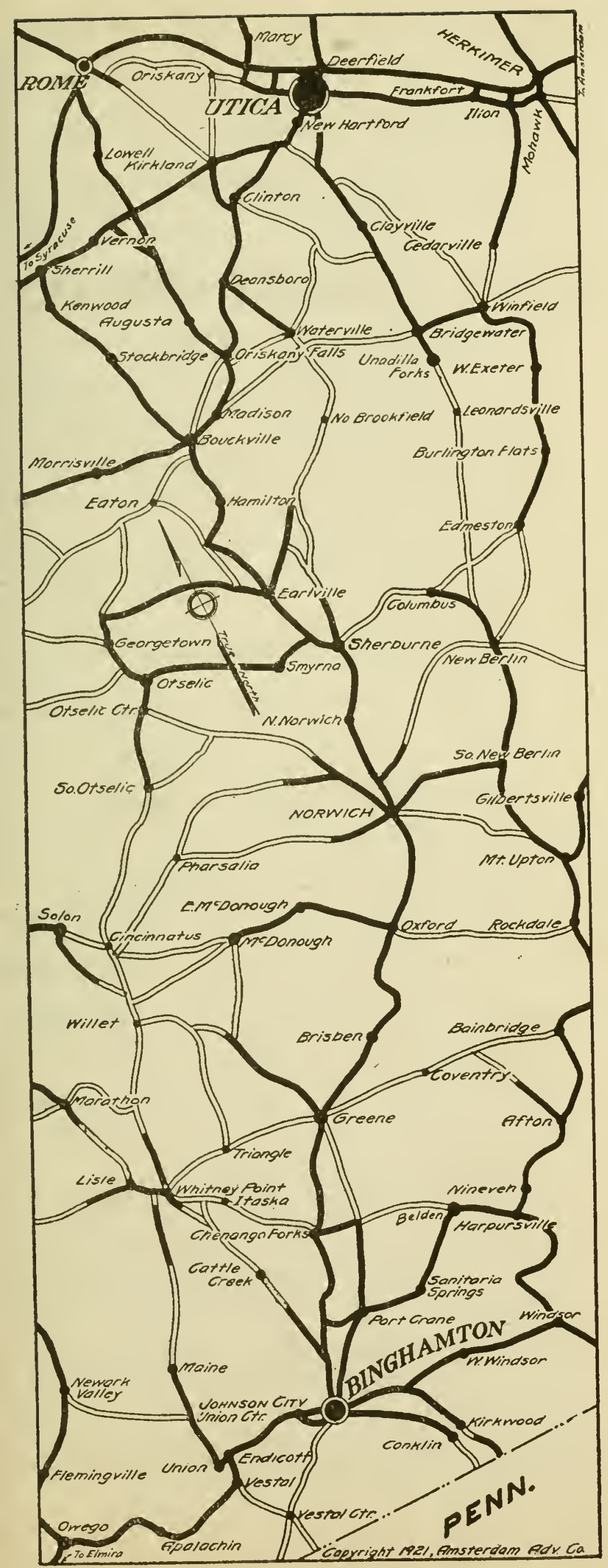


Elmira to Binghamton, N. Y., 59 Miles

0.0 From Main st., Elmira east on Water st.; right after bridge 1.3 ; thru Chemung 13 ; Waverly 19 ; and Barton 24.

31.2 Right at Tioga; right 35.4 ; into Main st., Owego.

36.7 Right on Court st.; across bridge; left at end.

50.0 Left at Vestal; left across river; right at end 50.7 ; to Washington st., Binghamton 59.0.

\section{JUNE AUTOMOBILE COMPANY}

\section{Storage for Hotel Bennett \\ BINGHAMTON, N. Y.}

\section{PEERLESS, DORT and GRANT CARS}

\section{Binghamton to Elmira, 59 Miles}

0.0 From Washington st., Binghamton, west on Main st.

8.3 Left at Union, across river; right at Vestal 9.

21.9 Right across bridge into Owego; right-left 22.2 .

22.3 Left on Main st.; left at end 23.6; right 25.5.

27.8 Left at Tioga P. O., thru Smithboro 33 ; and Barton 35.

36.5 Left at end; into Chemung st.; thru Waverly 41.3.

57.7 Left at end on Water to Main st., Elmira 59.

\section{Elmira to Dansville, 68 Miles}

0.0 From Water st., Elmira, north on Main st.; left fork on College Pl. along R.R.

2.5 Left at end on Central ave., Elmira Hgts.

4.9 Left under R.R.; left over R.R. bridge; right 8.6, thru Big Flats 10, and Corning 18.0.

19.0 Left with trolley thru Painted Post 21; Bath 39.

42.1 Left at Kanona across bridge; next right; right 44.4; across bridge; at once left thru Avoca 47.0 .

54.9 Left at Cohocton; right fork 55.5; right fork 64.0.

68.0 Dansville Main st. at Ossian.

\section{Dansville to Elmira, N. Y., 68 Miles}

0.0 From Ossian st., Dansville S. E. on Main st.

2.2 Right fork; left fork 4.9; left at 3-cors. 12.5 .

13.1 Right at Cohocton; thru Avoca 21; right across bridge at once left 22.6 ; left across bridge.

25.9 Right at Kanona; left on Steuben st., Bath 28.7 ; thru Savona 35 ; Painted Post 47.

49.0 Right with trolley; thru Corning 50; Big Flats 58.

65.5 Right at Elmira Heights coming into Main st.

68.0 Elmira at Water st. 


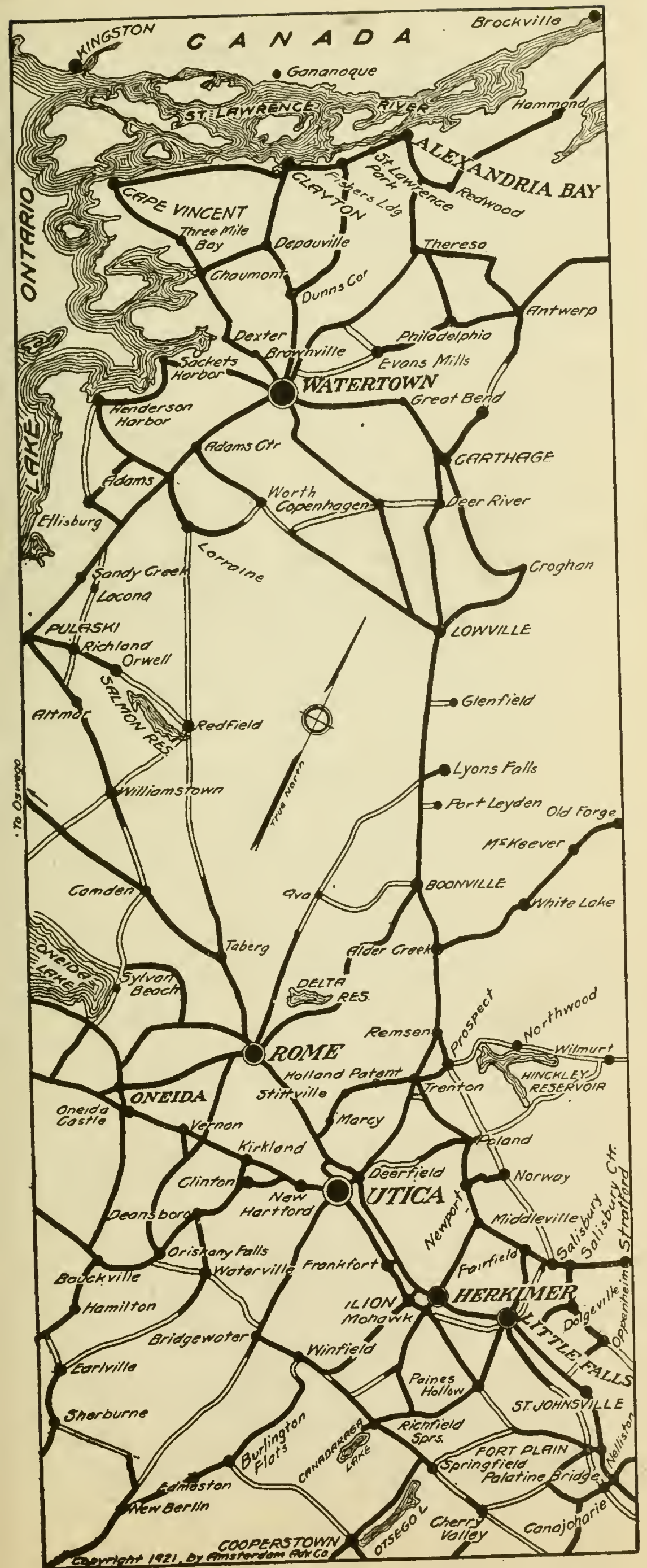


Utica to Watertown and Alexandria Bay, N. Y., 138.3 Miles

0.0 From Bleecker st., Utica, northeast on Genesee.

1.4 Left at Deerfield; right 6.5 ; left 7.3 ; leftright 8.4 .

13.3 Right; left at Barnveld 19.1; thru Remsen 23.

36.1 Left at park, Boonville; right fork Post st.

59.5 Left on Dayan st., Lowville; right at cross road 63.6.

72.0 Left fork at mon., Copenhagen; right 83.

86.0 Right at end, next left along park, Watertown.

86.2 Half right on Court st.; left fork Coffeen st. 86.4.

93.4 Right at cross-road; into Dexter; right 93.8.

95.4 Left over bridge at Limerick; thru Chaumont 100.3 .

112.3 Right on Broadway, Cape Vincent, thru Clayton 127.

138.3 End of Otter st. at James st., Alexandria Bay Note.-For Alexandria Bay via Clayton, keep right on Court st. 86.4. Left on Main st. 86.9 ; right on Bradley st. from trolley 87.1 thru Depauville 100.4. Rignt-left at cemetery 106.4, in James st. Right at State st.., Clayton 107.7, direct to Alexandria Bay, 119.1.

\section{THE CARLETON}

On the St. Lawrence

The Home of the finest Black Bass Fishing in America Modern

ANDREW F. KEOUGH

Cape Vincent, New York

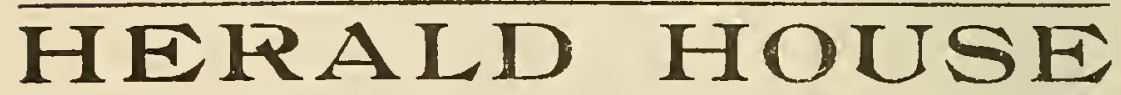
On the St. Lawrence

Glayton, N. Y. American plan-Our table will please you

C. B. BERTRAND \& SON

\section{Devendorf's Garage}

300 car capacity

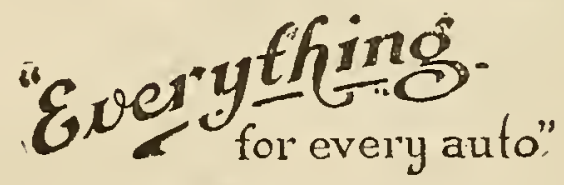

NEVER CLOSED

Next door to Post Office

WA TERTOWN, N. Y. 
Alexandria Bay to Rouses Point, N. Y.

0.0 From Church st., Alexandria Bay, S. E. on James.

6.4 Left at end; left at Redwood 6.9; thru Hammond 19.

29.0 Pass monument Morristown; turn right 29.3. Follow trolley into Main st. across long bridge over Oswegatchie River into Ford st.

40.3 Right on State st., Ogdensburg; half left on Canton st. 40.8 .

58.8 Straight thru Canton; into Maple st., Potsdam.

69.9 Left at end on Water st., next right on Elm.

86.6 Right at P.O. Nicholville; next right.

91.5 Right; thru Moira 98.3 and Malone 111.6.

122.9 Right at 5-corners; thru Chateaugay 124.

126.4 Right under R.R.-left; left 135.8 thru E1lenbutg.

142.6 Left; right at Cannon's Cors., 145.7.

150.6 Left; left after R.R. at Mooers 144.4.

158.8 Right at end; half left over bridge in Champlain; then right fork.

162.5 Left at end, to Custom House, Rouses Point 167.2.

\section{THE MARNES INN}

On Lake Ghamplain, Rouses Point, New York Near Post Office-Cobble Stone Garage, American plan, excellent appointments

Boating - Bathing - Fishing M. A. PHILLIPS, Manager

Rouses Point to Alexandria Bay, N. Y.,

0.0 From Custom Ho., Rouses Point, South along lake.

0.7 Right; right fork in Champlain 5.1; left 8.6.

13.2 Left, curve right into Mooers.

14.0 Right at end; right at end, Mooers Forks 17.

17.4 Half left across bridge; left across river 22.

25.0 Right at end thru Ellenburg 28; right 30.8 .

42.1 Right under R.R.; left thru Chateaugay 43.6.

44.1 Left fork of 3 roads; left at 5-corners 44.7 ; thru Burke 48.5 into Main st., to monument at hotel, Malone 56. Thru Moira 69.3.

76.1 Left at 4-cors.; left at Nicholville 81.

97.6 Left at end, Potsdam; next $\mathbf{R}$ on Maple st.

99.5 Left fork at sign, thru Canton 108.6.

108.8 Keep right direct into Canton st., bear right into State st.

127.2 Left on Ford st., Ogdensburg, bear left with trolley across Oswegatchie River bridge; bearing right beyond.

127.7 Left after R.R., next right on Main st.

138.3 Left at end, past monument, Morristown.

160.7 Right at Redwood; again right 161.2 into James st.

167.6 Church st., Alexandria Bay. 
Alexandria Bay to Syracuse, N. Y., 100.4 M.

0.0 From Church st., Alexandria Bay, southeast on James st.

4.0 Right; thru Plessis 6; left at school 10.3.

11.6 Right on Main st., Theresa; right 12.6 ; thru Pamelia 21.4.

30.4 Half right on Washington St, at park, Watertown.

32.7 Right fork; left fork 34.2; thru Adams Center 40.7.

44.6 Right on Grove st., Adams; left 47.1.

53.8 Right fork; left fork 54.3 to park, Pulaski.

63.0 Sharp left over bridge, at once right; thru Mapleview 71; Hastings 76.4; Brewerton 86.4; Cicero 90.4.

98.8 Left on Salina st.; half right 99.1.

100.4 Syracuse, Clinton Square, at monument.

THE YATES SYRAGUSE, N. Y. Modern in Every Respect

EUROPEAN PLAN

Rooms with or without Bath and En Suite Unexcelled Dining Room

Club Breakfasts

Courtesy and Fairness

- Member of Empire Tours

WILLIAM D. HORSTMANN

BISSELL GARAGE On the Level SOUTH STATE AND SYracuse, N.Y. Storage Capacity EAST WATER STS. NYLACUSE, N. Y. 400 Cars

The MU12\% E. L. BRIDGES, Manager

South Warren and Harrison Streets

SYRACUSE, N. Y.

Designed and operated to make it a Hotel of Refinement for Discriminating Guests

EUROPEAN PLAN

RATES: Rooms without Bath $\$ 2.00$ per day

Rooms with Bath $\$ 2.50$ per day and upwards

\section{Liable to Arrest}

For violations of the Motor Vehicle Law as follows:

Driving at a rate of speed constituting "Reckless Driving."

Failing to give proper signal upon approaching intersecting street.

Not giving sufficient signal upon changing course of rehicle.

By failing to grant proper right of way.

Driving or parking on wrong side of street.

By having muffler cut-out open. 
Syracuse to Watertown, N. Y., 70. Miles

0.0 From Square, Syracuse, north on Salina; L 1.3.

1.6 Right on Wolf St. thru Cicero 10.; Brewerton 14.; Hastings 24.; Mapleview 29.5.

37.3 Left at end across bridge; next sharp right at park thru Pulaski 37.5.

42.4 Sandy Creek straight thru Pierrepont Manor 50.

50.3 Right at bridge; right at 5-corners 53.3.

55.8 Left at 4-corners on Main St., Adams.

69.7 Right fork at P.P., Adams Center; left 69.9.

70.0 End of Washington St. at park, Watertown.

Watertown to Syracuse, N. Y., 70. Miles

0.0 From park Watertown, south on Washington St.

2.3 Right fork; L fork 3.8; thru Adams Cen. 10.3.

14.2 Right at 4-cors. on Grove St., Adams; L 16.7.

23.4 Right fork; left fork 23.9 to park, Pulaski.

26.4 Sandy Creek straight thru.

32.6 Sharp left across bridge; at once right; thru Mapleview 40.5; Hastings 46.; Brewerton 56.; Cicero 60.; bear right into Wolf St.

68.4 Left on Salina St. to Square, Syracuse 70.

\section{E ISSELL GARAGE On the Level S' JTH STATE AND.
EAST WATER STS. Syracuse, N.Y.}

Syracuse to Oswego, N. Y., 35.7 Miles

0 . From square, Syracuse, north on Salina; left 1.3.

1.6 Right on Wolf st.; next left on Park st.

5.0 Half right from trolley, Liverpool, on Oswego st.

12.9 Right, cross Oneida River, thru Phoenix 15, and Fulton 24.2.

35.7 Bridge st., Oswego.

\section{HOTEL PONTIAC}

One of the best, modern, fireproof hotels in the State. Catering

to the motorists Splendid rooms. Best of food.

\section{Prices Reasonable---Good Garage}

Hotel Oneonta, Oneonta, N. Y. Hotel Kingsboro, Gloversville, N. Y., under same management. 
Alexandria Bay to Utica, N. Y., via Cap Vincent and Watertown, N. Y., 138.3 M.

0.0 From James st., Alexandria Bay, southeast o Otter st., thru Clayton 11.3 into Broadwa Cape Vincent.

26.0 Left on Market st. thru Chaumont 38, int Limerick.

42.9 Right after bridge; half left 43.1 ; left 44.5 Dexter.

44.9 Left at cross road; bear right into Court s at trolley.

52.1 Half right on Washington st., Watertown next left along park,

52.3 Left-right into State st.; left at end 55.6 thr Copenhagen 66.3 .

75.7 Left; right on State st., Lowville 78.8; thru Turin 91.3.

92.2 Right fork Fisher st., Boonville; L after R.R. 119.3 $\mathrm{R}$ at end, Barnveld; $\mathrm{L} 125$; left-right 130 131.1 Right at cross road; left 131.8; right at Deerfield 136.9; on Genesee st. to Bleecker st., Utica 138.3.

\section{HOTEL WESTMINSTER}

\section{Alexandria Bay, N. Y. \\ YE WALTON INN}

Glayton, N. Y.

In the "Heart of the 1000 Islands." Fine boating, bathing, fishing, golf, tennis, polo, music, dancing and many other indoor and outdoor attractions. Write for latest automobile road map of the Great North Country.

Long Lake to Chestertown, N. Y., 55.3 MI.

0.0 From Long Lake, run east, thru Newcomb 14, and Minerva 33.

40.3 Left at end, North Creek; left at Wevertown 45.7.

49.3 Left acrus: R.R.; right fork 51.2.

55.3 Main st., Chestertown.

\section{LAKE HARRIS HOUSE}

ON LAKE HARRIS, IN CENTER OF ADIRONDACKS

A modern summer hotel. 6,000 acres of lakes and forests; free hunting and fishing privileges to guests; gas, steam heat and open fireplaces; telegraph and Bell telephone; mails daily; autobus from North Creek.

\section{Now Open New Management}

JOSEPH H. GREGORY, Prop., Newcomb, Essex Co., N.Y. 
Rochester to Niagara Falls, N. Y., 93.3 M.

0.0 From Main st., Rochester, north on State st.

2.9 Left on Ridge rd.; thru Greece 6; Parma 11.

18.4 $\mathrm{L}$ at Clarkson; $\mathrm{R}$ at edge of Brockport 19.5.

24.2 Right on Mechanic; left on Wright; bear left on Albion st.; Holly 24.4; right 27.3; thru Albion 34.

44.1 Right on Center st., Medina; right on Prospect 44.6 .

47.9 Left at Ridgeway; thru Hartland 58.

Wright's Cor. 64.3 into Market st., Lockport.

67.9 Right on Main st. with trolley thru Sanborn 81.2.

89.7 Left at end; jog right-left into Tenth st., 91.5.

92.5 Right on Buffalo ave. to Main st., Niagara Falls 93.3.

THE HOTEL IN LOGKPORT Is the

HOTEL KENMORE

Rates $\$ 4.00$ Upwards

AMERICAN PLAN

\section{CLARK GARAGE}

STORAGE and SUPPLIES

We sell and distribute the well-known Kendell Gasoline and Kerosene

ROY K. CLARK, Lockport, New York

After viewing the Falls visit a most instructive and interesting feature

NIARAGA FALLS MUSEUM

Price Twenty-five Cents

\section{TEMPERANGE HOUSE}

WM. T. PHELPS, Proprietor

NIAGARA FALLS, N. Y.

European and American

Plan

Hot and Cold Water in Every Room

75 Rooms with Bath

TABLE SUPPLIED FROM OUR OWN FARM

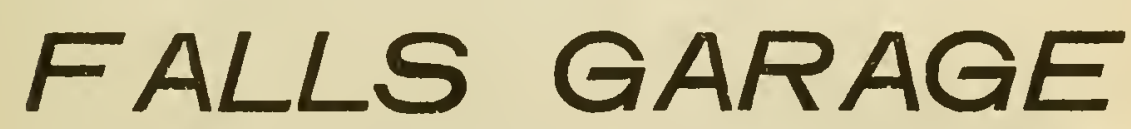

New fire-proof garage. Centrally located

NIAGARA FALLS, N. Y.

Capacity 300 
Niagara Falls to Rochester, N. Y., 93.3 M.

0.0 From Main st., Niagara Falls, east on Buffalo ave.

0.8 Left under R.R. on 10th st.; jog right-left 1.5.

3.8 Right after R.R. Whirlpool ave; thru Sanborn 12 .

12.3 Left on Market st., Lockport with trolley; left over canal 26.4 .

29.0 Wright's Corners; keep right; $\mathbf{R} 32$; thru Hartland 35.3.

45.4 Right at Ridgeway; half right on Prospect 48.5.

48.8 Left on Center st., Medina; L on State st.49.2. 37.9 Right on East ave.; thru outskirts Albion 59.3. 68.9 Half right at Holly; left on State st. 69.2.

70.6 Left over canal; left 73.8; $\mathrm{R}$ at Clarkson 78.9. 90.4 Right at end on Lake ave. into State st. to Main st.. Rochester 93.3.

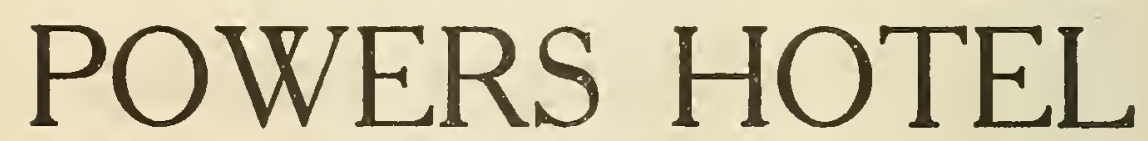

ROCHESTER, N. Y.

The Hotel of

"COMFORT, CONVENIENCE and COURTESY"

Renowned Dining Facilities, including Cafeteria Service

Make "THE POWERS" your "HEADQUARTERS" during the noon stop-over, or after the day's run

\section{OVERLAND SALES CO. Lockport, N. Y. \\ Phone 1460 \\ Opposite Big Bridge}

Storage - Supplies - Parts OVERLAND WILLYS KNIGHT AUTO REPAIRING

SENEGA GARAGE ALWAYS OPEN

Around corner from the Seneca, Whitcomb, Hayward and Osburn Hotels

ROGHESTER, N. Y.

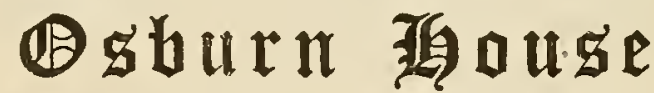

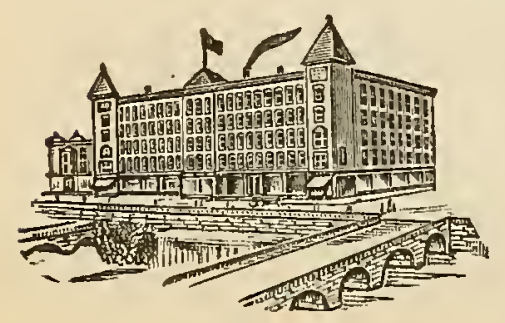

ROGHESTER, N. Y.

American plan, $\$ 3.50$ up

Headquarters U. C. T.

Free Garage 
Oswego to Syracuse, N. Y., 35.7 Miles

0.0 From Bridge st., Oswego south on 4th; thru Fulton 11.5 and Phoenix 20.7.

22.8 Left after bridge, Oneida River.

30.7 Half left at trolley, Liverpool.

34.0 Right on Wolf st.; next left on Salina.

35.7 Square, Syracuse.

\section{BISSELL GARAGE On the Level SOUTH STATE AND
EAST WATER STS. Syracuse, N.Y.}

\section{Rochester to Hornell, N. Y., 68.4 Miles}

0.0 From Main st. Rochester south on Plymouth.

0.9 Left on Clarissa st.; right after bridge on Mt. Hope ave.

18.1 Right at East Avon; left at square, Avon 20.1

20.5 Right on Spring st.; left on Wadsworth 21. 29.1 Half left on Main st., Geneseo.

29.5 Right at end; right at end 33.5.

35.6 Left on Main st., Mt. Morris.

50.3 Left on Ossian st.; right on Main st., Dansville 50.8 .

50.9 Right on Exchange st.; thru Arkport 63.

63.5 Left; left at end 65.4.

65.6 Right over bridge into Seneca St.

68.4 Main st., Hornell.

\section{HOTEL SHERWOOD}

American Plan-Modern

Quiet-Reasonable

E. M. CHASE, Hornell, N. Y.

Hornell to Rochester, N. Y., 68.4 Miles

0.0 From Main st., Hornell, north on Seneca.

2.8 Left after bridge; right 3.0 ; right at end 4.7 ; thru Arkport 5.4.

17.3 Left on Main st., Dansville.

17.4 Left on Ossian st.; right on Dack st., 17.9.

32.8 Right on State st., Mt. Morris.

34.9 Left with travel; right, next left 38.8 .

39.3 Half right at C. H., Geneseo.

47.4 Right on Spring st.; left on Genesee 47.9

48.3 Right at square, Avon into East Avon.

50.3 Left at tavern; thru West Henrietta 60.

67.3 Left over bridge; right on Plymouth 67.5

68.4 Main st., Rochester.

Tell them that you saw it in Motor Tours 
Watertown to Rochester, N. Y., 131.0 Miles

0.0 From park, Watertown, south on Washington st.

2.3 Right fork; left fork 3.8; Adams Center 10.3.

14.2 Right on Grove st., Adams; left 16.7.

23.4 Right fork; left fork 23.9 to park, Pulaski.

32.6 Sharp left across bridge; at once right into Mapleview.

40.6 Right at four corners across railroad; thru Mexico 45.

45.8 Right fork; thru Scriba 45.7; into Oneida st.

59.3 Right on Tenth st., next left on Bridge st.

60.2 Cross First st., Oswego; half left on Seneca 60.9.

68.9 Left at smithy; left after bridge; right 72.1, thru Sterling Center 72.7; Fairhaven 76.2.

81.5 Right at three corners, Red Creek; left at end 83.

87.6 Right at end, Walcott; thru Alton 96.7; Sodus 101.

123.4 Left at West Webster; left 128.3; right 129.1; left 129.8 on Webster ave.

130.0 Bear left on Goodman; half right on Main st. to Clinton st., Rochester, 131.

MEXICO a village of about 2,000 on the Roosevelt Hotchkiss Hotel and Restaurant is where Tourists are treated right. It's like home - mother does the cooking and prices are moderate. Wash your hands, comb your hair and sit right down. No tiresome waiting. Main Street, next to Garage.

F. E. HOTCHKISS, Prop.

\section{Mexico Motor Car and Supply Co.}

Competent Mechanics MEXICO Storage and Supplies ROCHESTER TAXICAB COMPANY

F. J. ZORN, President and General Manager

Close to Powers Hotel and Hotel Rochester

Five minutes from Main St. - 58-64 Plymouth Ave., North

Rochester to Watertown, N. Y., 131.0 Miles

0.0 From Clinton st., Rochester, northeast on Main st.

1.2 Half left on Goodman; half right on Webster.

2.4 Left at end; right on Clifford st. 3 ; bridge 4.7.

7.6 Right at trolley, West Webster; thru Sodus 30.3 ; Alton 34.

43.4 Left across bridge at Walcott; left 47.

49.6 Left at three corners, Red Creek; thru Fairhaven 55.

58.9 Left at trough; right at end; right at smithy 62.1.

70.1 Half right on Bridge st. thru Oswego 71.

71.7 Right from trolley on Tenth; next left on Oneida thru Mexico 86; turning half right 86.1.

90.4 Left after railroad, Mapleview; left at end across bridge; next sharp right at park, Pulaski 98.2.

111.2 Right at bridge; right at five corners 114.3.

116.7 Left at four corners on Main st., Adams.

120.6 Right fork at postoffice, Adams Center; left 128.7.

131.0 End of Washington st. at park, Watertown.

\section{LUBRICANT FOR SPRINGS}

In lubricating automobile springs the greatest trouble is found in keeping the lubricant from being squeezed out under pressure. A compound that obviates this is found in graphite and beeswax mixed to a paste and spread upon each leaf. Obviously, the spring must be taken apart to apply this lubricant. 
0.0 From Main st., Niagara Falls, S. E. on Buffalo ave.

0.9 Left under R.R. on 10th; right on Pine st. ave. 1.5

4.8 Left across Canal; right at trolley on Main st. at monument, Buffalo 26.6 miles.

\section{ull Course Luncheons $50 \mathrm{c}$. \\ 11 A. M. -2.30 P. M.

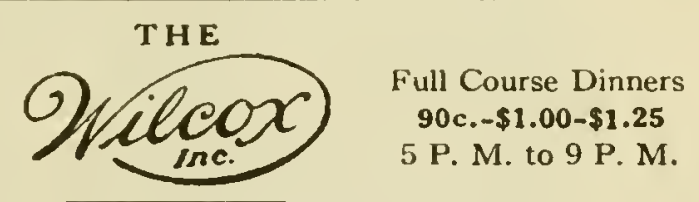

Restaurant - A Ia Carte Service at all hours

Music to $2 \mathrm{P} . \mathrm{M}$. 287-289 WASIIINGTON ST. (near Swan) BUFFALO, N. Y.

Music $6-8$ P. M.

uffalo to Niagara Falls, N. Y., 26.6 Miles 0.0 From mon., Buffalo, north on Main; left on Bvd. 5.5.

5.7 Right at race course; left at end 11.6.

25.1 Left on 10th st.; right at end after underpass ; on Buffalo ave. to Main st., Niagara Falls 26.6.

he real attraction at Niagara Falls is the MUSEUM and BAZAAR The Oldest Dealers

\section{TOURIST! A Home for Your Car UPPER'S GARAGE, Inc.}

40 Second Street, Niagara Falls, N. Y.

One half block from leading Hotels $\left\{\begin{array}{l}\text { Prospect House } \\ \text { Imperial } \\ \text { Temperance Ho }\end{array}\right.$

Temperance House

Dansville to Buffalo, N. Y., 77.3 Miles

0.0 From Main st., Dansville west on Ossian; right 0.5 .

15.2 Mt. Morris, cross State st.; L 16.5; L 18.7.

24.8 $\mathrm{R}$ on Covington st., Perry; L at Perry Cen.

33.6 Right on Court; left on Main; right on Buffalo st., Warsaw; thru East Aurora 60.

60.5 Right fork; thru Ebenezer into Seneca st.

77.1 Right with trolley on Main st. to mon., Buffalo 77.3 .

\section{Buffalo to Dansville, N. Y., 77.3 Miles}

0.0 From Main st., Buffalo, east on Swan st. thru Ebenezer 8; and East Aurora 17.3.

43.3 Left on Main st.; right on Court st.; left on Park st., Warsaw.

50.6 Right at Perry Cen.; left on Liberty st., Perry.

58.6 Right; right at end 60.8; thru Mt. Morris 62.1 .

77.3 Dansville, Ossian st. at Main. 
New York to Greenport, L. I., 107.1 Miles

0.0 From Automobile Club of New York, Hote. Ansonia, South on Broadway.

0.1 Left on 72nd St.; thru Central Park into Fifth Avenue.

1.5 Left on 57 th St.; left on 2 nd Ave.; right on 59th St.; across Queensborough Bridge.

3.6 Left beyond Plaza on Jackson Ave.; thru Flushing 9.3 ; under R.R. at Broadway Sta. 10.8 ; thru Bayside 12.8 .

17.1 Right fork Manhasset; $\mathrm{L}$ at clock tower 20.3.

20.5 Left at end Roslyn; right fork; right 20.8 .

31.4 Left at 3-cors.; right fork 33.; half left 33.9 ; thru Huntington 34.3 ; and Centerport 37.3.

39.6 Right at end; left at cross rd. 43.2 ; thru Commack 44.7.

49.6 Left at cross rd.; L at end; thru St. James 53.3.

57.1 Right, thru Setauket; $\mathrm{R}$ at Port Jefferson 60.3 .

59.1 Left at cross rd.; thru Shoreham 70.8.

73.8 Right fork, Wading River; left at end 77.6.

87.1 Left at end, Main St., Riverhead; thru Jamesport 89.3 ; Cutchogue 56.; and Southold 102.; into Front St. to

107.1 Main St., Greenport, L. I.

Greenport, L. I., to New York, 107.1 Miles

0.0 From Main St., Greenport, west on Front, thru Southold 5.; Cutchogue 10.; and Jamesport 17.

22.3 Right fork, Riverhead; right 25.; right 25.8 .

33.3 Left fork, Wading River; thru Shoreham 36.3 .

45.9 Right on Main St.; left on Broadway, Pt. Jefferson 47.

49.9 Left thru St. James 54.; right 55.3; thru Commack 62 .

63.6 Right at cross rd.; left 67.6; thru Centerport 70.; Huntington 75.; and Cold Spring Harbor 75 .

75.7 Right at small bridge; right at Roslyn hotel 86.5 ; thru Manhasset 90. ; Bayside 94.3 ; and Flushing 97.8.

103.5 Right across Plaza; over Queensboro bridge.

105.2 Right on Second Ave.; next left on 60th St.

105.7 Half right into Central Park, left fork at Mall, left at mon., next right out of Park into 72 nd St.; right on Broadway.

107.1 Automobile Club of New York, Hotel Ansonia on left.

It takes brains to build a good article or find a better way to do things! Any one can cut prices. Tires and tubes-happiness in every ride. Will save you money on guaranteed tires and tubes. Only costs a cent to get prices.

AMSTERDAM SUPPLY CO., Amsterdam, N. Y. 


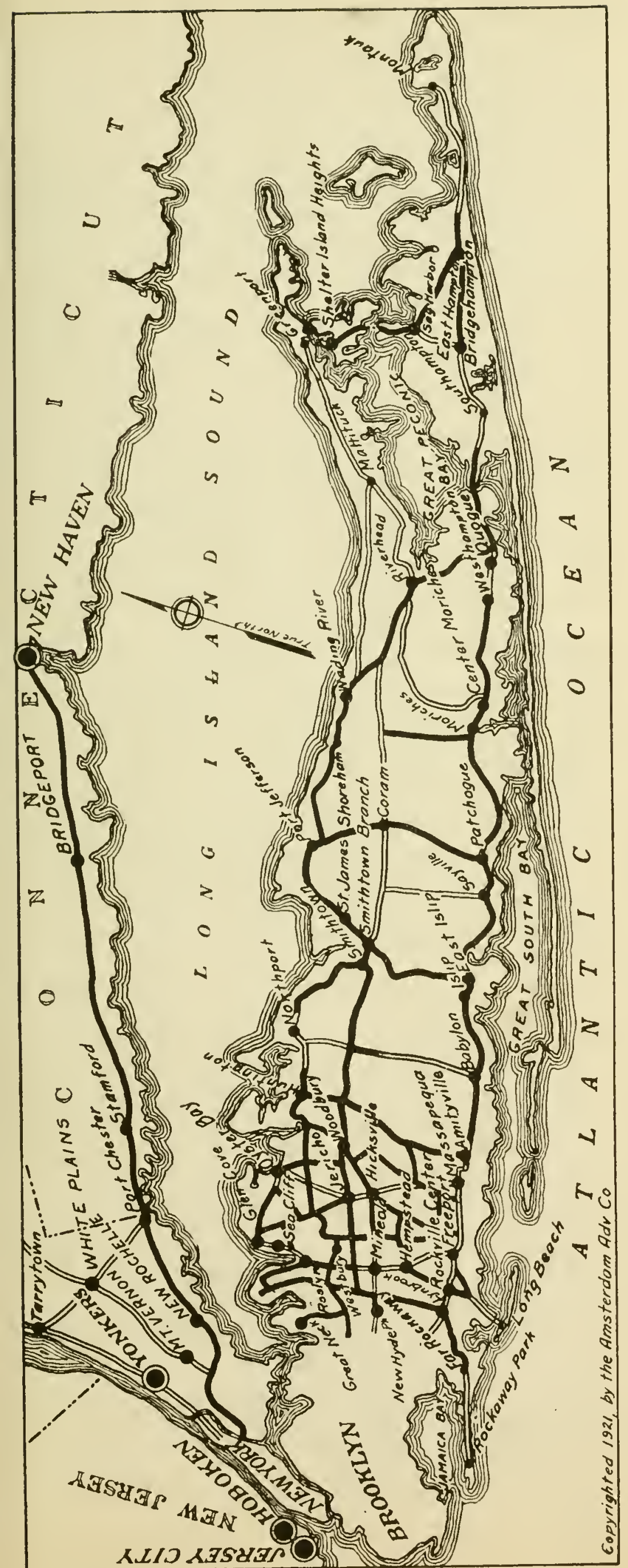


New York to Amagansett, L. I., 113.0 Miles

0.0 From Automobile Club of New York, Hotel Ansonia, south on Broadway.

0.1 Left on 72nd St.; thru Central Park into Fifth Avenue.

1.5 Left on 57th St.; left on 2nd Ave.; right across Queensboro Bridge; keep right across Jackson Ave.; over R.R. viaduct. Keep right fork, Winfield, thru Forest Hills.

11.2 Left on Hillside Ave.; right at Peace mon., Jamaica 12.3.

19.9 Half left at Lynbrook; thru Rockville 21.; Freeport 25.; Babylon 39.; Bayshore 44.; and Islip 45.

54.2 Left fork, Sayville; thru Patchogue 58.; and Moriches 70.

76.8 Right, thru Eastport; half L 78.4; L fk 81.4.

83.9 Right into Quogue; left at hotel 84.6; thru Good Ground 91.4; into Southampton.

98.6 Left on Main St.; next R; right fork 100.6. 103.8 Right fork beyond flagpole, Bridghampton.

111.6 Right fk, E. Hampton to Amagansett 113.0.

Amagansett to New York City, 113.0 Miles

0.0 From Amagansett, $\mathrm{E}$ thru Easthampton 1.4.

9.2 Keep L at Bridghampton; L on Main St. 14.3.

14.4 Right at Southampton; right fork 14.6; thru Good Ground 21.6.

23.1 Right after R.R.; thru Quogue 28.4; left 29.3.

36.3 Left at end; thru Moriches 42.6 right fork 48.3; thru Patchogue 54.6; Sayville 59.; Islip 67.; Bayshore 69. ; Babylon 73.6; Freeport 88.; and Rockville 91.6.

93.1 Half right at Lynbrook, across Fulton St., Jamaica.

101.8 Left at Peace mon.; right on Queens Bvd. Manhattan.

110.8 Right at end; next left on 60 th St. to Fifth R.R. viaduct; across Queensboro Bridge into 112.8; thru Forest Hills 104.; half R over Ave.

111.6 Half right into Central Park; left fork at Mall; left at Webster mon., next right into 72nd St.; right on Broadway.

113.0 Auto Club of New York, Hotel Ansonia.

\section{HOTEL EMPIRE} BROADWAY AT 63rd STREET

Centrally located near all the Famous Shops and Theatres

The Hotel of Easy Access

Our Restaurant is noted for its excellent food and moderate prices

P. V. LAND, Manager 
New Xork to Philadelphia, 99.4 Miles

0.0 W. 42nd st., ferry to Weehawken.

0.6 Bear right at top; left on 5th st. 0.7.

1.2 Left-right; left at end on Boulevard 1.4.

6.7 Right into park; left at circle; keep left.

7.4 Right at trolley; right on Market 10.9 .

12.1 Cross Broad st., Newark; left fork at C. H.

12.6 Left on High; right at trol. on Clinton.

13.6 Left on Elizabeth ave. with trolley.

17.9 Right at C. H. Elizabeth on Rahway ave.

25.0 Right from underpass (under const. 1919). During const. straight on, right at Woodbridge.

31.2 Left over R.R.; at once right, Metuchen.

35.9 Left at P. O., New Brunswick, on George.

36.5 Right on Commercial; left with trol. 37.

54.0 Right fork, Hightstown, to Robbinsville.

60.2 Right across R.R., right fork 64.2.

66.0 Left on State st., Trenton, across Broad.

68.6 Left on Calhoun across Delaware R. (20c.)

71.5 Left under R.R., then right.

86.7 Half right, Bustleton; right fork 88.

94.8 Left on Broad st., Philadelphia.

99.4 Around City Hall; into S. Broad st.

Philadelphia to Baltimore, 103.7 Miles

0.0 From Broad st., Philadelphia west on Walnut.

4.2 Left on 61 st st.; right at end 5.4 on Baltiniore.

5.9 Left at cemetery on Church Lawn; right after R.R., 6.3.

8.0 Right with trolley, Darby; left after bridge.

8.6 Toll gate. Pay 15-20c, show ticket at 4 gates.

15.0 Right with trolley on $3 \mathrm{~d}$ st., thru Chester, $\mathrm{Pa}$.

27.7 Left across $R$. R. and bridge; next right on Market.

28.1 Right at duPont Hotel, on 11th st., Wilmington.

28.8 Half left at fountain on Pennsylvania ave.

29.3 Left on Dupont st., right on W. 6th st., 29.6.

29.9 Left on Union st.; across R.R.'s at Elsmere 31.

32.8 Half left at 4-corners; cross R. R. 33.6.

33.9 Left around P. O., Marshalltown; right 34.1.

35.8 Sharp right beyond church; right at end 40.

41.6 Left before 2d R. R. Newark; left at end 47.5.

48.4 Right at C. H., Elkton, on Main st., thru Northeast 54.5 and Perryville 63.7. Cross long bridge (60c) and curve left; keep right of hotel Havre de Grace 64.5 on Union ave.

65.0 Right at cross street with good road.

69.5 Right at stone church, Aberdeen; curve leftright at park; thru Churchville 75.9 .

81.2 Left at small store, Bel Air; next right.

88.6 Right at 4-cor., Kingsville; thru Fullerton.

101.4 Half right at 5-corners with trolley, North ave.

103.1 Left with cross trolley on Charles st.

103.7 Hotel Belvedere, Baltimore at Chase st. 
Philadelphia, Pa., to New York, N. Y., 99.4 Miles

0.0 From City Hall, Philad., north on Broad.

4.6 Right on Blvd.; left fork at circle 8.

12.7 right fork at Bustleton; right 13.4.

27.4 Half left; across Delaware R. (20c.).

30.8 Right on State st., Trenton, N. J.

33.4 Right at school; right with trol.; 35.7.

39.2 Left Robbinsville; thru Hightstown 55.

62.4 1st right after R.R.; left on George 63.

68.2 Left over R.R. bridge Metuchen; right.

74.4 Left from underpass; under R. R. 81.

81.5 Left at C. H. Elizabeth; right fork 81.9.

82.1 Left fork Broad st., right on Clinton 85.8.

86.0 Left on High st., Newark, from trolley.

86.9 Right on market st., half left 88.6.

92.2 Left into park; right at circle 92.5.

92.8 Left on blvd. thru Jersey City.

98.1 Right at cem. on 3rd; left-right 98.3.

98.8 Jog left-sharp right, downhill to

99.4 Ferry for W. 42nd st., New York.

\section{HOTEL \\ EMPIRE}

Broadway at 63 rd Street NEW YORK CITY

"The Hotel of Easy Access" Room, use of bath $\$ 2.00$

Parlor, bedroom $\$ 4.00$
and bath,

Add to the above rates, 50c, for each additional person.

All Subway, Elevated, Surface and Bus Lines lead right to the door.

Beautiful Central Park-l block

Our Restaurant

is noted for its excellent food and moderate prices.

\section{Centrally} Cocated

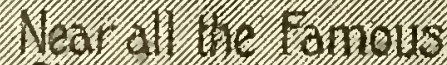

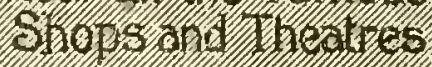

P. V. LAND

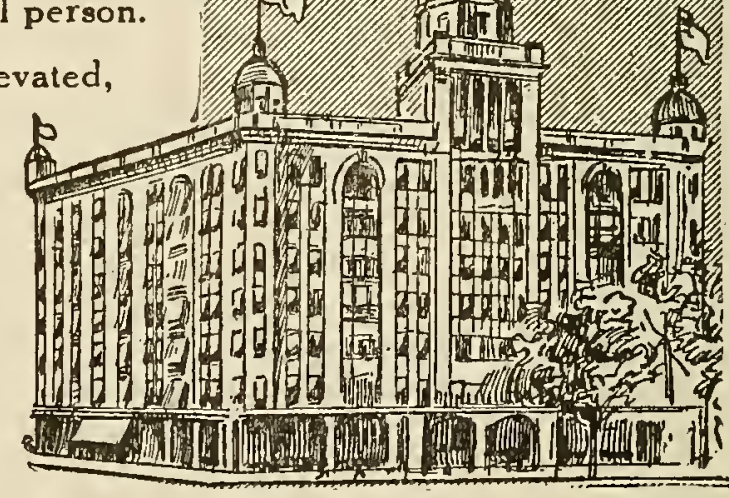

MANAGER

Kindly mention MOTOR TOURS when calling on or writing advertisers 


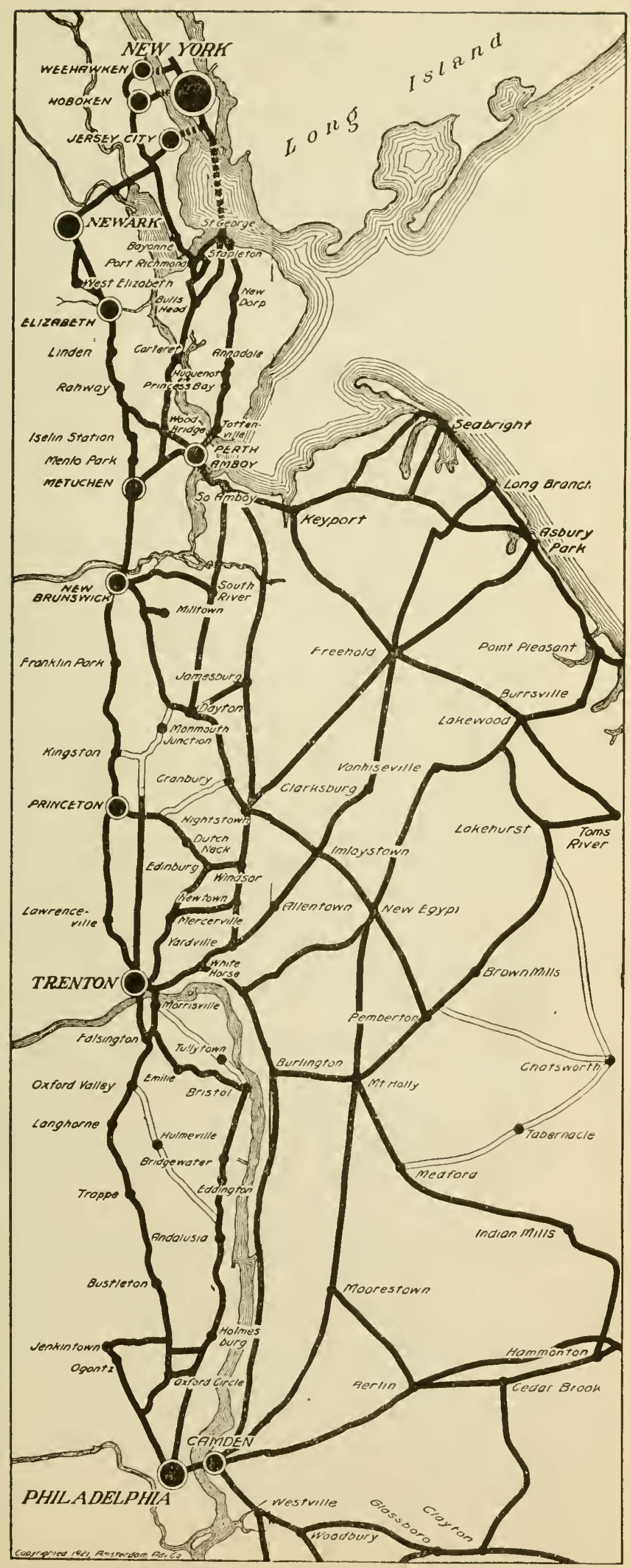


Washington to Baltimore, 37.7 Miles

0.0 From Treasury, Washington, northeast on $N$. $Y$. ave; bear right on I 0.4 ; bear right on Massachusetts 0.8 ; left on $\mathrm{H}$ st..

1.8 Half left at 6-corners with trolley.

5.4 Left at 3-corners, Bladensburg, leave trol.

34.7 Left at park on Monroe st. over R. R.

35.2 Right on Wilkins; next left on Fulton st.

36.0 Right on Mulberry st., leave trolley.

37.5 Left around cathedral on Charles st.

37.7 Baltimore, Washington monument.

Straight on Charles st. for Philadelphia.

\section{Baltimore to Philadelphia, 103.7 Miles}

0.0 From Hotel Belvedere, north on Charles st.

0.6 Right with cross trolley on North ave.

2.3 Half left with trolley on Bel Air rd.

15.1 Left at 4-corners, Kingsville; next right fork.

22.4 Left at Inn, Bel Air; next right 22.5.

34.2 Left at stone church, Aberdeen, after 2d R.R.

38.7 Left at 4-corners; Union ave., Havre de

Grace. Curve R. across Susquehanna bridge (60c.) thru Perryville 40; Northeast 49.2 .

55.3 Left at C. H., Elkton; right after R.R. 56.2.

57.2 Right fork with poles; left fork 57.9 .

62.1 Right at end Newark; left across R.R. 63.5.

68.2 Left past stone church; bear left 14.7.

69.8 R. around P. O., Marshalltown; bear L. 70.

70.9 Half right at 4-corners, meeting trolley.

73.9 Right on W. 6th st.; left on Dupont st., 74.1.

74.4 Right on Pennsylvania ave.; bear right on Delaware ave. 74.9 ; bear left at monument on 11 th st. to Hotel Dupont, Wilmington.

75.7 Left on Market; left across bridge, then right.

85.7 Right with trolley, Marcus Hook; next left.

63.5 Right on Albany st., New Brunswick.

88.8 Left on Morton ave., Chester with trolley under R.R.

Toll gate 90.1. Pay 15-20c for ticket.

95.7 Right at end across bridge; left at Darby, 95.8.

96.6 Right on Bailey rd.; left at end 97.5.

99.6 Right on Chestnut to Broad, Philadelphia

97.9 Right on Baltimore ave.; left on 61 st 98.4 .

Left on Broad for New York 103.7.

Baltimore to Washington, 37.7 Miles

0.0 From mon. Baltimore, so., on Charles.

0.2 Right at cathedral on W. Mulberry st.

1.7 Left at trolley on Fulton st.

2.4 Right on Wilkins; next left on Munroe.

3.0 Right at end on Columbia ave.

32.4 Right at 3-corners, Bladensburg join trol.

36.0 Half right at 6-corners on $\mathrm{H}$; bear right on Massachusetts 37.0 ; left on I st. 37.1 .

37.7 U. S. Treasury, Washington, D. C. 


\section{New York to Atlantic City, N. J., 117.6 M.}

0.0 Cross ferry to St. George, S. I.; land, curve left and right; then left, with trolley.

1.7 Right at Clifton sta. on Vanderbilt ave.

6.1 Left at tavern in fork; again left 7.1.

14.9 Right on Bentley st., Tottenville, to ferry; land at Perth Amboy; straight up Smith st.

15.4 Left at hotel on High st.; right at monument.

16.3 Left with trolley across bridge Raritan river.

17.7 Left with trolley; keep right 18.5, South Amboy.

18.7 Left on Stevens ave.; right at end 19.2.

23.6 Left at 4-corners; right at 3-corners 24 .

25.8 Right beyond road house; keep right.

27.9 Right at end, Main st., thru (Matawan) Beacon City.

39.2 Half left, South st., at City Hall, Freehold.

42.6 Right at Adelphia P. O. into Madison ave.

51.6 Straight thru Lakewood, across bridge; thru Tom's River 61.2; Barnegat 76.4; Manahawkin 81.6; Tuckerton 89.9 ; New Gretna 95.8.

96.4 Left at sign; left with poles 100.8; thru Absecon 110.1; follow Main st. across RR's at Pleasantville.

112.9 Left at sign on Verona ave. into boulevard.

117.6 Left on Atlantic ave., Atlantic City.

Atlantic City, N. J., to New York, 117.6 M.

0.0 From Atlantic ave., Atlantic City, northwest at park on Albany ave., becoming Verona ave.

4.7 Right on Main st., Pleasantville; thru Absecon 7.5; left fork Oceanville.

16.4 Right at end; right at end 21.2; thru New Gretna 22; Tuckerton 28; Manahawkin 36; Barnegat 40, Tom's River 56.4 .

66.0 Straight thru Lakewood on Madison ave,

41.0 Left at P. O., Adelphia, direct to Freehold.

78.4 Right at Court House; bear left at park 78.6; thru Marlboro 82.5 ; cross RR at Freneau.

89.7 Left at brick church beyond P. O., Matawan.

91.8 Left at end; bear left 93.6, and right 94.

98.4 Left beyond railroad bridge on Stevens ave.

98.9 Right with branch trolley Main st., South Amboy.

99.9 Right with trolley across bridge Raritan river.

101.3 Right at end; left at monument on High st.

102.2 Right at hotel on Smith st., Perth Amboy.

102.4 Cross ferry (50c.) to Tottenville, S. I.

102.7 Left at end on Amboy road; bear left 104.9.

110.5 Right at end, join trolley at New Dorp 111.5.

115.0. Right fork on Vanderbilt ave. from trolley.

115.9 Left at end, Bay st., St. George.

117.6 Right at ferry to Manhattan.

\section{Philadelphia to Atlantic City, 59.2 Miles}

0.0 Phila.-Camden ferry; east, Federal st.

0.7 Half-right Hadden ave.; right fork 2.6 ; thru Haddon Heights 6; Magnolia 8; Stratford 10; Berlin 16.

29.0 Right fork, Hammonton; thru Egg Harbor City 40, and Pomona 45.

51.6 Right at end, $\mathrm{Sisecon;} \mathrm{to} \mathrm{Pleasantville.}$

54.4 Left after RR, Verona ave., into boulevard.

59.2 Albany and Atlantic aves, Atlantic City.

\section{Wilkes-Barre to Scranton, Pa., 18 Miles}

0.0 From square, Wilkes-Barre, northwest on Market.

0.2 Right on River st. thru Pittston 7.7.

11.7 Left over bridge thru Holland 12.8.

17.1 Right with branch trolley on Jackson st.

17.5 Right Lackawanna ave. to Wyoming ave.

18.0 Scranton. 


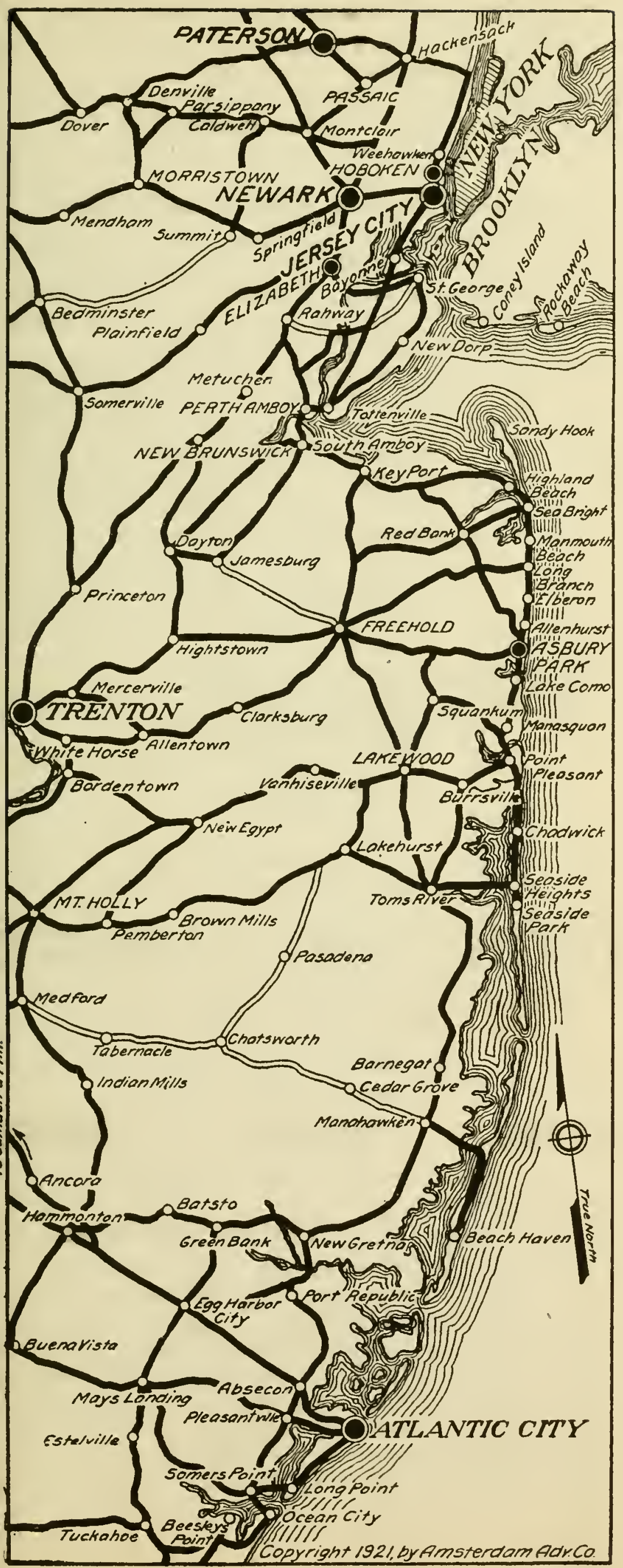




\section{Springfield to Hartford, 26.6 Miles}

0.0 From State st., Springfield, south on Main.

0.8 Right fork from trolley; left at end 1.1.

24.9 Right at $\mathrm{E}$. Hartford across stone bridge.

26.1 Left at end of bridge, curving left 26.4 .

26.6 Left at City Hall on Main st., Hartford.

\section{Hartford to New Haven, 36.7 Miles}

0.0 From City Hall, Hartford, south on Main st.

0.6 Right fork; right fork on Maple 1.0.

17.3 Right on Main st., Meriden; 3rd left on Cook.

20.3 Left under R.R.; at once right, thru Wallingford 23.4; and North Haven 28.4.

32.2 Half right at garage, into Middletown ave.

34.5 Right on Ferry st.; left after R.R. bridge.

35.2 Bear left on Bishop st. from trolley.

35.7 Left at trolley on Whitney; right fork 36.1.

36.7 Right on Chapel st. at green, New Haven.

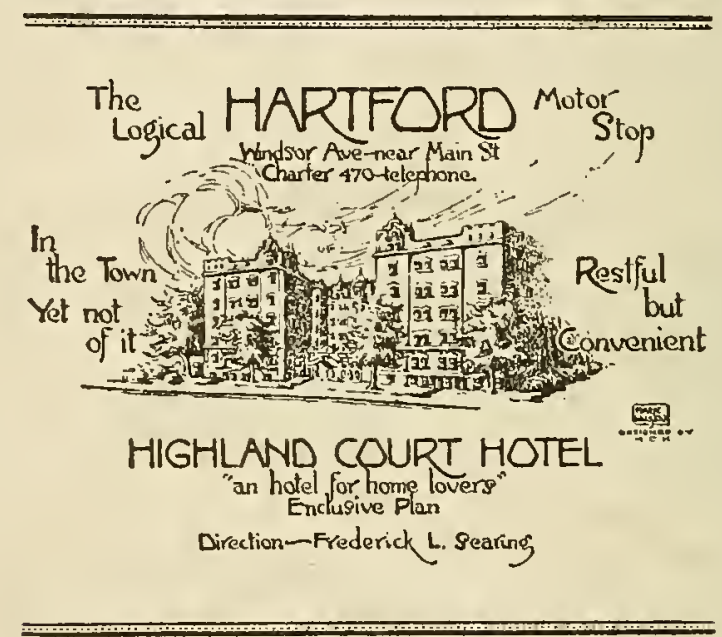

HIGHLAND GARAGE

Opposite Highland Court Hotel HARTFORD, CONN.

Day and Night Service

Competent Mechanics

STORAGE. WASHING

SUPPLIES

Telephones Charter, 5139-8575-6543

New Haven to Hartford 36.7 Miles

0.0 Chapel st., New Haven north on Temple.

1.0 Right on Bishop st.; bear left on State 1.5.

2.1 Right over R.R. bridge; next left.

4.5 Half left at garage; thru Wallingford 13.3.

16.3 Left under R.R.; sharp right thru Tracy.

18.2 Left fork Cook ave.; right on Main st., 19.2.

19.4 Left at station, Meriden, on Colony st.

20.3 Right fork across R.R.; left fork 22.9 .

36.7 Right at City Hall, Hartford, on Central. 
Albany, N. Y., to Pittsfield, Mass., 36.8 M.

0.0 From State st., Albany, south on Pearl, left across Hudson River; right, Rensselaer.

1.1 Left with trol. on Columbia; thru Schodack Cen., 7; Nassau 13; W. Lebanon 22; into New Lebanon.

26.1 Left at church; right at 3-corners over Taconic Mtn. thru Shaker Village 22; left fork 22.6 into Housatonic st.

36.9 Right on South st., Pittsfield.

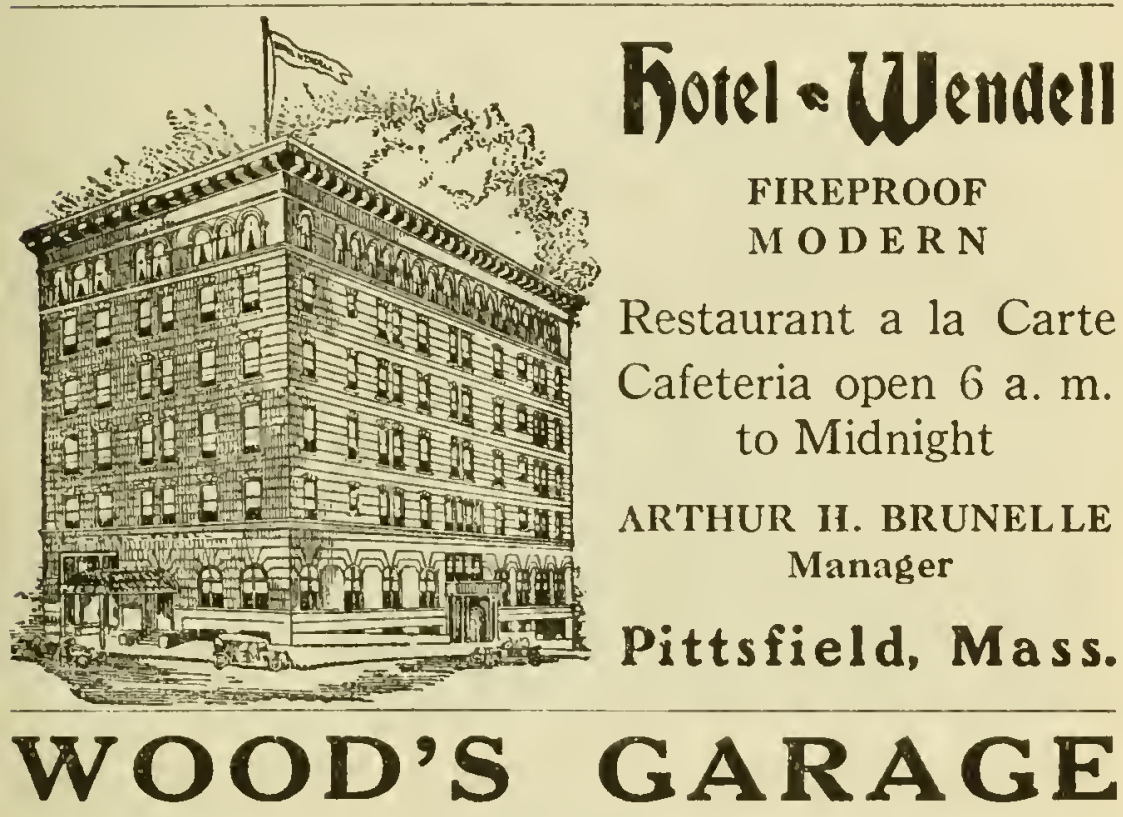

PITTSFIELD, MASS.

Near Wendell and Park Hotels

Pittsfield to Springfield, Mass., 56.3 Miles

0.0 From square, Pittsfield, south on South st.

6.6 Left at monument, Lenox, $\mathrm{R}$ at Lee, 10.6.

12.7 Right at hotel East Lee; thru W. Becket 54.5 and over Jacobs Ladder into Chester.

26.7 Right fork; thru Huntington.

38.0 Right at Russell ; into Franklin st., Westfield. 46.1 Right on Elm st.; left at fork on Main 83.1. 54.8 Left at W. Springfield over Connecticut Riv. 55.3 Half left coming into Main st. to R.R. arch Springfield 56.3.

\section{Hotel Bridgway}

Bridge St. and Broadway

SPRING F I L L , MASS.

EUROPEAN PLAN. Convenient for Social or Business Purposes. Quiet, homelike atmosphere, appealing particularly to motorists and ladies traveling alone

150 ROOM EACH WITH BATH

Restaurant a la Carte 
Springfield, to Pittsfield, Mass., 56.3 Miles

0.0 From Springfield, N. on Main st.; $L$ fork cross Connecticut River; $\mathbf{R}$ at W. Springfield 1.5.

10.2 Right at Westfield; left on Franklin st.

18.3 Left at Russell; thru Huntington 23.3 into Chester.

29.4 Left fork; over Jacobs Ladder; thru W. Becket 38.6.

43.6 $\mathrm{L}$ at hotel, E. Lee; L from trolley at Lee 45.7.

49.7 Right at monument, Lenox, to Housatonic st., Pittsfield.

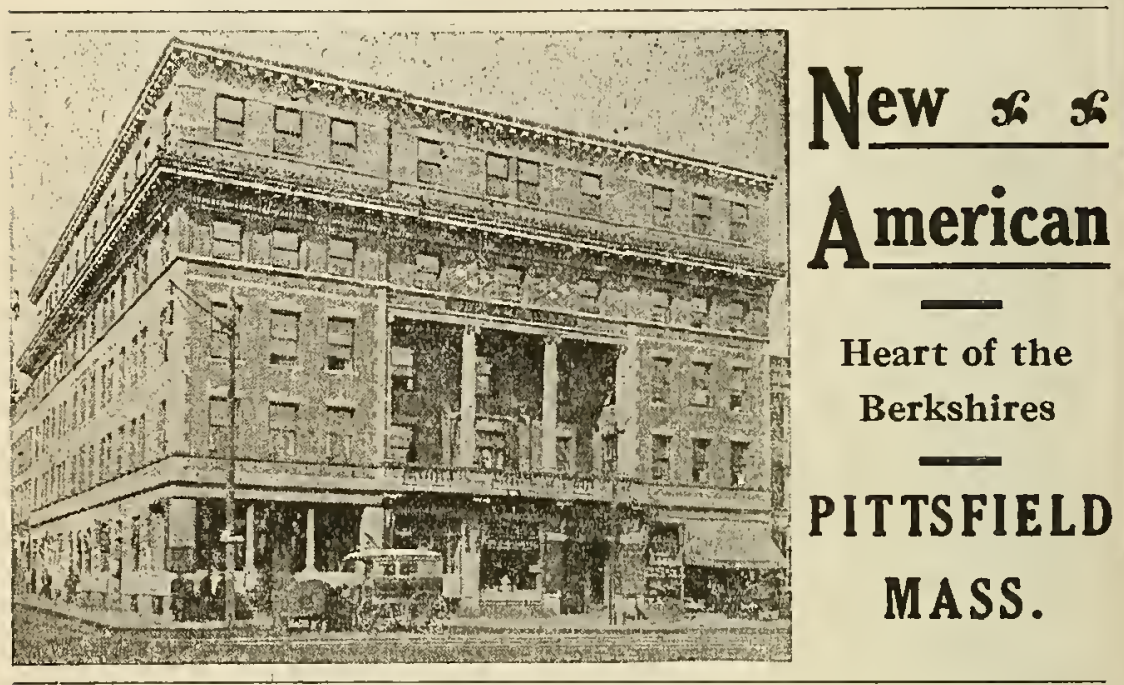

Pittsfield, Mass., to Albany, N. Y., 36.8 M.

0.0 From South st., Pittsfield, west on Housatonic st., half-left 0.2 , under R.R. and right 1.0 thru Shaker Village 4.7; New Lebanon 9.9.

10.7 Right at church thru W. Lebanon 14.7; Nassau 23.7 ; Schodack Center 29.0; E. Greenbush 31.7 to Rensselaer.

35.6 Right on Broadway; left across Hudson River bridge; right on Pearl to State st., Albany 36.8 .

\section{Sign of The Golden Robin}

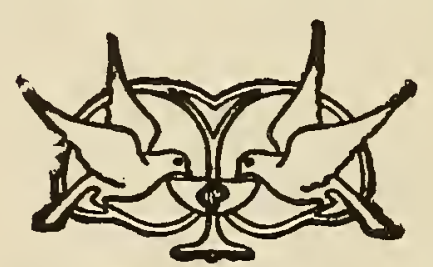

Home Cooked Lunches Served or Packed for your trip Delicious Candies

BLUE BIRD GIFT SHOP IN CONNEGTION TELEPHONE MAIN 3732 


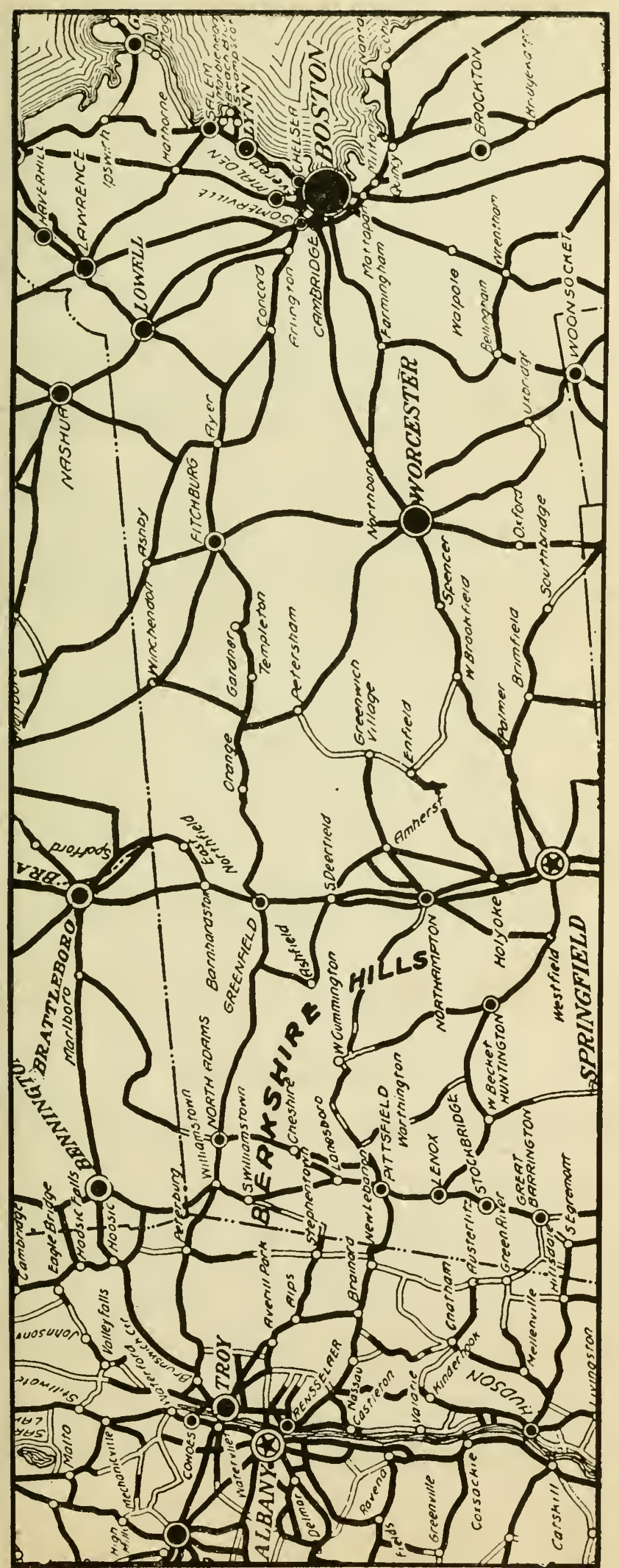


Boston to Worcester, Mass., 42.5 Miles :

0.0 From Massachusetts ave., Boston, west on Commonwealth.

0.3 Half left on Beacon; thru Brookline.

3.1 Right on Chestnut Hill ave.; next left on Commonwealth; thru. Auburndale 9.

10.3 Half right on Newton; right at end 12.1.

12.8 Left at end past church, Weston.

26.2 Left at trolley; right with tracks on Main.

27.0 Left fork at mon., Marlboro; right 27.7. Straight on at 27.7 for Southboro Arms.

37.2 Left fork with trolley at Shrewsbury.

42.1 Keep right at sta. on Front st. under R.R.

42.5 Left at City Hall, Worcester, on Main st.

\section{UNITED STATES GARAGE}

NEAR THE BIG HOTELS

LARGEST MOST MODERN FIREPROOF

130-160 Mechanic St. WORGESTER, MASS.

Worcester to Springfield, Mass., 50.5 Miles

0.0 From City Hall west on Main st.

2.0 Right fork at Webster sq.; keep right 2.2.

17.7 Right, next left with trolley, Brookfield.

20.1 Curve left thru W. Brookfield; left 21.5.

23.8 Over R.R. bridge, Warren; up steep hill.

35.2 Sharp left at end; right at end 35.4 Palmer.

36.5 Left fork with trolley; left under R.R. 40.6.

42.9 Left fork from trolley into State st.

50.5 Main st., Springfield (right for hotels).

\section{HOTEL WORTHY}

One of Springfield's Leading Hotels

Absolutely Fireproof

Commercial and Tourist

Not a New Hotel

But Old and Reliable

WORTHY INN - Manchester, Vt.

Same management

Wm. W. Benson, Manager

Boston to Falmouth, Mass., 80.6 Miles

0.0 From Massachusetts ave., Boston, west on Commonwealth avenue, curving left 0.1 at Collins monument. Follow main parkway along Jamaica pond.

4.7 Left at 3-corners, keep left 5.6 under railroad.

5.9 Curve right and left into Morton street.

7.5 Right on Blue Hill avenue, thru Mattapan 8.6.

Continued on page 87 
Springfield to Worcester, Mass.; 50.5 Miles

0.0 From Main st., Springfield, east on State.

1.4 Left fork at trough; right fork at cem. 2.9.

9.9 Left under R.R. at N. Wilbraham, next right. Steep descent into Warren 26.5.

15.1 Sharp left at hotel, Palmer; 2 d right 15.3.

29.0 Right at end; right fork W. Brookfield 30.

32.6 Right; next left with trolley, Brookfield.

50.5 Right on Front st., Worcester, at City Hall.

\section{"BE AT HOME AWAY FROM HOME"}

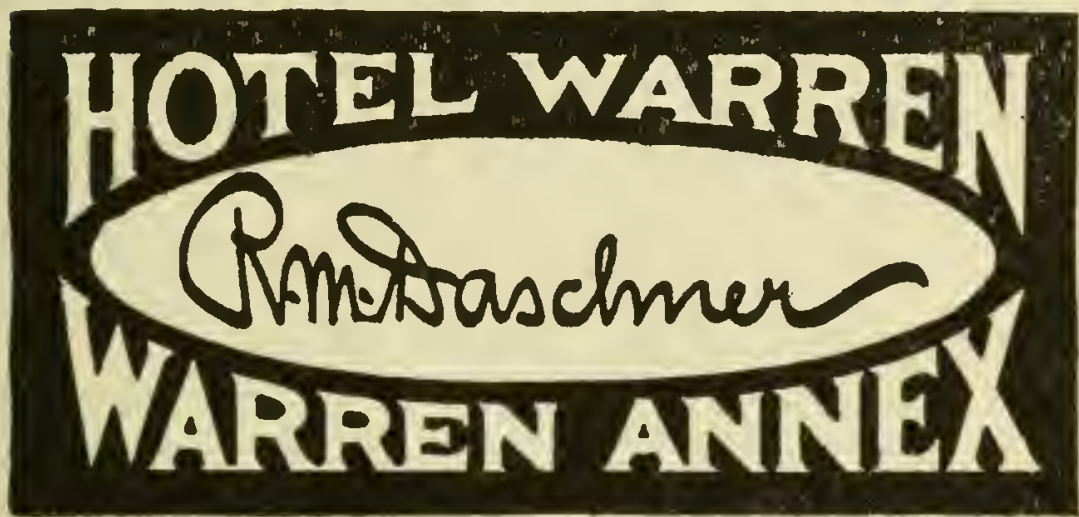

WORCESTER, MASS.

\section{UNITED STATES GARAGE}

NEAR THE BIG HOTELS

LARGEST MOST MODERN

FIREPROOF 130-160 Mechanic St.

WORCESTER, MASS.

Boston to Falmouth, 80 Miles

Continued from page 86

14.5 Left fork with trolley at Ponkapoag. (Right is via Mansfield). Go thru Stoughton 19.0.

27.7 Left; thru Cohasset 29.0 and W. Bridgewater 31 .

33.5 Right on Summer st., Bridgewater.

41.4 Left or. Center st., Middleboro, past P. O. 41.8 .

45.6 Right fork from trolley; join tracks 47.

53.2 Left at Tremont; right with trolley thru S. Wareham 55.0.

57.1 Left at Wareham; right after bridge 59.4.

59.7 Left after R.R.; thru Onset 61.

64.5 Right at Bourne over canal bridge; thru Monument Beach 67.

78.8 Left fork; half left on Main st., 80.3.

80.6 Falmouth, Mass.

"Stop, Look and Listen" at all railroad crossings.

Don't bring hardships on a motoring fraternity by your foolhardy acts.

Always remember that it is better to cause a delay than an accident. 
New York to Bridgeport, Conn., 58.2 Miles

0.0 From Pershing Square, north on Park ave., left on 57 th; right on Fifth ave., into Park.

1.3 Right fork at Mall; right out of park 3.9.

5.7 Right on 145 th st., across bridge.

6.4 Left at subway Kiosk on Mott ave. into Wilson Parkway.

9.8 Right on Fordham rd.; thru Bronx Park.

13.8 Left on shore road; into New Rochelle.

18.6 Right on Main st.; thru Larchmont 20.3.

25.5 Right fork at flagpole, Rye.

27.2 Left at trolley, Portchester, keep right 27.3; thru Greenwich 30.1; Stamford 35.2.

35.5 Left fork at park; under R.R. at Darien 39.7.

44.0 Left around green, Norwalk; right 44.2 with trolley thru Westport 47.2; under R.R. at Southport 51.2 into Fairfield.

52.7 Right and next left into Bridgeport 58.2.

Bridgeport to New Haven, Conn., 17.4 M.

0.0 From Main st., east on Fairfield ave., Bridgeport; cross 2 iron bridges and left fork 0.9 .

8.1 Left at end of common, Milford, under R.R.

16.8 Jog right-left at end of Davenport ave.

17.0 Right, next L College st., New Haven 17.4.

New Haven, Conn., to New London, 52.6 M.

0.0 From New Haven, E. on Chapel; $\mathrm{R} 1.9$.

2.2 Right after bridge; left at trough 2.9 ; thru $E$. Haven 4.5, Branford 8; York st., Guilford.

15.5 Right at end; next left; next right; next left at Green, thru Madison 21, and Clinton 24.5; Westbrook 29.

33. Left at inn, Saybrook; keep right over Connecticut River.

37.2 Left at end; thru E. Lyne 45.7 into New London 52.6.

\section{New London to Providence, 73.5 Miles}

0.0 From mon. east to Ferry for Groton (18.33c.)

0.1 Right on Thames; L fork; L under R.R. 2.0.

2.5 Right at end; $\mathrm{R}$ at end $4.5 ; \mathrm{L}$ at end 5.3 .

8.0 Right with trolley, cross bridge into Mystic.

8.5 Right follow trolley; left at Stonington 12.7 .

17.8 Left at school, Westerly; $\mathrm{R} 8.0$; right 18.4 .

$39.1 \mathrm{R}$ beyond R.R. Wakefield; R 39.5; L 40.2 .

41.8 Left at end Narragansett Pier; thru Hamilton 51.3.

53.7 Left at end, Wickford; right at end 54.3; thru E. Greenwich 61.3.

63.5 Right at Appenaug Hotel; left at sta.; left 67.8 into Elmwood ave. and Weybosset.

73.4 Left on Dorrance st. to City Hall, Providence 73.5 .

Providence to Boston, page 93 


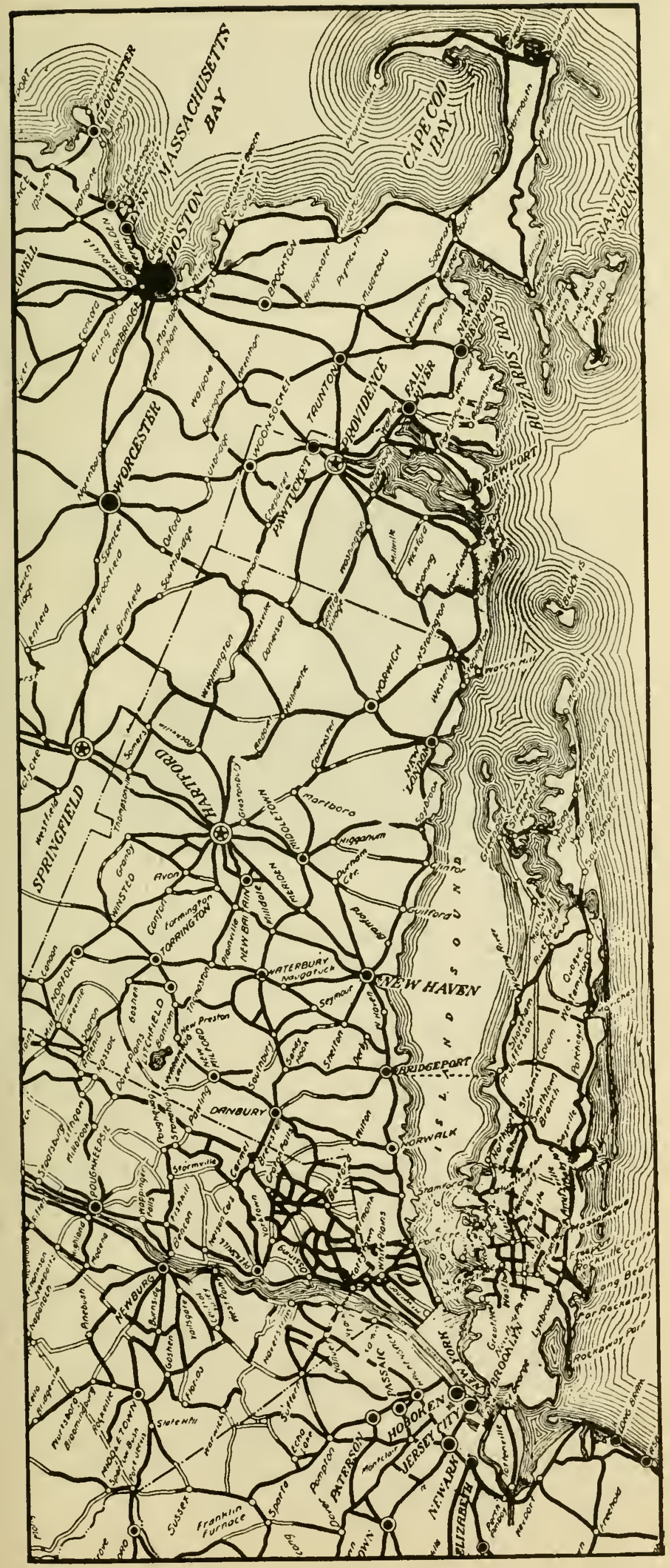


New Haven to Bridgeport, Conn., 17.4 M.

0.0 From Hotel Taft, New Haven; south on College st. Jog right-left at end on Broad.

0.3 Jog right, half-left on Davenport ave.

9.0 Under R.R., at once right thru Milford 9.3.

13.4 Left at 3-corners, Stratford; right 13.8.

15.0 Right fork, Connecticut ave. Cross bridge.

17.1 Bridgeport. Follow trolley across Main st.

\section{The Strratfield (IIRProor)}

\section{BRIDGEPORT, CONNECTICUT}

290 Rooms with Bath

F. A. CANTWELL, - - - Manager

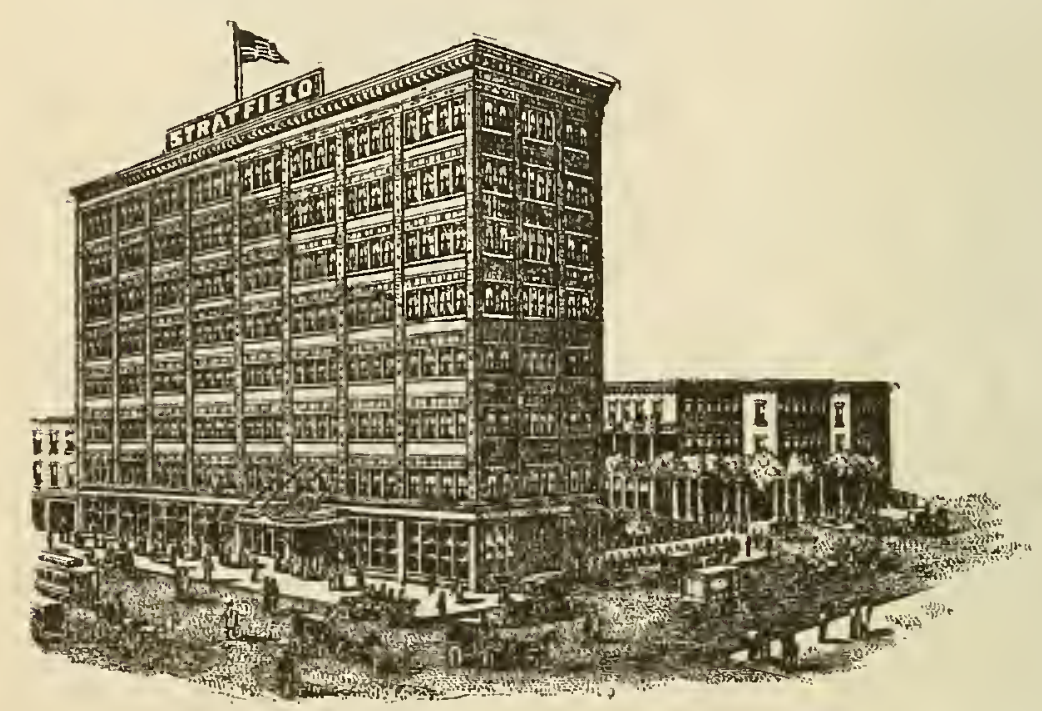

Superior accommodations for motorists

Spacious sun parlors and porches

Surrounded gardens and well-kept lawns

Bridgeport to New York, 58.2 Miles

0.0 From Main st., west on Fairfield ave.

4.1 Right and next left with trolley, Fairfield; under R.R. at Southport 5.7, thru Westport.

12.7 Left at end; right around green, Norwalk.

13.7 Right fork at water trough, leave trolley under R.R. at Darien 17.2; thru Noroton 18.6.

21.7 Left fork at park by city hall, Stamford; thru Greenwich 26.8 to Portchester.

29.7 Right under R.R.; right fork Rye 31.5 ; thru Mamaroneck 34.9, Larchmont 36.6.

38.3 Left at hotel on Echo ave., New Rochelle.

43.1 Right on Pelham parkway, thru Bronx park.

47.1 Left on Wilson parkway; left at mon., 49.8.

50.5 Right at subway kiosk, on 149th st.

51.2 Left on Seventh ave., left thru Central Park.

56.1 Left on 57th st.; right on Park ave. to Pershing Square 58.2. 
Providence to New London, Conn.

\section{Che Inohican botel}

NEW LONDON, CONN.

Overlooking Long Island Sound

European Plan Unsurpassed Equipment

400 Rooms with Bath

MIDWAY NEW YORK TO BOSTON

\section{The Garage De Cuxe of Fimerica}

\section{Monte Cristo Garage}

JAMES P. SULLIVAN, Proprietor

New London, Conn.

Opposite Mohican Hotel

\section{Capacity 268 Cars}

New London, Ct., to New Haven, Ct., 52.6 MI

0.0 From monument run west on Bank st.; right fork 2.0 .

5.4 $\mathrm{R}$ from trolley over Conn. River toll bridge. 19.5 Right around inn at Saybrook; thru Westbrook 23.6 and Clinton 30 .

36.6 Right at Guildford; left at end; next right; next left; then right fork; thru Branford 44.6.

49.7 Right at trough; left fork; left fork 50.1.

50.4 Left over bridge; left on Chapel st., 50.7.

52.6 New Haven, the Green on right.

\section{HOTEL GARDE}

Opposite Union Station

NEW HAVEN - $\quad-\quad-\quad-$ CONN.

On direct motor route-New York to Boston

Leading hotel for motorists
A large modern hotel at moderate prices 
Boston to Greenfield, Mass., 97.5 Miles

0.0 From Commonwealth, north on Massachusetts ave.

2.7 Right at Harvard square; curve left thru Arlington 6.

11.5 Left fork at Lexington; right-left at Concord 18.

12.0 Right fork Elm st., thru North Acton 25, past lake.

27.8 Left fork Littleton Common; right fork 28.5.

34.4 Right at Ayer; left 35.2; thru Lumenburg 43.

47.5 Left fork Fitchburg; right at end Westminster 54.

58.1 Right fork South Gardner; left on Main, Gardner; left 60 .

64.9 Left at Baldwinsville, thru Athol 74 ; Orange 79.

89.9 Left across railroad and bridge; right at Millers Falls.

93.2 Right; left at end, Turners Falls 95; right over bridge, then left upgrade into High st. to Main st., Greenfield 97.5.

\section{Greenfield to Albany, N. Y., 84.1 Miles}

0.0 From High st., Greenfield, west on Main; right after bridge; go over mountain; thru Shelburne 5.4.

8.8 Left at Shelburne Falls across Deerfield river.

9.4 Right at end on State st.; right over railroad 9.8; go over Hoosac Mountain, reaching Whitcomb summit 29; descent into Union st.

36.1 Left at end on Eagle; right on Main st., North Adams; left fork from trolley 37.5 .

41.2 Main and Water sts., Williamstown, Greylock Hotel.

41.7 Right on North st.; left at end 42.4.

46.9 Keep right across bridge and take left fork South Pownal.

49.6 Left fork at postoffice, North Pownal.

53.3 Right at North Petersburg; left at end 55.7 ; thru Potters Hill 59.6; Boyntonville 61.7; Pittstown 64; and Raymertown 67.

73.0 Left fork Center Brunswick; into Hoosick st.

76.8 Left on Fifth ave., Troy.

77.4 Right on Broadway; left at monument 77.6.

77.7 Right over Hudson river bridge; left on Broadway, Watervliet 78 .

84.1 Broadway at State st., Albany.

\section{Greenfield to Boston, Mass., 97.5 Miles}

0.0 From Main st., Greenfield, north on High; right 1.5.

2.6 Left at end after bridge; next right, Turners Falls.

7.1 Left down grade into Millers Falls; left, cross bridge. then right; thru Erving 14; and Orange 19.

23.5 Right fork on School st., Athol; left fork 24.5 .

33.1 Right at park, Baldwinsville; thru Otter River 34.

37.8 Right at West Gardner; right at end 38.8.

38.9 Right over railroad bridge; into Westminster 43.

43.4 Left, from trolley; thru Crockerville to Fitchburg.

50.6 Left on Lumenburg st.; thru Lumenburg 54.6; Ayer 63.

63.1 Right over railroad, next left; right fork Littleton Common 69.7; thru North Acton 73; left fork 76.7 into Concord.

79.3 Bear right and left thru Lexington 86; Arlington 91.

94.5 Right at Common; left at Harvard square, Cambridge, to Commonwealth ave., Boston, 97.5.

Hold the speed of your car to fifteen miles per hour and ten miles in the congested district, and you will get there more quickly, safely and pleasantly and at less expense.

In case of accident, stop at once, give your assistance and the name of every witness. 
Providence to Boston, Mass., 43.6 Miles

0.0 From P. O. Providence, east across plaza and across Benefit st., into Waterman.

0.5 Left on Thayer; right on Main; left on Broadway Pawtucket; thru North Attleboro 12, Wrentham 19, Walpole 25.4, Dedham 34.

34.6 Half $\mathrm{R}$ on Bridge; into Parkway thru Fens. 43.4 Left; right at Collins Mon. to Massachusetts ave., Boston 43.6 .

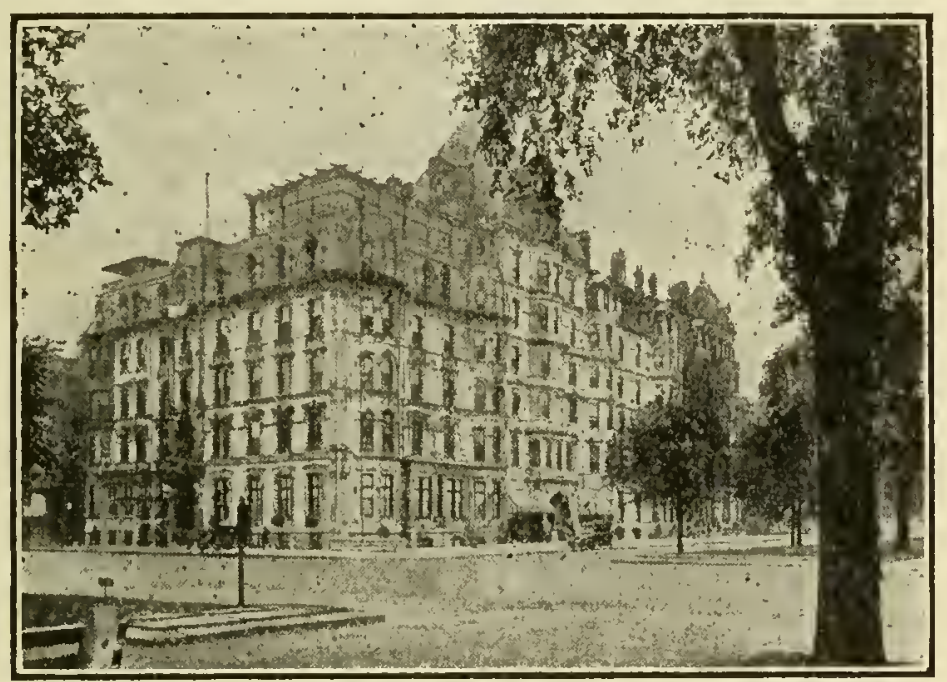

\section{HOTEL VENDOME}

Commonwealth Ave. at Dartmouth St. BOSTON, MASSAGHUSETTS

The most superbly situated Hotel in Boston, overlooking the beautiful Back Bay District, Commonwealth Avenue, the Public Garden, and the New Charles River Esplanade. American and European Plan.

The Vendome is much in favor with touring automobile parties who appreciate $a$ cool, quiet and refreshing night's rest after the tiring journey of the day

C. H. GREenleaf CO., Prop.

EVERETT B. RICH, Managing Director F. K. PIERCE, Asso. Mgr. Affillated Resort: PROFILE HOUSE, White Mountains, N. H.

Falmouth to Boston, Mass., 80.6 Miles

0.0 West on Main st., Falmouth; right at end 0.3, thru Monument Beach 13.6.

16.1 Left at end after canal bridge, Bourne; thru Onset 19.5 .

20.9 Right across R.R.; R at end, Wareham 23.5.

25.6 Right fork, South Wareham; left next right at Tremont 27.4.

39.2 Right on Everett st., Middleboro.

47.1 Left on Main st., Bridgewater; thru Cohasset 51.6.

53.0 Right on main road thru Stoughton 62.5; Ponkapoag 66.

68.4 Right fork leave trolley, keep right through Mattapan 72 on Blue Hill avenue.

73.1 Left at real estate office on Morton street.

74.7 Right and left curve under "L" and railroad.

76.0 Right at 3-corners; follow main parkway.

80.5 Right at monument on Commonwealth ave.

80.6 Massachusetts ave., Boston, Mass. 
Albany to Greenfield, Mass., 84.1 Miles

0.0 From State st., Albany, north on Broadway.

6.1 Right over Hudson river bridge; next left on River st.

6.5 Right at monument, Troy, on Broadway.

6.7 Left on Fifth ave.; right on Hoosick st. 7.3 thru Center Brunswick 11; Raymertown 17; Pittstown 20 ; Boyntonville 22.4, and Potters Hill 24.5.

28.3 Right fork; left at North Petersburg 30.7.

34.3 Right fork, North Pownal; L at church Pownal 37.5. 42.5 Left around Greylock, Williamstown, on Main st.

47.9 Left on Eagle st., North Adams, with trolley.

48.0 Right on Union; right from trolley 48.7; up Hoosac Mountain, reach summit 55.2.

73.9 Left after railroad bridge; left 74.7 across bridge Deerfield river at Shelburne Falls.

74.3 Right at end, thru Shelburne 78.7, over mountain.

83.2 Left over bridge into Main st.

84.1 Main and High sts, Greenfield.

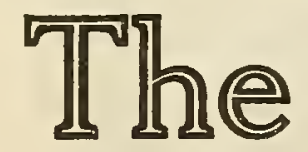

Berkshire Hills

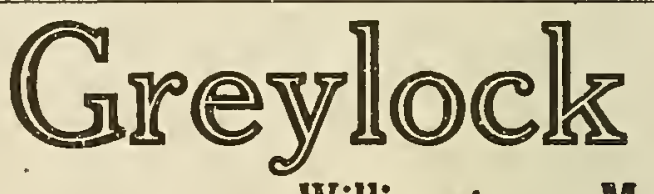

Williamstown, Mass.

On The Mohawk Trail

The Most Wonderful Scenic Motor Highway in the East Rooms en Suite. 110 Baths. Swimming Pool. The favorite stopping place for Tourists. Our own Fireproof Garage Send for Booklet HENRY N. TEAGUE, Lessee

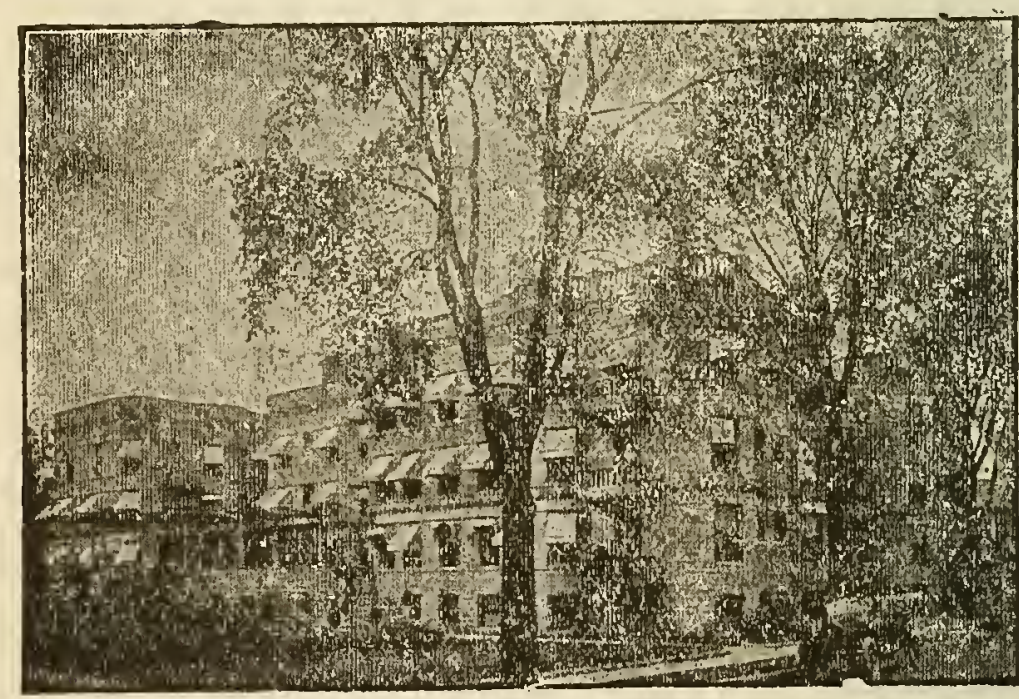

\section{THE WELDON HOTEL}

"JUST LIKE HOME"

GREENFIELD, MASS.

On the direct route to the Mohawk Trail. 200 Rooms. 150 with Bath. EUROPEAN PLAN

$\$ 1.50$ to $\$ 3.50$ per day. A la Carte Service all hours. Golfing and Tennis N. A. CAMPBELL - - Manager

WELDON GARAGE,InC. ${ }_{\text {PLACE TO STOP }}$

One of the Most Up-to-Date Garages in the State

Open Day or Night

Storage for 150 Cars Repairing of any kind and Vulcanizing

We sell tires, tubes and accessories

GEO. W. WILCOX, Pres.

Greenfield, Mass. 
Boston, Mass., to Providence, R. I., 43.6 M.

0.0 From Massachusetts ave., Boston, S. W. on Commonwealth; $\mathrm{L}$ at Collins Mon.; $\mathrm{R}$ on Audubon Rd.

4.1 Right on Center st. ; right on Spring; half left 9.1 on Ames; thru Dedham 10; $R$ fork 12.5 .

18.4 Left and right at Walpole; thru Wrentham 25. Follow trolley to North Attleboro 32.

39.7 R on Main, L on East st., Pawtucket, R. I.

43.3 Right at Brown University on Waterman; right-left 43.6 across Benefit st., to P. O., Providence.

\section{HOTEL ALLEN}

11 Greene Street

Providence, R. I.

All outside rooms with every modern convenience.

Centrally located, only four minutes walk to stores and theatres.

Tourists will find this hotel the coolest place in the city.

"A spacious yard shaded by trees

Where guests may sit and enjoy the breeze"

To reach us follow Westminister or Washington Street to Greene.

Rates $\$ 1.50 \mathrm{Up}$

With Bath $\$ 2.50$ up

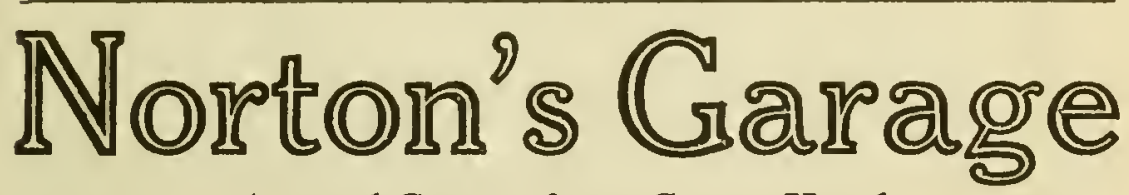

Around Corner from Crown Hotel PROVIDENGE, R. I.

\section{Storage}

Supplies

Washing

Expert Mechanical Service Two Hundred Car Capacity OPEN DAY AND NIGHT

Annox: NARRAGANSETT HOTEL GARAGE, Opposite Narragansett Hotel

Providence to New London, 73.5 Miles

0.0 From P. O. Providence, run west.

0.1 Left on Dorrance st.; $\mathrm{R}$ on Weybosset; $\mathrm{L}$ fork Broad; $\mathrm{R}$ fork Elmwood ave.; $\mathrm{L}$ fork 2.2 .

9.4 Right at sta. Apponaug; left at hotel 9.9, thru E. Greenwich 12.3.

19.2 Left into Wickford; right 19.7; thru Saunderstown 25.1.

31.8 $\mathrm{R}$ at Narragansett Pier; $\mathrm{R}$ at end; $\mathrm{L} 33.1$.

34.5 Left across R.R. at Wakefield; thru Charlestown 44.1 .

55.2 Left at water trough; left 55.7 ; next right on Broad st., Westerly.

56.4 Left fork; right with trolley Stonington 60.6. Follow trolley thru Mystic 65.3, and West Mystic 66.3.

67.5 Right; left at barn 69.2 ; left under R.R. 70.1; right at end.

73.5 Left at Groton to ferry for New London. 
Worcester to Boston, Mass., 42.5 Miles

0.0 From City Hall south on Front st.

0.4 Left fork at Union sta. on Shrewsbury st.

3.4 Half left with trol. thru Shrewsbury 5.1.

10.2 Cross R.R. at Northboro, to Marlboro.

16.0 Left fork at tree, right at trough 16.3.

29.6 Right fork at church, Weston; at once right on School st., bear right on Wellesley.

30.4 Left on Newton st.; half left on Commonwealth 32.2, thru Auburndale 33.7.

39.2 Right fork, Chestnut Hill ave.; next left 39.4 on Beacon Blvd.; half right on Commonwealth 42.2.

42.5 Massachusetts ave., Boston.

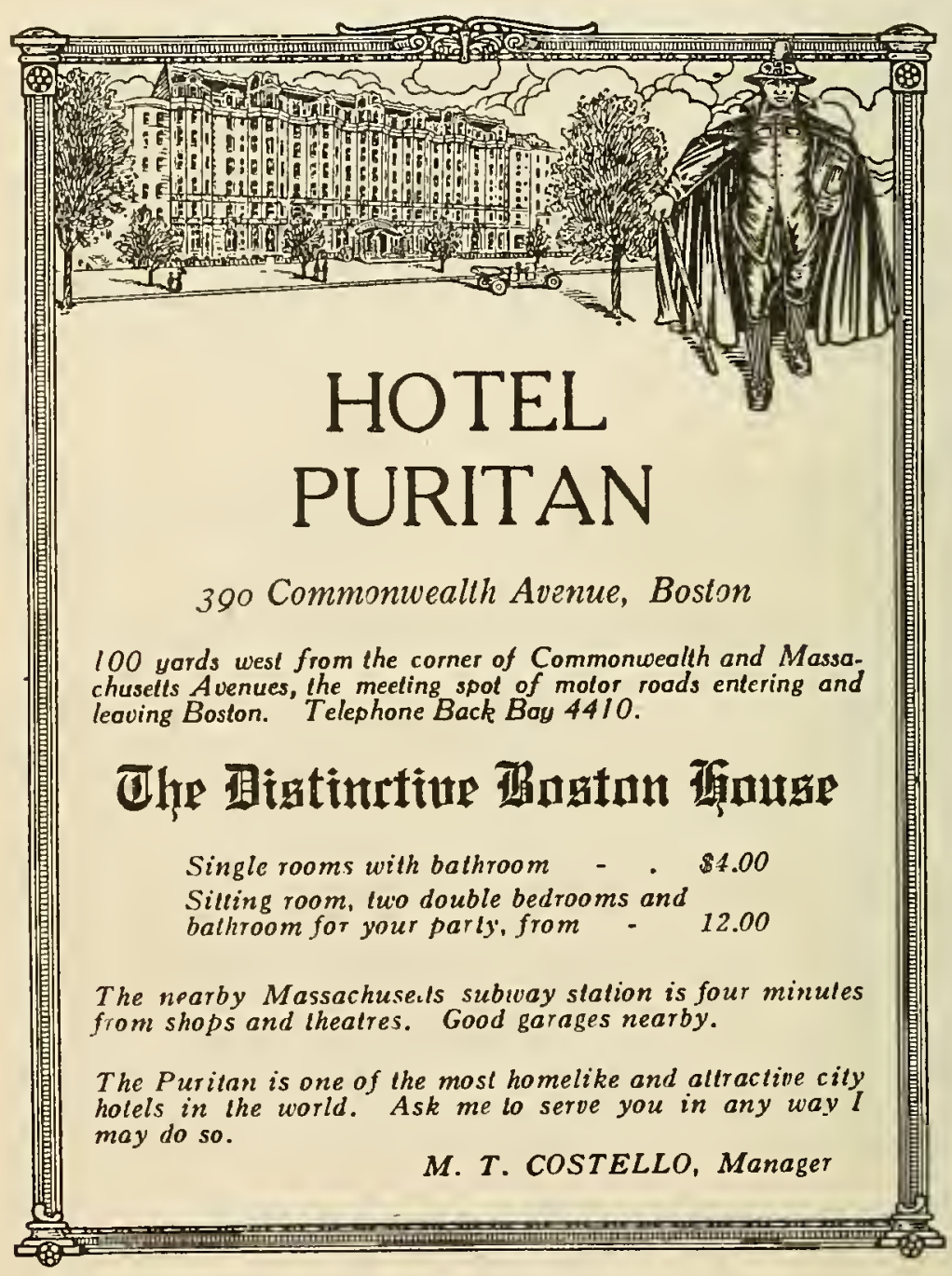

AUTOMOBILE CLUBS

5-Have promoted and enacted into law, Uniform Traffic Regulations, the Anti-Glare Headlight Law, and devoted their best efforts to the creation of the State Police.

6 -Have saved the motorists of the State in the last three years alone, SEVEN MILLION DOLLARS.

7- Have made it possible to operate an automobile in New York State on better roads and at less cost than any other State in the Union.

8-Have kept the motorists informed of all measures introduced at Albany, affecting their interests, kept them informed as to road conditions, and all other matters of interest to them. 


\section{Albany, N. Y., to Rutland, Vt., 105.5 Miles}

0.0 From Pearl st., Albany, north on State.

0.2 Right-left at Capitol on Washington ave.

0.8 Right fork, at once right on boulevard.

1.5 Left at triangle; curve right over viaduct.

2.3 Left at four corners; thru Lathams 7.3.

8.7 Right; left fork 9.9 ; right 10.4 .

11.9 Left, Congress st.; right at end; next left.

12.2 Right, Ontario st.; left, Saratoga st. 12.4; cross Mohawk river; right after canal.

14.2 Left, Third st., Waterford, to Mechanicville 23.3; thru Stillwater 26.5; Bemis Heights 29.

29.4 Left fork; right fork 30.3; thru Battlefield.

32.9 Right at end; thru Quaker Springs 34.5.

37.8 Right at end; thru Schuylerville 40.8.

42.9 Right over Hudson river, Northumberland; left fork 45.6.

46.9 Right over canal; right at cross street with trolley on Broadway; thru Fort Edward into Hudson Falls 56.7.

50.7 Right at monument in fork, shortly leave trolley; go thru Kingsbury 61.6; Comstock 70.

77.5 Right over bridge, Whitehall; curve right.

78.1 Left fork, Poultney st.; right fork 79.1.

81.8 Left at four corners; right 84.2 ; left at end 84.9.

87.9 Left at end; right fork Fair Haven 89.1; thru Left at end; right fork Fair Haven 89.1
Castleton Corners 91.5 ; West Rutland 100.3.

104.3 Right with trolley, Grove st.; left at station.

105.5 Rutland, Center st. at Wales.

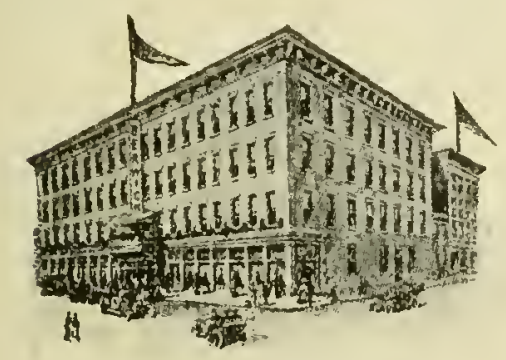

In the Heart of the Green Mountains

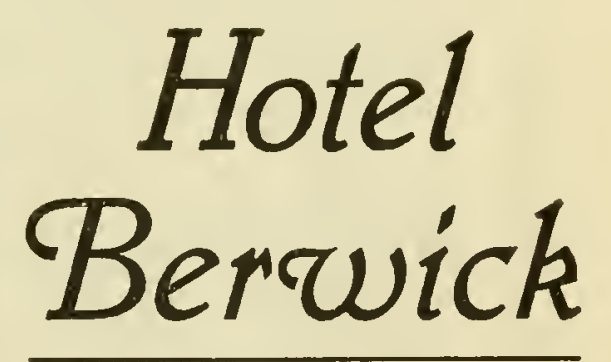

Rutland, Vermont

A Country Hotel Modernly Equipped, whose aim is to render its Service with Simpiicity yet with Adequacy

Especially known for the Excellence of its Foods

Rutland to Burlington, Vt., 69.3 Miles

0.0 From Wales st., Rutland, east on Center.

0.2 Left at trolley, on Main st.; trolley leaves 1.

2.2 Left fork; right fork 4.2.

7.4 Right thru covered bridge into Pittsford.

8.7 Curve right at bandstand; left fork 8.9.

14.7 Left with travel; right fork 15.3.

16.1 Left at monument, Brandon, past inn.

16.2 Right at end, next left; right at monument.

23.4 Right at Salisbury; left forks 23.8-25.4 past Lake Dunmore. Scenic oRute. Same distance to Burlington.

26.9 Right at end; keep left; curve right on Court st.

33.9 Left; next right, Pleasant st., Middlebury.

45.8 Left at end; half right on Green st. 46.5.

46.9 Right, Main st., Vergennes; curve left 47.5.

47.8 Left over covered bridge; thru Ferrisburg 49.5.

57.9 Left; thru Shelburne 62.4; left 63.1.

68.5 Bear left, St. Paul st. from trolley.

69.3 Burlington, Vt., St. Paul and Main sts.

\section{OIL THE SPRINGS}

Car will ride a hundred per cent better if leaves of springs are occasionally oiled and the spring clips tightened. . Will also save you money. 
Burlington to White Mountains, $112.1 \mathrm{M}$.

0.0 From St. Paul st. east on Main st., thru Williston 7.8 ; curve right after railroad bridge 10.9 ; thru Richmond 12.9 .

25.0 Right over covered bridge, Waterbury 27.

27.5 Right thru covered bridge; under railroad 32 .

32.4 Left at end, Middlesex, into Montpelier 38.6.

38.9 Right at end; next left with trolley 39.

41.0 Left across railroad; next right; left at end 45.4, thru Plainfield 49.3 ; into West Danville.

66.4 Right; thru Danville, into Western ave.

75.8 Left on Main st., St. Johnsbury.

76.0 Right down grade on Eastern ave.

76.3 Sharp left at end of avenue on Railroad st.

76.4 Next right on Portland st.; cross railroads.

79.3 Right at three corners beyond second covered bridge.

79.9 Left at three corners; left at three corners 85.8.

89.0 Right at four corners beyond school, Waterford.

89.4 Left at end of road beyond toll bridge (25c.)

94.0 Right at end of road; left fork 95.3.

95.3 Right at monument, Littleton; left at hospital 19.7.

101.4 Right at Bethlehem Junction station.

106.8 Right at hotel; right 32.8 .

112.1 Bretton Woods at railroad station.

White Mts., N. H., to Portland, Me., 109.9 M.

0.0 Bretton Woods station; descend steep grade thru Crawford Notch; cross railroad at Bemis 11.8; thru Bartlett 18 .

24.1 Right fork at Glen thru Intervale 27.6.

29.6 North Conway at P. O.; straight on.

32.3 Left fork; left at end 34.9 ; thru Center Conway 35.4 and Fryburg. 40.5 .

40.6 Right at sign; bear left 40.9 ; thru East Fryburg 46.2.

43.5 Right at end; right fork, Bridgton 45.4.

46.0 Half right at four corners; left fork 49.8 .

54.3 Left at three corners over bridge, Naples.

65.6 Left at sign; right 66.3; thru Cook's Mills 37.9.

72.1 Left fork, Webbs Mills; right fork 77.9.

82.0 Left at end; uphill to Poland Spring 83.1.

85.6 Right fork thru Sabbathday Lake 87.1; Dry Mills 90.7.

93.2 Right at four corners, Gray; half left at monument 93.3.

106.3 Right at trolley, Allen's Corners, on Allen ave.; bear left at end; cross railroad at Woodfords 108.3.

109.9 Forest ave. at Congress st., Portland.

\section{Portland, Me., to White Mts., N. H. 109.9 M.}

0.0 From Congress st., Portland, N. W. on Forest ave.

2.7 Right fork after railroad on Allen ave.

3.6 Left at Allen's Corners; at once right fork.

16.7 Left at Gray; right fork, Dry Mills 19.3.

22.8 Right fork, Sabbathday Lake; keep right 24.9.

26.8 Straight on at Poland Spring down grade.

27.9 Right; thru Poland 30; left fork 30.6.

38.3 Right fork; thru Cook's Mills 42.4.

45.6 Right after bridge, Naples, along lake.

53.9 Half left; left fork 54.5 to Bridgton.

54.6 Right fork at monument; left 56.4 ; thru East Fryburg 63.7 .

69.3 Left at end; thru Fryburg 70; Center Conway 74.5.

75.4 Right at sign; right at three corners 77.6.

80.3 North Conway at P. O. ; straight on thru Intervale 82.3.

85.9 Left fork; thru Bartlett 12; Bemis 18; ascend steep grade thru Crawford Notch 106.

109.9 Bretton Woods station. 
White Mts, to Burlington, Vt., 112.1 Miles

0.0 From Bretton Woods station, run north.

5.3 Left, right at Tivin Mountain Hotel.

10.6 Left across bridge at Bethlehem Junction.

16.7 Left at monmment, Littleton; right fork 12.2 .

19.2 Right fork; left 21.5 ; right; cross bridge 16.6.

23.0 Left at four corners, Waterford; right fork 20.2.

35.6 Left at end Railroad st.; right, Eastern ave. 35.7.

36.0 Left on Main st., St. Johnsbury.

36.2 Right fork, Western ave.; left fork 36.9.

46.2 Left at West Danville; thru Plainfield 62.8.

66.7 Right before covered bridge; right 73.1.

73.2 Left on State st., Montpelier, past Capitol.

79.7 Right at Middlesex; left after underpass.

84.6 Left at end thru Waterbury 85; left at end 87.2.

101.2 Left over railroad bridge; thru Williston 104.2.

112.1 Burlington, left on St. Paul st. for Rutland.

\section{Burlington to Rutland, Vt., 69.3 Miles}

0.0 From Main st., Burlington, south on St. Paul.

6.2 Right at end; thru Shelburne 7.

11.4 Right; left 11.8; thru Ferrisburg 19.8.

21.5 Right under railroad into Main st., Vergennes.

22.4 Left, Green st.; half left at four corners 22.6.

23.3 Right fork; cross RR at New Haven Junction 27.3.

35.2 Left fork at park, Middlebury, Pleasant st.

35.8 Left on Washington st.; next right, Court st.

42.4 Left; pass Lake Dunmore; left Salisbury 45.9

Scenic route via Lake Dunmore same as to Rutland.

52.7 Left at monument; right-left, Main st., Brandon.

53.2 Right at inn; half left, Pittsford, 60.6.

69.1 Right from trolley on Center st.

69.3 Rutland, Center and Wales sts.

LAKE DUNMORE, VT. and LAKE DUNMORE HOTEL

offers tourists going North of Rutland and south of Burlington every comfort, with quiet cool nights, service and a real break in your trip at the most beautiful spot in Vermont, where games, game fishing, bathing, boating, dancing, in short a refined hotel for refined patronage.

Rate $\$ 5.00$ per day and up. American plan

ROSCOE A. MARVEL

Winter connections Qui-Si-Sana-Hotel Spring, Green Cove Springs, Fla.

Rutland, Vt., to Albany, N. Y., 105.5 Miles

0.0 From Wales st., Rutland, west on Center; bear right at station on Grove st.

0.3 Left with trolley on State st., thru West Rutland 4.3; Castleton Corners 14 ; into Fair Haven.

16.4 Bear left at green over bridge; next right.

20.6 Right; left at end 21.3 ; right at x-roads 23.7 .

27.9 Left fork over Hudson river at Whitehall; left Canal st. thru Comstock 35.5; Kingsbury 43.9.

48.7 Keep left of water trough, Hudson Falls; thru Fort Edward 51.3.

51.5 Left with trolley; right over bridge: left at end 58.8 .

62.4 Right fork over river, Northumberland; left at end.

64.7 Straight thru Schuylerville; from trolley.

67.1 Left at end over RR; right 67.8; thru Quaker Springs 71.

73.3 Left fork thru Saratoga Battlefield; join trolley at Bemis Heights 76.5 ; thru Stillwater 79 ; into Mechanicville 82.2.

91.0 Right with trolley on Broad st.; curve left 81.1.

92.7 Right at cross trolley, Ontario st., Cohoes.

92.9 Left, Congress st.; right-left 93.

93.2 Right, Columbia st.; over bridge; left fork 94.7.

95.2 Right fork; left at end 96.4 ; thru Lathams 98.1 .

103.3 Right over viaduct; curve left-right on boulevard.

104.6 Left, Washington st. ; right-left at Capitol on State st.

105.5 Albany, N. Y., State and Pearl sts. 
Boston, Mass., to Concord, N. H., 77.8 Miles

0.0 From Commonwealth ave., Boston, north on Massachusetts over Harvard bridge into Cambridge.

1.4 Right on Prospect; left fork over RR bridge, Somerville.

2.7 Right on Walnut; left on Mystic.

5.3 Right on parkway; right-left at Medford 6.1; right 8.6 ; thru Winchester 9.2 ; right, left at Woburn 11.

12.8 Right fork; thru Wilmington 16.5 ; Tewksbury 22.

25.8 Right, High st.; left on Church across Gorham st., Lowell, 26.5, into Appleton; over RR bridge; right 27.

28.2 Right on Windsor; next left; thru North Chelmsford 31 .

34.3 Right fork, Tyngsboro, into Main st.

41.0 Right fork at monument, Nassau; thru Reed's Ferry 50.2 ; keep right 50.6 .

56.6 Half right on Second st.; cross bridges.

58.0 Right at trolley, Granite st., over bridge.

58.4 Left at end on Elm st., Manchester.

60.0 Right, Webster st., from trolley; left at trolley 60.3.

66.4 Half right thru Suncook 70; Pembroke 72.8 .

74.9 Left; left over Merrimac river bridge.

76.3 Right, Water st. into Main st., Concord, N. H., 77.8.

Concord to Profile House, N. H., 91.0 Miles

0.0 Concord, Capitol; south on Main st.

0.5 Left fork on Fiske; thru Penacook 6.3 and Boscawen 9.2.

18.7 Sharp right downhill at Franklin; thru Franklin Falls 20 .

22.4 Left fork after railroad Tilton; right across bridge 28.5 Winnisquam.

31.5 Left with trolley; keep right of hotel, Laconia 31.6.

31.7 Right with trolley at Tavern into Union ave.; thru Lakeport 33.3.

37.7 Left across bridge into the Weirs keeping left up long hill.

42.2 Left fork after railroad; left at three corners, Meredith 42.6.

50.2 Right at end, Holderness; keep left 50.4 .

54.2 Right at three corners, Ashland; left across Pemigewasset river 55.8 ; then right, crossing railroad at Bridgewater station 56.1 .

59.8 Plymouth, straight on Main st.; thru West Thornton 73 and Woodstock 77.3.

80.9 Left fork at park, North Woodstock, past Flume House 85.9 up long grade through Franconia Notch.

91.0 Profile House at top of grade, White Mountains, N. $\mathrm{H}$.

Profile Ho. to Concord, N. H., 91.0 Miles

0.0 From Profile House, descend long grade thru Franconia Notch, past Flume House 5.1 and thru North - Woodstock 10 and Woodstock 13.5.

31.2 Plymouth, Main st., at High; keep left 31.6.

34.1 Left fork; cross railroad at Bridgewater station 41.

35.3 Left over bridge, Pemigewasset river; right.

37.0 Left fork at hotel, Ashland; left at end 44.8 .

40.9 Left after postoffice Holderness; right at. end 54.4.

48.7 Left at postoffice Meredith; keep left 54.7.

53.6 Right after bridge at The Weirs; follow trolley thru Lakeport 63.5 ; into Union st., Laconia.

59.3 Left at Tavern; right with trolley on Court 69.5.

62.3 Right across railroad Winnisquam; next left; cross railroad at Tilton 68.6 ; thru Franklin Falls 71 .

72.4 Sharp left into Main st., Franklin; thru Boscawen 81.8 and Penacook 84.7; bear left with trolley thru West Concord to

91.0 Concord, Main st., at Capitol. 
Concord, N. H., to Boston, Mass., 77.8 Miles

0.0 From Capitol, Concord, S. ; bear L. into Water st., 10.7.

1.2 Left across Merrimac river bridge; then right thru Pembroke 5.8 and Suncock 8 ; trol. leaves to right 8.9 .

17.2 Keep right leaving trolley on Webster st.

17.5 Left at trolley on Elm st. thru Manchester.

18.6 Right on Granite st. across Merrimac river; left on Second st. 19 into

36.8 Nashua, straight ahead on Main st.

43.0 Left fork, thru Tyngsboro; North Chelmsford 47.

48.0 Left fork; right on Windsor; next left across Gorham st., Lowell; jog L.-R. into Church; R. on High.

52.0 Left on Rogers; left 54.8 ; thru Townsbury 56; Wilmington 61.3 ; right fork 62.2 into Woburn.

66.8 Left at monument thru Winchester 69 ; left 49.8 .

72.1 Right, Main st., Hedford; left on parkway.

72.7 Left at end on Mystic; right at park, cross Broadway.

75.4 Left; over railroad; right fork, Prospect st.

76.6 Left at square, Cambridge, on Massachusetts ave.

77.8 Boston, Mass., at Commonwealth ave.

\section{Boston to Portland Me., 125.2 Miles}

0.0 Fron The Puritan, Boston, east on Commonwealth ave.; left on Massachusetts ave. 0.1 .

1.4 Right at Central sq., Cambridge, on Prospect.

2.3 Half-left over railroad bridge; right on Walnut.

4.5 Right on Parkway; left at end 9.7 past Revere Beach; thru Lynn on Broad st.

15.4 Right at triangle on Nahant st.; left at end.

16.5 Right at monument; right fork Puritan road 17.

18.4 Left on Salem st.; left-right 20.1 .

22.1 Right with trolley, Salem; left at monument 22.2.

22.4 Right at monument around park.

22.5 Left at boulder on Winter; right on Bridge.

23.6 Right at end; half left on Cabot st., Beverly.

46.4 Right beyond monument on Market st., Newburyport; left at end; right across river.

48.8 Right at Salisbury; left at beach 51 .

61.0 The Farragut, Rye Beach, on left.

69.6 Right at cross road over bridge; across bridge with trolley.

71.2 Right around cemetery on South st.

71.5 Left on Junkins; left on Pleasant st. 72.

72.1 Cross Market sq., Portsmouth.

72.6 Cross long bridge, Piscataqua river (15c.).

79.5 Right fork with trolley, York Corner.

80.5 Right at monument, York Village, over railroad.

85.0 Jog right-left at end, York Beach; thru Cape Neddick 86.8; Ogunquit 90.7.

97.6 Left fork under railroad, into Kennebunk.

101.0 Left fork at water trough from trolley.

103.0 Left; thru Biddeford 109.8; Saco 110.8.

124.6 Left on Emery st.; right on Pine 124.8; into Congress to The Lafayette, Congress sq., Portland, 125.2.

\section{Portland to Bangor, Maine, 143.4 Miles}

0.0 From Congress square, Portland, northwest on Forest ave.

0.1 Right, Cumberland ave.; left, Washington st. 1.

2.3 Right with trolley thru Falmouth Foreside 6.2.

12.0 Right fork from trolley, Yarmouth; right fork 12.3 thru Freeport 18; into Pleasant st.

26.5 Right, Main st., Brunswick; left fork 26.7

26.8 Left at Bowdoin College; left fork from trolley 29.2.

35.2 Left at end on Center st., Bath.

35.5 Right at Ferry st. to ferry.

35.8 Left at end over railroad bridge, at once right.

44.8 Right; next left 45.1 ; right fork at green Wiscasset; cross railroad and Sheepscot river. (Cont. on p. 102) 
Portland to Bangor, Me., from page 101

46.9 Left fork beyond North Edgecomb; right fork 48.2.

53.5 Left at end; right at church, Newcastle, 54.3 ; over Damariscotta river into Damariscotta.

54.7 Left fork at bandstand; right at school 54.8 .

55.6 Left fork; thru Nobleboro 59: Waldoboro 65.3.

72.0 Right at end, West Warren; bear left over bridge 75.3; thru Thomaston 77.4 ; into Park st.

81.4 Left at end on Main st., Rockland.

87.8 Right, Central st.; left, Union st., Rockport 87.9

89.3 Left, School st.; next right, Elın st.; bear left on Main st., Camden; keep right at monument.

90.7 Right at end, Northport, down steep grade.

107.9 Right fork on High st., Belfast.

108.7 Right at church, downgrade over bridge.

109.4 Right, Searsport ave.; thru Searsport 114.4.

118.8 Left, Stockton Springs; thru Prospect 123.3.

129.4 Right at end, Frankfort; thru Winterport 130.5; Hampden 137.4.

143.4 Bangor, Main and Union sts.

\section{Bangor to Portland Me., 143.4 Miles}

0.0 From Union st., Bangor, southwest on Main st., thru Hampden 6 ; and Winterport 13.

16.0 Left at postoffice; Frankfort; thru Prospect 20.

24.6 Right at end, Stockton Springs; thru Searsport 29.

34.0 Left over long bridge; bear right at end.

34.7 Left on Main st., Belfast; left fork 38.8 .

42.7 Left after right curve, Northport P. O.

53.7 Left fork at monument on Main st., Camden.

54.0 Left on School st.; next right with trolley.

55.5 Right fork, Center st., Rockport; next left fork across iron bridge, into Main st.

62.0 Rockland; right on Park st., thru Thomaston 66.

67.1 Left fork from trolley; bear right 68.1.

71.4 Left at scales, West Warren; thru Waldoboro 78.1 ; Nobleboro 88.4 .

78.6 Half left at school; thru Damariscotta 88.8; over Damariscotta river into Newcastle.

89.1 Left at church; right 89.9; thru North Edgecomb 96.3 .

98.1 Left fork at green, Wiscassett; right at end.

98.5 Left at four corners; left over railroad; right 107.6.

107.7 Cross ferry to Bath, Me.; out Ferry st.; left at end on Front st.

108.2 Right, Center st., Bath; right fork at monument 108.5.

116.5 Right at trolley; into Main st., Brunswick.

116.9 Left, Pleasant st.; left fork over railroad bridge 119.6, thru Freeport 125.4 ; keeping right 126.7 .

131.4 Left fork, Yarmouth; thru Falmouth Foreside 137.2.

141.1 Left at end, Washington ave.; over railroad.

142.3 Right, Cumberland ave; left Forest ave. 143.3.

143.4 Congress square, Portland, Maine.

\section{Poland Spring to Bangor Me., 117.9 Miles}

0.0 From Poland Spring east thru gates 1.

5.1 Left after underpass at Danville Junction.

10.4 Right at end on Court st., Auburn, over Androscoggin river, into Main st., Lewiston.

11.5 Left fork, still Main; thru Greene 19.2.

19.9 Left from railroad; next right 20.1.

21.1 Left at cross roads; right at cross roads 22.2.

31.1 Right across railroads at Winthrop station; thru East Winthrop 35.5, and Manchester 37.5.

39.6 Right fork, into Western ave., Augusta.

41.8 Half left on Grove st. with trolley.

42.3 Right over Kennebec river bridge; left on Bangor st. 42.6; thru Vassalboro 53.8

Continued on page 105 
Portland, Me., to Boston, Mass., 125.2 Miles

0.0 From The Lafayette, Portland, west on Congress; half left on Pine; bear left on Emery.

0.6 Right on Danforth; cross Fore River 1.4 ; thru Scarboro 5.8, into Main st., Saco.

1.4 Half-right at monument on Elm, thru Biddeford.

24.3 Curve right and keep left of monument, Kennebunk, with trolley; left fork after bridge.

34.5 Right fork at water trough, Ogunquit.

40.2 Right at end, York Beach; at once left thru York Village 44.7, and York Corner 45.7.

51.2 Right fork at Kittery ; cross Piscataqua river.

53.1 Left at Market sq., Portsmouth, on Pleasant st.

53.3 Right on Junkins; right at end 53.7.

54.0 Left around cemetery on Miller (trolley).

74.2 Right at end, Salisbury Beach, with trolley.

76.4 Left at pump, Salisbury; keep left of monument; cross river into Newburyport.

78.5 Left with trolley on Merrimack st., two blocks.

78.6 Right on Market; left at end on High 78.8.

99.0 Left with trolley into Cabot st., Beverly.

102.6 Left on Winter st.; right at boulder 102.8 .

102.9 Left at monument; right at trolley 103.3.

103.3 Left on Central ave., Salem, with trolley.

104.3 Right fork at Normal School; left-right 104.

107.8 Left fork at monument; keep left 108.8.

109.8 Right on Nahant st.; left at small park Lynn 110.3; keep left along Revere Beach 115.

115.5 Right at circle on parkway; left at end 120.7 .

122.6 Left at end on Bow st.; right after railroad bridge.

124.2 Left on Massachusetts ave., Cambridge.

125.2 Commonwealth ave., Boston, Mass.

\section{Tbe Copley IDlaza}

New England's Finest Hotel

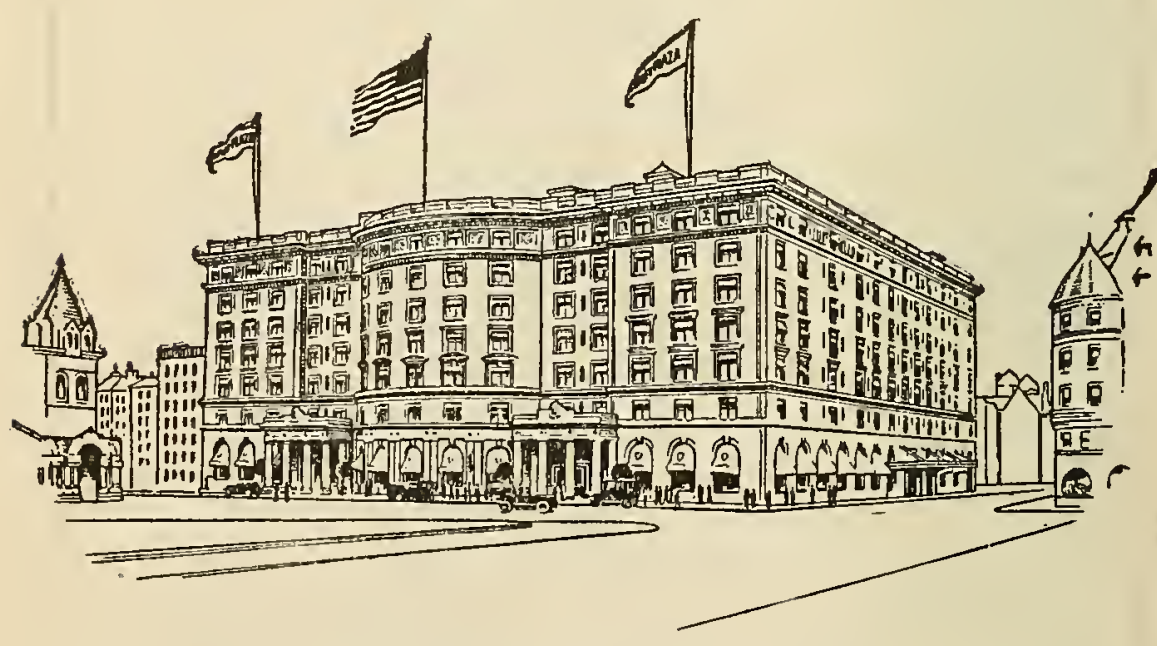

COPLEY SQUARE

Starting Point and Terminus of all Touring Roads to and from the city

Motorists will find here the finest Parking Space in Boston 


\title{
Poland Spring to Bangor, Me., 117 Miles
}

\author{
Continued from page 102
}

60.2 Left at Winslow; cross bridge and railroad; left on Clinton ave. 60.9.

61.1 Right at end on Main st., Waterville; half right on Elm 61.5.

64.6 Right across bridge at Fairfield; left at P. O. Benton 65.1 .

65.2 Next right at trolley; left at end, Benton.

67.9 Right fork thru Clinton 71.2; Burnham 77.1.

84.6 Right on Grove st., Pittsfield; right 91.3 ; thru Newport 91.8 , and Etna 100.1 .

101.2 Left; left at Carmel 103.5; left 103.7.

110.6 Right fork, Herman, direct into Hammond st.

117.9 Bangor, Hammond, State and Main sts.

\section{Bangor to Poland Spring, Me., 117.9 Miles}

0.0 From State st., Bangor, west on Hammond st.

7.2 Left fork at monument, Herman; right fork 13.6.

14.0 Right at end; right at Carmel 14.4; half right 15.1 ; thru Etna 18.

26.1 Left at Newport; left 26.8; follow wires.

33.3 Left at end; thru Pittsfield; Clinton 46.7.

51.1 Right at trolley beyond Benton; left 52.7.

53.3 Left at trolley, Waterville, into Main st.

53.8 Right around postoffice, West Benton into Fairfield.

56.8 Left on Bridge st., over Kennebec river.

57.2 Right with trolley; right at end, Winslow 57.7, thru Vassalboro 64.

75.3 Right, Coney st., over bridge Kennebec river.

75.6 Left, Water st.; curve right into Grove st.

76.1 Half right on Western ave., Augusta, with trolley; thru Manchester 80.4 .

86.7 Left after railroad, Winthrop; right fork 92.1.

95.7 Left at four corners; right at four corners 96.8 .

97.8 Left at end; right along railroad 98 ; right fork at Greene 98.7.

106.6 Thru Lewiston on Main st. over bridge into Auburn.

107.5 Left after railroad, Washington st.; left fork 108.

112.8 Right under railroad at Danville Junction.

117.8 Poland Spring, end of road at hotel.

\section{TROUBLE RULES FOR TROUBLESOME AUTOMOBILES}

I. Gasoline-Is tank full? Are pipes clean? Is gasoline clean? Is carburetor clean? If carburetor needs adjusting do it, otherwise leave it alone. Does manifold leak?

2. Compression-To test: Open all petcocks except the one on cylinder to be tested; crank engine, noticing how strong the compression is in each cylinder.

3. Ignition-A. Test for a spark by taking wire off any plug; hold wire about $1 / 8$ of an inch from plug; crank engine with switch on. Spark should jump to plug.

B. Are the batteries run down? Does the vibrator (if any) buzz? Is the timer clean? Do timer points make good contact?

C. Are any wires loose, burned, wet, broken or short circuited? Are spark plugs clean and are points I-50 of an inch apart?

D. Does magneto armature revolve? Is safety spark gap clean? Are interrupter points clean and adjusted right? Do all brushes make good contact? Is distributer clean? Is distributer motor loose, broken, or making poor contact?

Loose wires and terminals and neglected batteries cause much trouble.

Put distilled water in your batteries every two to four weeks. 
Pittsfield, Mass., to Rutland, Vt., 96.6 Miles

0.0 From park, Pittsfield, north on North st.

0.8 Right fork at hospital; next right, Tyler st.

3.3 Left at six corners; left from trolley 5.1.

15.3 Left on Columbia st., Adams; right fork 15.7.

20.2 Left fork, Ashland st., with trolley.

21.5 Left on Main st., North Adams; left fork 22.8.

27.0 Right on North st., Williamstown, around hotel; bear left at end; keep right fork 27.9 .

31.8 Right at Pownal; thru Pownal Center 34.4.

39.9 Thru Bennington on North st.; right 40.1 ; thru South Shaftsbury and Shaftsbury Center 48.6.

49.3 Right fork, across railroad at Shaftsbury; thru Arlington 55 .

63.9 Manchester, right fork at library; curve left-right at Manchester Center 65.4; thru East Dorset 70.2.

76.8 Right over bridge at Danby; left fork 79.2.

87.1 Left at far end of Wallingford; cross RR; then right at end.

95.3 Right thru covered bridge; left at end 96.1.

96.3 Right fork, Wales, to Center st., Rutland 96.6.

\section{The Richmond Hotel}

\section{Home of the Mohawk Trail}

Rooms Single and En Suite, with bath
Dining Service Table d'Hote and a la Carte

NORTH ADAMS, MASS.

George R. Bush $-\quad-\quad-$

Manager

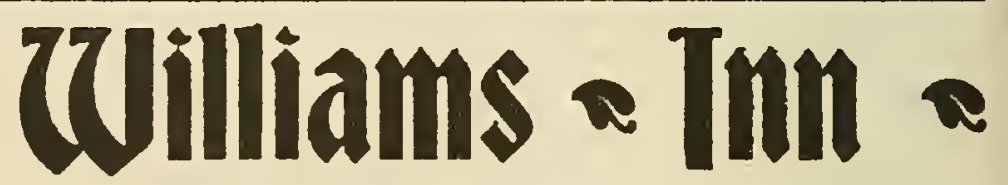

Beautifully nestling in the alluring Berkshire Hills

Noted for Simplicity and Homelike Environment

We Please Particular People

WILLIAMSTOWN, MASS.

L. G. TREADWAY, Manago

AFFILIATED WITH

Hshfield Бouse, Hshfield, mass. Dorset Tnn, Dorset, Ut.

WILLIAMS INN GARAGE

GEO. W. GRUNDY, Proprietor

WILLIAMSTOWN, MASS.

Corner Main and Water Streets

Full Line of Supplies Garage Open All Night

CASTOR OIL ON EYELASHES

Every motor car rider has experienced the discomfort of dust in the eyes, and also from the effects of cold winds. A suggestion has been made that these discomforts may be greatly alleviated by applying castor oil along the eyelashes. This, it is claimed, will catch most of the dust before it can enter the eye, and also it protects the eyes from the chilling effects of the wind. 
Rutland, Vt., to Pittsfield, Mass., 96.6 Miles

0.0 From Center st., Rutland, south on Wales st.; bear left at end; next right over RR bridge, Madison st.

1.0 Left after covered bridge on Kelley road.

10.5 Left across railroad; right, Main st.. Wallingford.

20.8 Left after bridge, Danby; thru East Dorset 28.

31.5 Left at end; right of park, Manchester Center.

34.7 Manchester; right fork leaving town.

31.0 Left fork; thru Arlington 42.6; Shaftsbury Cen. 49.

52.2 Left fork; thru South Shaftsbury; under RR 55.7.

55.8 Left at trolley; right fork from trolley 56.1 .

57.0 Curve left on North st.; thru Bennington 57.7; Pownal Center 63.2, and Pownal 65.8.

68.9 Right fork; keep right into North st.

69.6 Left on Main st., Williamstown, Mass.

75.1 Right, Ashland st.. North Adams, with trolley.

77.5 Right across trolley; left 78.3.

78.4 Right with trolley, Commercial st., Adams.

86.2 Left fork from trolley; left fork, Berkshire 90.2.

93.3 Right at six corners. Dalton st.; left at end 95.7.

96.6 Pittsfield, Mass.; park on left.

\title{
The
}

PITTSFIELD, MASS.

\author{
AMERICAN PLAN
}

34th Season. Open May 26th to November 1st

Booklets of Road Maps on application

ARTHUR W. PLUMB

\section{The Garage of Springfield, Mass.}

KIMBALL GARAGE

One block from Kimball Hotel

FIRE PROOF

STODDARD MOTOR CAR CO., Springfield, Mass.

\section{Putnam Auto Salvage \& Repair Corp.}

178 Clove Ave., HAVERSTRAW, N. Y.

Tel: Haverstraw $139 \mathrm{~L} 4$

New York Office, 302 Broadway. Tel. Worth 0444

\section{AUTOMOBILE SPECIALISTS}

Repairing and Overhauling. All work Guaranteed.

Estimates cheerfully furnished.

WE BUY WREGKED CARS 
Springfield, Mass., to Keene, N. H., 75.6 M.

0.0 From Springfield, north on Main st.; left fork over bridge Connecticut river; right, Riverdale ave., thru outskirts Holyoke 8.4.

16.2 Right over bridge; left on Pleasant across Main st., Northampton 16.8; left fork 19.4; right fork 22.1, South Deerfield with trolley across covered bridge, then left.

37.1 Thru Greenfield on Federal st.

43.4 Right at hotel, Bernardston; right 9.6.

47.4 Right; cross Connecticut river 47.8; under RR 48.4.

49.5 Left thru Northfield, and East Northfield 51.6.

56.3 Right at end thru Hinsdale, along river.

62.3 Right at end across bridge; next left at monument, Winchester; thru West Swansea 70.

75.2 Left at end, Washington st. to monument, Keene 75.6.

\section{Thotel Thonotuck}

P. J. BEHAN

HOLYOKE, MASS.

Fireproof

Roof Garden

European

A Most Desirable Stopping Place

for Business Men and Tourists

\section{Woerg's Garage}

Around the Corner from the Nonotuck

Keene, N. H., to Springfield, Mass., 75.6 M.

0.0 From monument, Keene, south. on Washington st.

0.4 Right beyond church on Winchester st.

13.2 Right at monument, Winchester; next left.

19.3 Left beyond P. O. Hinsdale; thru Northfield 26.5.

27.0 Right under railroad and across Connecticut river.

28.2 Left at three corners; left around School 32.

32.2 Left at end, Bernardston, into Federal st.

38.3 Cross Main st., Greenfield; right across bridge.

43.7 Left at end of Deerfield; follow railroad thru South

Deerfield 47.6 into King st., Northampton, across Main st. 58.8 .

59.4 Right across small bridge; next left thru Holyoke 67.2 .

75.1 Left across Connecticut river into Springfield 75.6.

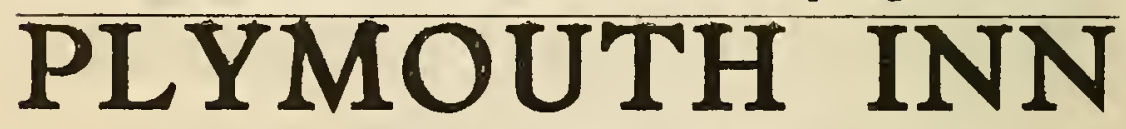

West and Greene Sts.

Northampton, Mass

Modern and appealing. Away from the noise of the business section. American or European plan.

\section{WILLIARD A. SENNA}

Dríme Jorest Inn

Summerville, South Carolina

Same Management 


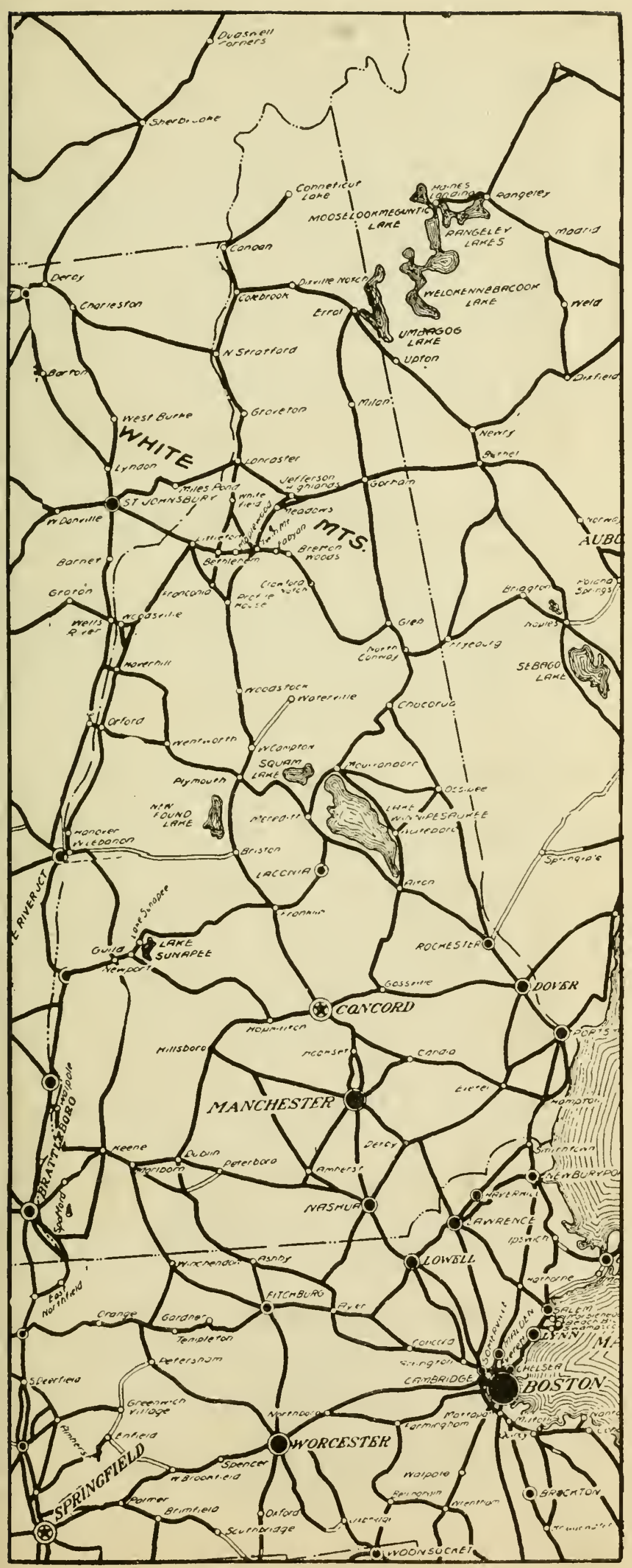




\section{Keene to White Mountains, N. H. 143.7 M.}

0.0 From monument, Keene, north on Washington st.

8.6 Right at Gilsum; left fork 8.9.

15.7 Left at four corners, Marlow; right at inn; next right fork; right fork 29.6.

34.8 Thru Newport; right fork 36.5 ; left fork 38.3 .

38.4 Right, Croydon Flats; right at end, Croydon.

45.3 Left fork at postoffice Grantham; left fork 58.5.

59.4 Right-left at Lebanon; thru West Lebanon 63.

66.9 Right at Hanover green; next left, College st.; thru Lyme 77.6 , and Orford 85.

91.1 Right fork, Piermont; thru Haverhill 95.6.

97.7 Right fork; into outskirts Woodville.

103.7 Right with blue poles; left fork 107.5.

108.9 Right fork at Bath; thru Lisbon 114.5.

116.6 Left fork over railroad; right 123.7.

125.2 Right at monument, Littleton; left 125.7.

130.2 Thru Bethlehem, and Maplewood 131.6.

133.3 Right at Bethlehem Junction; left fork 138.2.

138.5 Pass Twin Mountain Hotel to Bretton Woods station 143.7.

\section{GRANLIDEN HOTEL}

LAKE SUNAPEE, N. H.

One of the handsomest hotels in the country. Accomodates 300 guests. Delightful place to stop on your way to or from the White Mountains or for the season. Good Golf Course. Saddle Horses. Fine Tennis Courts. Boating, Bathing, Fishing. Dancing each evening. Cottages for rent by season.

For illustraled Booklet and Terms apply

J. THOMAS RUSSELL, Prop.

Hotel Weylin, 54th St. at Madison Ave., New York City also Hotel Russell, Saratoga Springs, N. Y.

\section{Bretton Woods to Keene, N. H., 143.7 Miles}

0.0 From Bretton Woods station, north along railroad, past Twin Mountain House 5.2; right fork 5.5 .

10.5 Left at Bethlehem Junction; thru Bethlehem 13.5.

18.5 Left at monument, Littleton; left fork 19.0.

19.9 Left at end along river; thru Lisbon 29.2.

30.3 Left under railroad; left fork at Bath 35.1.

40.0 Left at end from Woodsville; left fork 42.

47.5 Right fork; thru Haverhill 48.1.

52.7 Left fork at Piermont P. O.; thru Orford 58.7.

66.1 Half right at hotel, Lyme; next left fork.

76.7 Right beyond green on Wheelock st., Hanover.

76.8 Left on Main st.; thru West Lebanon 80.6.

84.3 Right-left at park, Lebanon; right fork 86.

86.6 Right along brook; thru Grantham 98.4.

102.0 Left at Croydon; left at Croydon Flats 105.3.

108.9 Thru Newport; right fork 114.4.

128.2 Left at inn, Marlow; right after bridge.

135.1 Left at end, Gilsum to monument, Keene, 143.7.

\section{LAKE DUNMORE, VT. and \\ LAKE DUNMORE HOTEL}

On a wonderful route. Same distance Rutland to Burlington, Vt. 
Niagara Falls, N. Y., to Toronto, Can., 82.9

0.0 Niagara Falls, International Bridge.

1.0 Turn L from River Road at forks. (Church.)

1.3 End of street, turn $\mathbf{L}$. Station at $\mathbf{R}$, crossing R.R. (1.9), straight end of road (2.5). Turn $\mathbf{R}$, then $\mathbf{L}$ (3.0), crossing R.R. (3.3).

3.6 Turn $\mathbf{R}$ to pavement.

5.0 Turn L. 6.0 St. Davids, 6.7 Sharp L.

11.1 Curve $\mathrm{L}$ by church, at 11.4 curve $\mathrm{L}$ over New Welland Canal. 12.9 St. Catharines.

14.2 Trolley station on L. Straight ahead. High Level Bridge at 15.2. 18.0 Caution-bad hills. 21.3 Short turn, bad hill. 22.8 Jordan. 26.7 Beamsville.

28.7 Cross radial, keep to $L$, road to $R$ (30.2) going to Grimsby Beach Park. 31.5 Grimsby.

32.1 Sharp turn to $\mathbf{L}$ over radial, curving $\mathbf{R}$ at 32.2.

36.7 Winona. Road to $\mathbf{R}$ to Toronto, via Barton St. and Burlington Beach. Straight thru cross-roads at 37.0 , $38.1,38.2$, to 39.4 Fruitland.

42.0 Stoney Creek. Turn sharp $R$ (42.3). (Ch.)

43.0 Bad hill. Curve $R$ (43.2).

43.6 Barton St. Straight on thru viaduct (43.9). Turning sharp L (44.4)-Lake ahead.

45.8 Road to $\mathrm{L}$ to Hamilton.

45.9 Burlington Beach town line.

50.3 Dangerous crossing. Turn $\mathrm{R}$ on to Toronto-Hamilton Highway. 56.8 Bronte.

59.9 Cross bridge over Oakville Creek. 60.8 Oakville.

64.7 Curve $L$ then $R(64.8)$.

68.6 Lorne Park. Store on corner.

69.2 Cross bridge over Credit River at Port Credit.

73.3 Long Branch on R. 74.7 Thru New Toronto.

79.3 Cross viaduct at Sunnyside Station; $R$ on King st. 82.9 King and Yonge sts., Toronto.

\section{'The Prince George Hotel}

Corner King and York Streets

Toronto, Canada

Quiet Homelike Refined

250 rooms. 100 with private baths

European Plan $\$ 2.50$ per day and upwards

SAMUEL H. THOMPSON, Proprietor

\section{Uhe Quern's $\underline{\underline{\text {Taronto, Ont. }}}$}

Celebrated for its home comforts, perfect quiet, good attendance and the peculiar excellence of its cuisine. Delightfully situated near the Bay on Front Street. Convenient to business center, railroad depots. Steamboats, etc. 
Toronto, Can., to Niagara Falls, N. Y., 82.9

0.0 Toronto, King and Yonge st., W. on King st.

3.6 Sunnyside Station. Left over iron bridge, follow Lake Shore road to Humbler River.

6.0 Cross Mimico Creek 8.2 thru New Toronto.

10.3 Cross bridge over Etobicoke Creek.

13.7 Cross Credit River at Port Credit.

15.3 Lorne Park. Store on corner.

22.1 Oakville (Village Inn to left).

23.0 Cross Oakville Creek. 26.6 Bronte town line.

32.6 Burlington town line.

34.0 Turn L over dangerous R.R. crossing to macadam road, Brant House (34.1) on R cross R.R.'s at 34.5.

38.0 R.R. crossing. Road to $\mathbf{R}$ goes to Hamilton.

39.9 Sharp right, under R.R. viaduct at $(40.4)$.

40.7 At four corners turn L. Stone road at

46.7 Winona. 'Cross R.R.'s at 48.4, 48.8 and 50.1.

50.7 Curve $L$, then $R(50.8)$ over radial and thru village of Grimsby. Bad hill (54.2) and R.R.'s at 54.3 and 54.9.

55.2 Beamsville. 601 Jordan, curve and hill 61.5.

67.3 St. Catharines. Cross bridge over R.R.

67.9 Turn $L$ pavement one st. for High level Bridge. St. Paul str. past radial station, follow trolley tracks.

69.3 Sharp $R$ down hill, under viaduct (71.0) continue with trolley to (71.3) Merriton, Main st.

71.7 R.R. crossing, end of pavement. Follow trolley, curving $\mathbf{R}$ along Welland Canal.

72.8 Under viaduct into Therold.

73.0 Turn $\mathrm{L}$ trolley station on $\mathbf{R}$, next str., sharp $\mathbf{R}$.

74.1 Cross R.R. leaving trolley. Turn $L$ crossing two bridges and R.R.

76.1 Turn L crossing R.R.

79.9 Lundy's Lane. 81.6 Niagara Falls south.

82.5 Victoria Park. Turn $R$ over R.R. passing Cliffton Inn. Turn $L$ on River Boulevard to

82.9 International Bridge-Niagara Falls.

\section{Motor Headquarters for the Niagara Peninsula

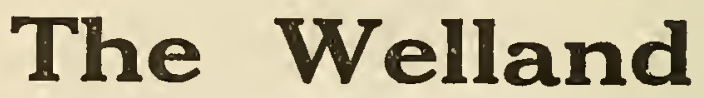

St. Catherines

Advise ahead for meals and rooms

12 miles from Niagara Falls Famous Mineral Springs

American Plan $\$ 4.00$ up

\section{THE INN}

Rate $\$ 1.50$ per day and up

Location, finest residential section in the city

Hot and cold water in every room

Make this hotel your headquarters

H. J. ABBO'TT

NIAGARA FALLS, N. Y.

\section{THE PROSPECT HOUSE}

NIAGARA FALLS, N. Y.

Cor. Jefferson Ave. and Second St. One block from N. Y. Central, two blocks from the Falls.

Strictly first class. Every other room with bath. Open all the year.

Rates, \$2.50 up. European. H. W. ISAACS, Proprietor

After viewing the Falls visit a most instructive and interesting feature

NIAGARA FALLS MUSEUM

Price Twenty-five Gents 


\section{TROUBLES AND THEIR SOURCES}

A Great Deal of time is wasted by many motorists when car develops trouble, by not giving sufficient thought, if any, to the symptoms shown from the time trouble was first noticed to the time an attempt is made to set matters in order. Diagnosing automobile troubles requires thought and reason.

Never Tamper with or destroy the arrangement of parts of the engine or car unless fully satisfied of the real trouble. Note the symptoms and endeavor to discover the seat of the disturbance. If not successful in this see the nearest dealer or some one qualified by experience and have, whatever needs attention adjusted in a correct manner.

Make It a Point to understand the car thoroughly. Remember when diagnosing trouble there are two essentials necessary-first, gasoline; second, a spark.

Gasoline, in vapor form, must reach the inside of the cylinders and the spark must be there at the proper time to ignite the gas. If you have both, something is bound to happen, even though it is but a single explosion. Bear in mind that even though there be gasoline and spark, the engine will not run properly unless the cylinders receive the gas mixed in the correct proportion with air.

Remember that if an engine is not properly lubricated and cooled, it will heat.

In Summing $U p$ the chief troubles it is found that most all are due to Ignition, Carburetion and Lubrication.

If trouble occurs, find out which is responsible.

Listed Below is a summary of troubles and their causes.

Engine Fails to Start: Gasoline exhausted; mixture too lean; water in gasoline; supply shut off; water in carburetor; carburetor frozen (winter); switch off; dry cells or battery weak or not properly connected.

Engine Runs Irregularly (Missing): Usually ignition, caused by (a) dirty spark plugs, (b) improper gap in spark plugs, (c) broken porcelain in spark plugs; poor compression; leaky intake manifold; bad mixture; valves set too high or too low; valve sticking.

Engine Stops Suddenly: Usually ignition; water or dirt in gasoline; overheated-lack of oil or water; gasoline tank empty (usually causes engine to stop gradually).

Overheating: Lack of oil or water; loose fan belt; carbon; spark retarded; mixture too rich; sediment in radiator.

Engine Knocks: Carbon; overheating; loose connecting rod or wrist pin; broken piston ring; spark too far advancerl.

Engine Hisses While Running: Spark plug porcelain broken; exhaust pipe cracked or joint loose; spark plug loose in cylinder.

Excessive Smoke from Muffler: Over-lubrication (blue smoke); mixture too rich (black smoke).

Lack of Power: Loss of compression; carburetor flooding; weak ignition; lack of oil or water; brakes dragging; flat tires; lack of gasoline (if line is stopped motor will spit back through carburetor with throttle open).

Back Firing (Through Carburetor): Intake valve or exhaust valve holding open.

Engine Spits Back Through Carburetor: (Erroneously called "backfiring.") This usually indicates too weak a mixture and may be caused by-Carburetor adjusted for tou much air and too little gas; dirt in gasoline passage or nozzle; air leak around intake to carburetor.

Gasoline Record: The Average Motorist confines himself to one brand of gasoline believing that he obtains maximum efficiency.

A Simple Method of gasoline accounting that shows actual results.

Do This: Fill Motor Tank Full First. Fill tank Full whenever a pumping is made. Always Make Record at tume of refilling. 


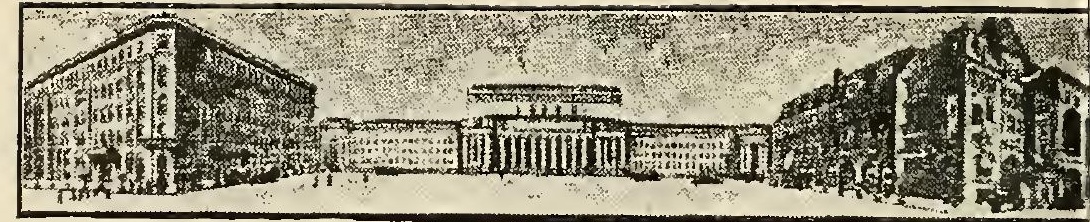

To the Subscribers of

New York--New England--Canadian Motor Tours: THOSE travelling by automobile, whether for business pleasure, often find it difficult to keep within the limit ordinary expenses while touring.

We, as hotel keepers, suggest that you patronize

\section{The Walker House or The Hotel Carls-Rite}

where we specialize on the American Plan. Few Hotels on t American Continent give the same value in Dining Room Servi and Comfort, for the price you pay, as

THE WALKER HOUSE or HOTEL CARLS-RITE

\section{"Toronto's Two Famous Hotels"}

Our Table is the foundation of our enormous business. pay particular attention to Ladies and Children travelling alon EUROPEAN PLAN IF DESIRED

GEO. WRIGHT \& E. M. GARROLL, Proprietors

Toronto to London, Ont., 122.0 Miles

0.0 From Yorge st., Toronto, west on King st.; thr Mimico 6; New Toronto 8; Port Credit 13; Oal ville 22 ; Burlington 33.

40.1 Left over bridge, on York st., Hamilton.

42.9 Right at end on James st.; next right, King st.

46.5 Left across RR; thru Alberton 55; Cainsville 63 Brantford 66; Burford 75; Eastwood 88; to Wooc stock 92.4 .

92.9 Half left, Ingérsoll rd.; thru W. Oxford 97.

93.7 Right on Thames st., Ingersoll; left at church 10

107.8 Left over bridge; Thames ford; thru Crumlin 11

121.8 Left on Richmond st. to York st., London 122.

\section{London to Toronto, Ont., 122. Miles}

0.0 From York st., London, north on Richmond.

0.2 Right on Dundas st.; thru Crumlin 6.

14.2 Right after bridge, Thamesford, into Ingersoll.

20.0 Right at end, Thames st.; left, Charles st. 20.3; thr West Oxford 25.

29.1 Half right at end thru Woodstock; thru Eastwoo 34; Burford 47; Brantford 56; Cainsville 59; Al berton 67 .

75.5 Right at end; right over RR on King st. 77.8.

79.0 Left on James st., Hamilton; next left on York.

81.8 Right fork, thru Aldershot 84 .

85.7 Half right thru Burlington 89; Oakville 100 Port Credit 109; New Toronto 114, and Mimic 116 ; into King st.

122.0 King st., Toronto, at Yorge st.

\section{Toronto to Kingston, Ont., 162. Miles}

0.0 From York st., Toronto, east on King st.

3.7 Half left; thru Scarboro 12; Dunbarton 19.5 Whitby 28.5 ; Oshawa 32 ; Bowmanville 42 ; New castle 47.

60.0 Right at Welcome; left 62.4; right at end, Por Hope 63.3.

63.6 Half left under bridge; right 68.7 ; bear left, thri Cobourg 70.

78.9 Half right at cemetery; left at Colborne 85.5.

93.7 Right at end, next L. at Brighton; thru Trenton 103 Continued on page 116. 


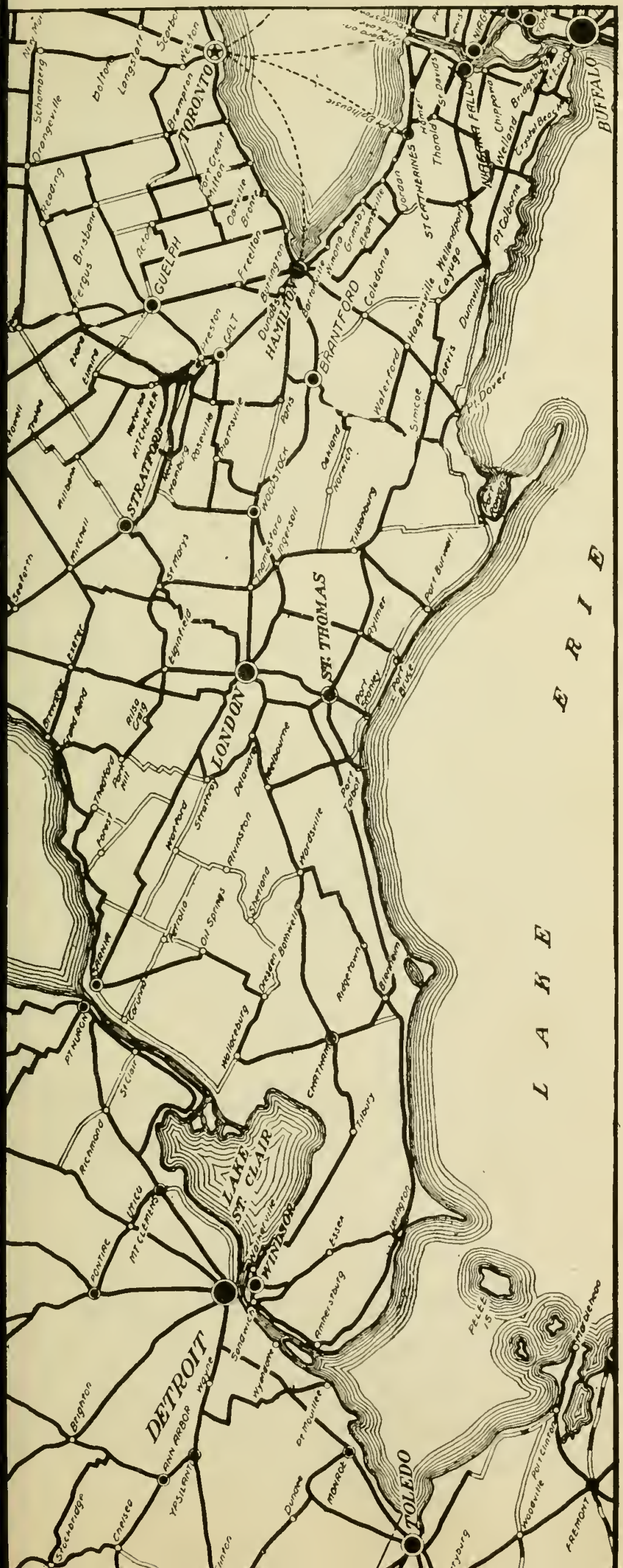




\section{Toronto to Kingston}

Continued from page 114.

103.5 Right at end; right, Front st., Belleville 114.

103.7 Half left, Dundas st.; left; Shannonville 122.6; thru Marysville 129.

134.5 Left; bear left at church 131, into Napanee.

137.6 Half left under RR; next right; thru Odessa 150.

158.5 Half right at Cataraqui into Princess st.

162.0 Left on Wellington st., Kingston, at City Hall.

\section{Hotel Arlington}

King St., West-Corner John Street

TORONTO

Main approach to city. Only five blocks to business center and leading theatres. Under new management. Modernized-Newly furnished

European Plan

\section{Kingston to Toronto, Ont., 162.0 Miles}

0.0 From City Hall, Kingston, west on Princess st.

3.5 Left at Cataraqui P. O.; thru Odessa 12.

24.3 Left at end under RR; next right, Napanee.

24.9 Half right at church; right at end, thru Marysville 33.

39.4 Right at Shannonville, coming into Dundas st.

47.8 Half right on Front, left on Bridge st., Belleville 48.

58.5 Left, cross long bridge at Trenton 59.

68.3 Right; next left at Brighton.

76.5 Right at park, Colborne; thru Cobourg 92.

93.2 Left at cross-road; left at Port Hope 98.7.

99.6 Right; left at Welcome 102; thru Newcastle 115; Bowmanville 120 ; Oshawa 130 ; Whitby 133.5 .

140.2 Half left; thru Dunbarton 142.5 and Scarboro 150.

158.3 Half right at end on Queen st.; left fork after bridge on King st.

162.0 Toronto, King st., at Yorge.

\section{VICTORIA GARAGE}

\section{Stanley Street - Montreal}

Rear Windsor Hotel-Three blocks from Queen-Two blocks from Ritz-Carlton

Storage 300 cars, on the level

\section{KEEP MUFFLER CUT-OUTS CLOSED}

Horns are intended for protection and not for amusement. Use them with intelligence.

Do not turn from a parked position into the traveled highway without first looking to the rear for following traffic.

Speed means serious and even fatal accidents, increased tire and repair expense, nervous prostration and loss of money, time and pleasant disposition. 


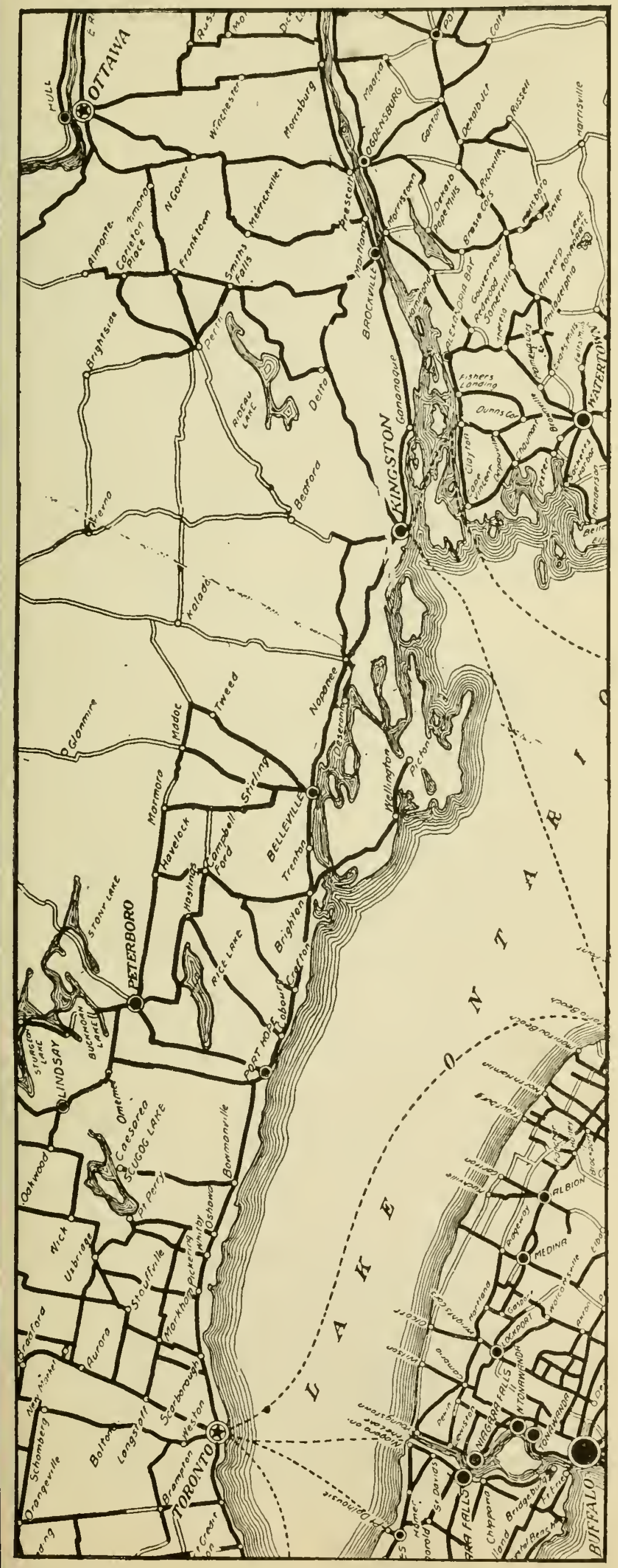




\section{Montreal to Kingston, Ont., 195.3 Miles}

0.0 From Dominion sq., Montreal, west on Dorchester.

1.3 Left on Green st.; right on St. James 1.6; thru Lachine 8 .

23.5 Left to ferry; left at hotel Isle Pierrot.

24.6 Right; right 27 to ferry; straight on.

33.6 Right at Cascades Point hotel along canal.

63.9 Left at cross street, Lancaster, Ont.

64.8 Right at S. Lancaster P. O. thru Summerton 70.

80.7 Right on Pitt, left on Second st., Cornwall, 81.

86.5 Left; thru Aultsville 99; Cardinal 121.

130.9 Prescott; straight on across George st. thru Maitland 138 (left leads to ferry for Ogdensburg, N. Y.)

143.1 Straight thru Brockville; half right 145.

149.2 Half left at Lyn; thru edge of Mallorytown 158.

165.4 Half right; left at Lansdowne 167.

176.9 Right after RR and bridge, Gananoque.

184.3 Half left with wires; left past College 193.7; cross bridge Cataraqui river; left-right at end.

195.1 Left on Wellington st., Kingston, to City Hall 195.3.

\section{REVERE HOUSE}

On the St. Lawrence BROGKVILLE, ONT.

Rates $\$ 4.00$ up American Plan

Rooms with Running Water and Bath ROBERT JOHNSTON

\section{Grand Central Hotel}

Home of Tourist and Traveler

Rates $\$ 3.00$ up

MCAULEY \& POWERS
American Plan Brockville, Ont.

Kingston, Ont., to Montreal, P. Q.,195.3 M.

0.0 From City Hall, Kingston, north on Wellington.

0.2 Right; on Place d'Armes; over long bridge.

1.6 Right with wires; half-right at end 9.4.

18.4 Left on King st., Gananoque over bridge.

27.4 Half-left; right at Lansdowne 28.3; thru Mallorytown 37.

46.1 Right fork at Lyn; thru King st., Brockville 52.

64.4 Cross George st.. Prescott; thru Cardinal 74.

114.3 Right, Pitt st., Cornwall; left at end on Water st.; thru Summerton 125.

130.5 Left at S. Lancaster; right at store, Lancaster 131.4.

161.7 Left at end to ferry 164.3; right on landing.

171.6 Second ferry; straight on thru Lachine 187 into St. James st.

193.7 Left, Green st.; right Dorchester 194, to Dominion sq. 195.3.

\section{The British American Hotel KINGSTON, ONT.}

Recently refurnished under new management

The COMMERCIAL man and TOURIST may feel assured of receiving the best accommodation Kingston affords.

H. G. GOCHRANE Prop. and Mgr.

Rouses Point, N. Y., first stop in the U. S. Customs Office. Next stop, WINDSOR HOTEL. 


\section{Quebec to Montreal, P. Q., 180.5 Miles}

0.0 From Chateau Frontenac, Quebec on left S. O. St. Louis Stand Grande Alle.

Left, thru St. Foye 2.1.

5.6 Right; left at Lorette 8.3 ; thru St. Augustin 14.5 and Les Ecureuils 27.5.

37.6 Left at end; Portneuf; thru Deschambault 41.5.

57.2 Left at Ste. de la Parade, over bridges.

61.7 Left to ferry (25c.) ; straight on thru Champlain and Cap de la Madeleine 70.5.

81.5 Left at trolley over bridges on St. Maurice st.

82.8 Left on Laviolette ave.; Three Rivers.

83.4 Right on Notre Dame st.; keep left at church 92.5 .

01.0 Right at monument beyond Yamachiche.

07.2 Right over bridge at Louiseville.

11.6 Right at end, Maskinonge; thru St. Barthelmi 118.

23.2 Left at end; right over covered bridge 125.2.

28.8 Left at Berthier; thru Lanoraie 138.5.

51.4 Right; left over bridge 154.1 on boulevard.

55.0 Left at L'Assumption on Notre Dame; right at end.

63.5 Right fork Charlemagne, under RR, next left, with RR over bridges.

64.9 Right at end; left at end; right and S. O. Notre Dame St. arriving to right at Fork by old City Prison into Craig (later becoming St. Antonie St. to Windsor St., 180.5 .

Left for Burlington or Plattsburg; left for Queens, 1 block; right for Windsor, 2.. blocks, and beyond for Ritz-Carlton.

\section{anada Hotel} OPPOSITE C. P. R. STATION

15 ROOMS WITH PRIVATE BATH

45 ROOMS WITH RUNNING WATER

Headquarters for Rotarians

European Plan

Night and Day Service

Garage close to Hotel

C. H. MOINEAU - - Proprietor

Les Trois-Rivieres, Quebec On the Montreal-Quebec Highway

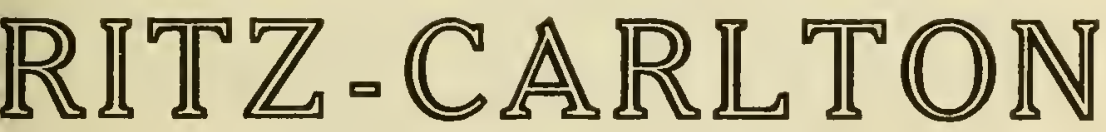

Montreal's Exclusive Hotel

Delightfully located in the residential section, away from the noise and grime of the business section, yet easily accessible to theatres, shops, etc. . .

Excellent Garage Accommodations Unsurpassed Cuisine and Service 


\section{Montreal to Quebec, P. Q., 180.5 Miles}

0.0 Montreal, from Windsor and St. Antonie Streets straight on east along St. Antonie, becoming Craig, then Notre Dame Streets.

15.6 Right under $R R$, left with $R R$ over bridges.

16.8 Right at end, Charlemagne; thru St. Paul 18.5.

25.4 Left, Notre Dame st., L'Assumption.

25.5 Right on boulevard; right at end of bridge 26.4 .

29.1 Left at end; thru Lavaltrie 36; Lanoraie 42.

51.7 Right into Berthier; left over covered bridge 52.

54.1 Left at end; right 55.3 ; thru St. Guthbert 56.

57.3 Right; thrn St. Barthelmi 62.4.

68.9 Left at Maskinonge; left at end, Louiseville 73.3.

79.5 Left at mon., Yamachiche; keep right of church 88 .

97.1 Left on Laviolette st., Three Rivers.

97.7 Right, St. Maurice st., over bridges.

99.0 Right; thru Cap de la Madeleine 101 to ferry 118.4 (25c.)

118.8 Right at end; right at end, Ste. Anne de la Parade 123.3; thru Deschambault 139.

142.9 Right at Portneuf, over bridge; thru Les Ecureuils 153 , and St. Augustin 166.

172.1 Right at end, Lorette; left at end, St. Foye 174. Right at Monument and thru Battlefields Park and along Grande Alle to Chateau Frontenac.

\section{Cbateau}

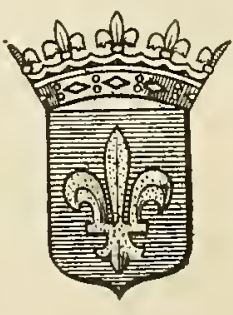

\section{Leading Hotel in Three Rivers}

Garage in Connection

Meals Served from 6 a. m. until 10 p. m.

Unexcelled Accommodation for Automobile Parties

Toledo to Cleveland, Ohio, 124.1 Miles (via Sandusky)

0.0 Jefferson ave., Toledo, north on St. Clair.

0.4 Right on Cherry st., over Maumee river.

1.4 Left, Starr ave.; next right, E. Broadway.

7.9 Right across railroad-left; next left 8.4 thru Latchie 11.4 .

13.4 Right at four corners; left at next four corners 14.3.

17.2 Right at six corners; thru Genoa 18 ; trolley leaves 20.

22.7 Left at trolley; right fork, Woodville, 23.

38.1 Cross Front st., Fremont; thru Clyde 45.2.

45.8 Half left after railroad on Maple st.

56.6 Left at end; right at banks, Castalia, 57.1.

57.3 Half left at pond; into Washington st.

64.0 Right on Columbus ave. at park, Sandusky.

64.6 Left on Scott st.; right on McKinley 65.6.

73.9 Right-left; right at end, Huron, on Main.

74.2 Left on Van Rensselaer; past Ruggles Beach 79.

84.8 Left at end; next right at trolley, Vermilion.

95.8 Cross Broadway, Lorain; left fork 107.3.

112.3 Right on Clague road; left, Detroit road 113.1.

115.9 Right at Ridgeway; next left over Rocky river.

116.1 Left with trolley, curving left and right into Lake road; curve right on Lake ave.; thru Edgewater Park.

123.2 Right; sharp left across Superior st. viaduct. 


\section{Prescott to Ottawa, Canada, 59.2 Miles}

0.0 From King st., Prescott, northwest on Edward st., thru Spencerville 10.5 .

16.7 Left; right at Kemptville 23.9.

25.1 Left at end; next right 25.4.

28.7 Left with wires; right at end 33.6.

34.1 Left at school, Ridge Mills; right 42.2 .

45.4 Right after bridge; left at cemetery, Greeley, 45.8 .

59.2 Right with trolley on Sparks st., Ottawa.

\section{THE RUSSELL HOTEL Ottawa, Canada}

Tourists trade particularly catered to American and European Plans

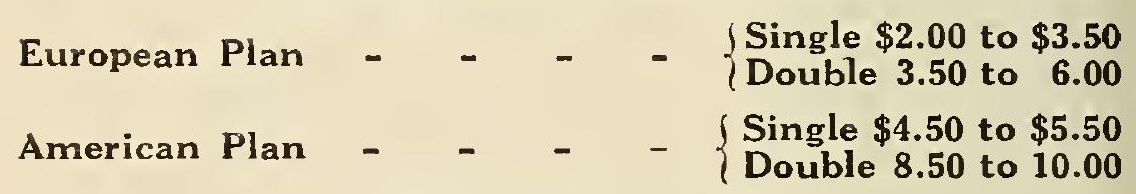

THREE DINING ROOMS

American Plan-Grill Room (a la carte)-and Ottawa's most popular Cafeteria

Garage one minute from the Hotel

Ottawa to Prescott, Canada, 59.2 Miles

0.0 From Sparks st., Ottawa, south on Bank st. with trolley.

13.4 Right fork at Greeley; right at end 13.5.

13.9 Left over bridge; left at end 16.8 .

25.1 Right at end, Ridge Mills; left 25.6.

30.5 Right; left at end 33.7; right 34.1.

35.3 Left at end, Kemptville; right at end 42.5.

48.7 Half right at Spencerville; into Edward st.

59.2 Edward st., Prescott, at King st.

\section{Che Chateau Caurier otTawa, onT.}

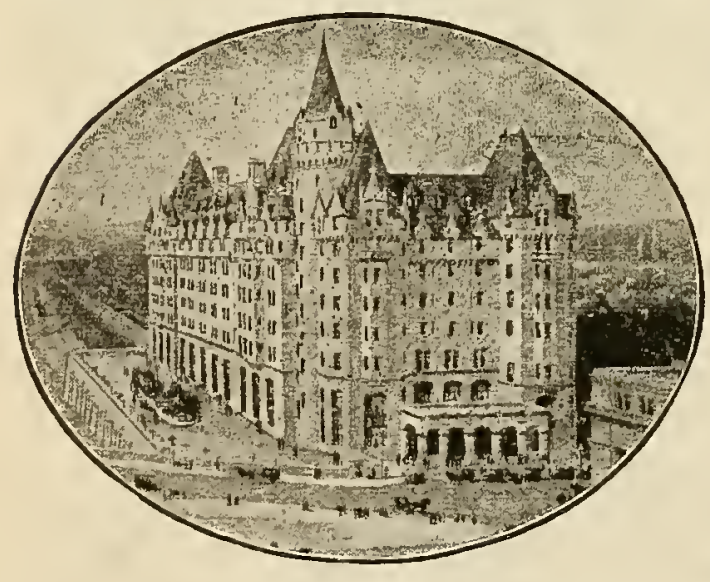

One of the finest hotels on the

American

Continent

350 Rooms

European Plan

Rates $\$ 3.50$ and up

Angus Gordon

Resident Manager

\section{TO KEEP OUT OF TROUBLE}

Use chains when pavements are slippery.

Test your brakes frequently.

Don't use glaring headlights in the streets of the city.

When approaching an automobile at night, coming in an opposite direction, slow down and dim your headlights. 
Detroit, Mich., to London, Ont., 142.7 Miles

0.0 From Grand Circus southeast on Woodward ave.

0.8 Crossferry to Windsor, Ont. Straight on.

2.4 Left from trolley; right at trolley, 2.9.

7.5 Left; thru Maidstone 13.5; Essex 17.8 .

28.3 Right at end, Olinda; left 28.9; next right.

30.4 Left at Ruthven; left 33.1; thru Leamington 34.3 ; Wheatley 31.7.

45.9 Right; thru Port Alma 55; right at end 58.3; next left thru Blenheim 72 on Talbot st.

81.4 Left, Erie st.; right at end, Ridgetown 83.9.

87.2 Left at end; right 88.1; thru Highgate 90.

92.5 Left; right at end 93.1 ; left 93.5 right 95.5.

96.8 Left at end; right at Clachan 97.7; left 103.

105.4 Right at Wardsville; thru Melbourne 120.4.

127.4 Left fork; thru Delaware 129.8; left fork 135.

136.8 Half left with trolley on Wharncliffe road.

142.1 Right after underpass on Stanley st.

142.7 Left on Riclimond st., London, Ont.

London to Detroit, Mich., 142.7 Miles

0.0 From Richmond st., London west on York st.

0.7 Left inder RR; thrı Lambeth 6.3 follow poles.

12.9 Half left at Delaware; thru Melbourne 22.3.

37.3 Left at Wardsville over bridge; right 39.7 .

45.0 Left at end, Clachan; right 45.9.

50.2 Right; thru Highgate 52.7; left 54.6; right 55.5.

55.7 Right; into Ridgetown; left on Erie 59.

61.3 Right at end; thru Talbot st., Blenheim 71.

84.3 Right, next left; thru Port Alma 87.7; Wheatley 101; Leamington 108.4 on Talbot st.

112.3 Right at end, Ruthven; left-right 113.8 .

114.4 Left at edge of Olinda; join trolley, thru Essex 125 ; and Maidstone 129.

135.2 Right with wires; left from trolley 139.8.

135.6 Right at trolley on Ouellette ave., Windsor, to ferry.

141.9 Cross ferry, straight up Woodward ave. to Grand Circus 142.7, Detroit, Mich.

Cleveland to Roledo, Ohio, 124.1 Miles

0.0 From square, Cleveland, soutluwest on Superior st., curving right 0.3 over viaduct.

0.4 Sharp right at end of bridge; left into Boulevard; thru Edgewater Park, coning into Lake ave.; keep right 3.4 .

7.9 Right with trolley, over Rocky river bridge.

8.1 Half right, next left at Ridgeway on Detroit road.

11.0 Right on Clague road; left after trolley 11.8 into Erie ave., Lorain; cross Broadway 28.3 .

39.0 Cross Main st., Vermilion; left at sign 39.2 .

39.3 Next right before railroad; past Ruggles Beach.

49.9 Right on Main st., Huron; left around postoffice.

50.2 Right-left at church; keep right on concrete 59.1.

58.0 Right on McKinley; left on Fifth into Scott st.

59.5 Right on Columbus ave. to park, Sandusky.

60.1 Left on Washington; trolley leaves 62.2.

67.0 Left at end, Castalia; right 67.5 .

78.2 Half right at end on State st., Clyde.

86.0 Cross Front st., Fremont, on State st.; cross long bridge at Woodville 101.

101.4 Half right from trolley thris Genoa 106.

106.9 Left at six corners; right at cross road 109.8.

110.7 Left at cross road, thru Latchie 112.7.

115.7 Right after railroad; into East Broadivay.

122.6 Left with cross trolley on Starr; half right on Main st. witl trolley over Maumee bridge.

123.7 Left on St. Clair to Jefferson st., Toledo, 124.1.

Kindly mention MOTOR TOURS in writing advertisers 


\section{Detroit to Kalamaz00, Mich., 142.9 Miles}

0.0 From Woodward ave., Detroit, northwest on Michigan ave., thru Dearborn 10.5; Wayne 18 ; Denton 26.

30.6 Half left with trolley on Michigan ave., Ypsilanti, thru Saline 40.5 .

41.2 Right; thru Bridgewater 47.

51.1 Left at end; right at end 54.5.

54.7 Left at end Manchester, thru Napoleon 66.

75.7 Right on Francis st.; left on Main st., Jackson 77.2.

77.7 Right from trolley at monument; thru Parma 88.

95.1 Half right on Michigan ave.; right on Superior st., Albion 95.9.

96.1 Left on Austin ave.; thru Marshall 107.

119.6 Cross Jefferson st., Battle Creek, and river 120.

120.5 Left on Kendall st.; right after bridge 120.7.

121.1 Left-right along railroad.

124.6 Right after railioad; right 127.4.

128.6 Right at end thru Augusta 129.

133.7 Half left; next right into Galesburg.

134.1 Right at monument; left across railroad 141.8 on Main st.

142.9 Court House, Kalamazoo.

\section{Kalamazoo to Chicago, Ill., 162.5 Miles}

0.0 From Court House Kalamazoo, west on Main st.

6.5 Half left; left at cemetery 8.9.

16.9 Right at school; thru Pawpaw 18.

21.3 Right; left 21.7 ; left at end 26.6 ; thru Lawrence 27 ; Hartford 33 ; Watervliet 38.

44.7 Left; left at end Riverside 45.

50.9 Right; left at postoffice Benton Harbor on Water st.

52.7 Right on Ship st., St. Joseph.

53.0 Left on State st.; left-right 53.7 ; thru Stevensville 60.

64.6 Right at Baroda; second left; next right.

65.2 Left at end; thru Galien 75.7.

76.0 Right after Culvert; thru outskirts of Three Oaks 81.5.

91.1 Right at end, thru Grand Beach 92.

98.3 Left on Franklin st., Michigan City.

100.1 Right on Ear1 road; left at end 102.3; right 102.7.

103.8 Half right; left at school 104.1; right 106.3 .

111.4 Left at sign; right from railroad bridge 111.8 .

113.8 Left at cross road; cross railroad at Porter 114.8.

115.2 Right along railroad; left across tracks 118.8.

119.4 Right at cross road; left at end 120.8 .

121.5 Right at cross road; right at cemetery 122.7.

123.7 Right fork; right at end; curving left.

124.7 Right at East Gary around brick store.

127.5 Left across railroad at Miller; next right trolley.

131.3 Straight thru Gary on Fifth ave.

134.4 Left at end; left after railroad 135.4.

138.7 Left at trolley, thru Calumet 139 on Chicago ave.

140.3 Right, Forsythe ave.; left on 145th.

141.9 Right on Calumet boulevard; cross marshes.

145.7 Half left, Indianapolis ave.; half right 147.8.

148.6 Half left at five corners on 95th; half right South Chicago ave. 149.

151.3 Half right on Jeffrey ave.; enter Jackson Park; keep left; bear right at triangle; keep left around golf grounds; curve right at three corners; then left into Midway; enter Washington Park; keep left; curve right at Concourse out of park on Garfield boulevard. 156.6 Right on Michigan ave. to Art Institute, Chicago 162.5.

Don't pass on the left hand side of a street car or any other vehicle which is driving along the street car tracks. 


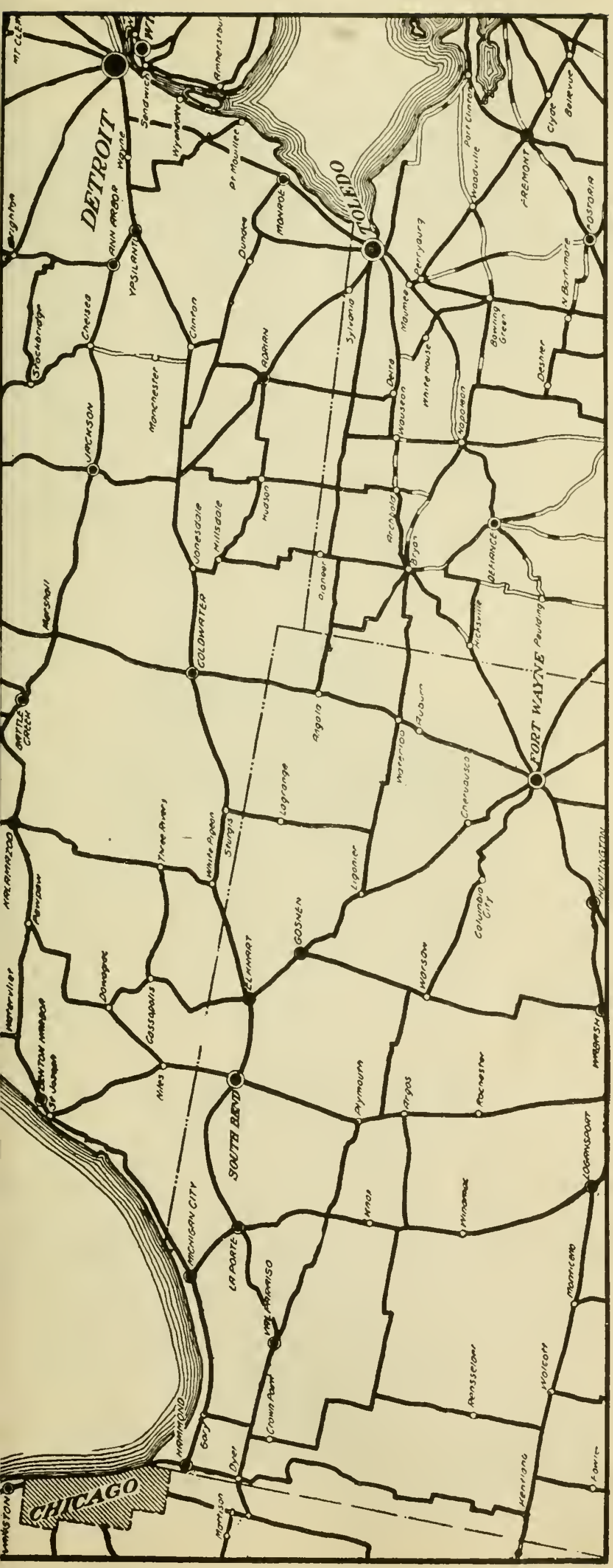


Hartford to Springfield, 26.6 Miles

0.0 From City Hall, east on Central st., curving right and left with trolley.

0.5 Right across long stone bridge.

1.7 Left with trolley at E. Hartford; under RR.

24.8 Left fork from trol.; right 25.5 on Main.

26.6 State and Main sts., Springfield, Mass.

Atlantic City to Philadelphia, Pa., 59.2 M.

0.0 From Atlantic ave., Atlantic City, north on Albany ave., becoming Verona.

4.7 Right, Main st., Pleasantville; cross RR.

7.5 Left after RR Absecon; thru Pomona 13.3 and Egg Harbor City 19.

28.2 Half right Da Costa; thru Hammonton 30.7 ; Berlin 43; Stratford 49; Magnolia 51.

56.2 Half right across RR; into Haddon ave.

58.5 Half left, Federal st., Camden to

59.2 Ferry to foot of Market st. for Philadelphia.

Philadelphia, Pa. to Atlantic Gity, N. J. Page 80

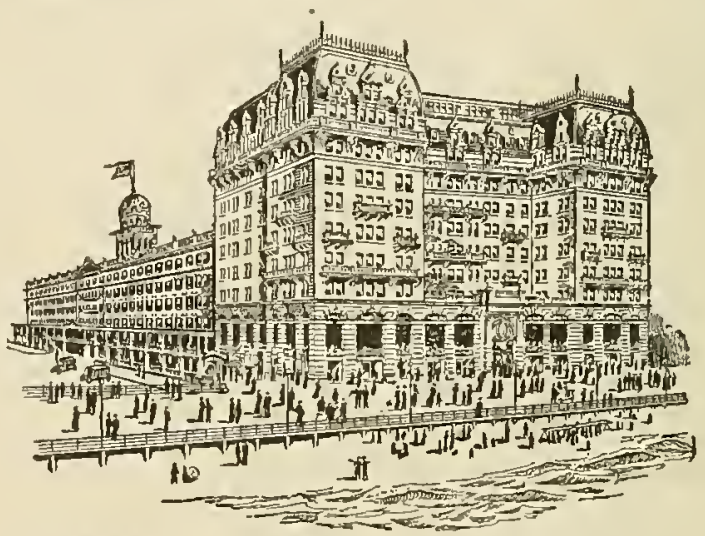

\section{The 政reakers}

Atlantic, City, New Jersey

OCEAN

FRONT American and European Plans

FIREPROOF

\section{The 将assaul}

Long Beach, Long Island AMERICAN AND EUROPEAN PLANS

Ocean Front (Excellent Cuisine)

Fireproof

\section{Our Printing Department}

Have unexcelled facilities for doing first class printing, short or long runs. Catalogs, Stationery, Maps. Will pay you to write us for estimates.

\section{MOTOR TOURS}




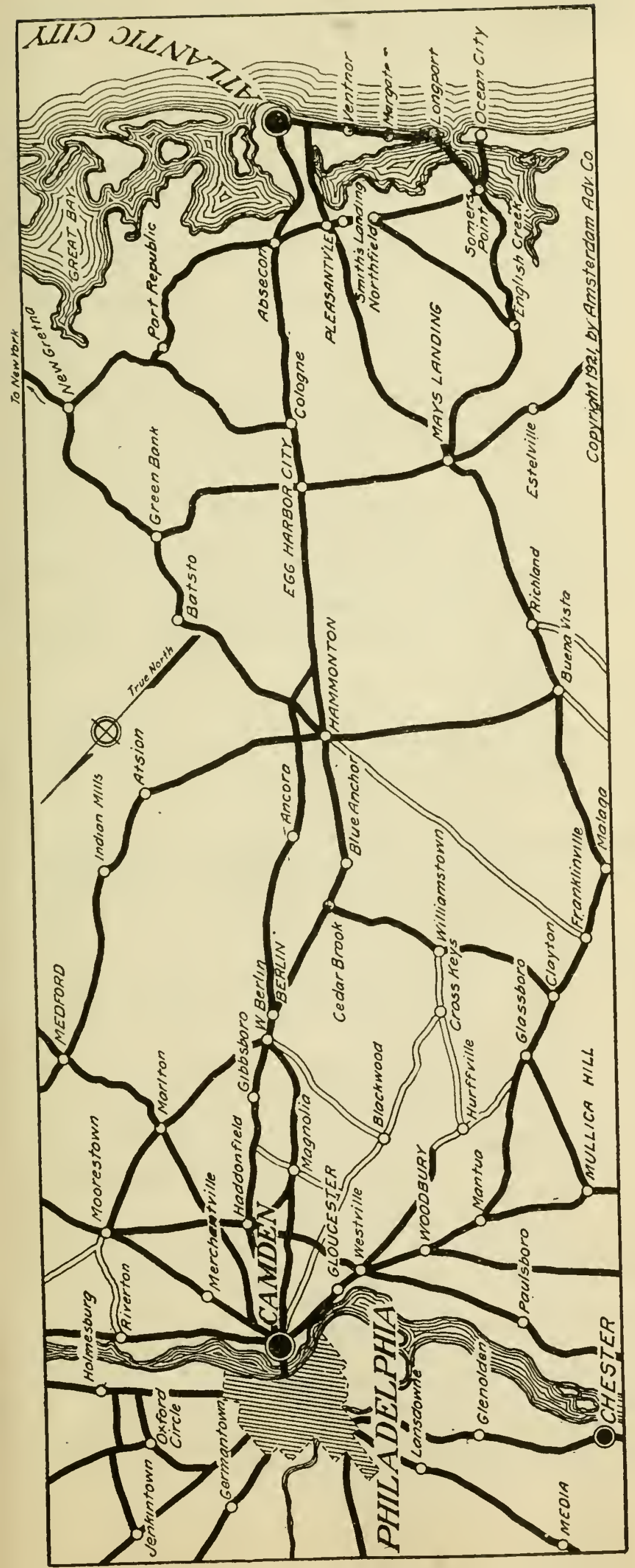


Boston to Provincetown, Mass., 131 Miles

0.0 From Massachusetts ave., Boston, west on Commonwealth, curving left 0.1 at Coilins mon. Follow parkway along Jamaica pond.

4.7 Left at 3-corners, keep left 5.6 under R.R.

5.9 Curve right and left into Morton st.

7.5 Right on Blue Hill ave., thru Mattapan 8.6.

8.9 Left with trol. on Brook road; cross R.R. at E. Milton 12 .

14.1 Left on Washington st., Quincy; thru Hingham 20.

22.8 Right on Jerusalem road; following "Cohasset" signs.

26.6 Left-right around park, Cohasset; thru $\mathbf{N}$. Scituate 28.5.

30.3 Right at Egypt; left at Greenbush 32.4.

39.6 Right fork at Marshfield; thru Kingston 48.

52.3 Plymouth. Straight ahead on Main st.; left fork 69.4.

69.6 Right across canal bridge and R.R. into Sagamore.

70.1 Left-next right past park and thru Sandwich 73.

75.8 Left fork thru W. Barnstable 80; Barnstable 85 ; Yarmouth 88.5 ; Dennis 92 ; Brewster 98.

103.5 Straight on at Orleans, joining route from Chatham.

107.5 Right at Eastham; left at end; thru S. Wellfleet 114 ; and Wellfleet 117.

131.0 Provincetown, Mass.

Provincetown to Boston, Mass., 131 Miles

0.0 Irom Provincetown, northeast, thru Truro 10; Wellfleet 14; S. Wellfleet 17 ; and $\mathbf{N}$. Eastham 21.

23.5 Right, next left at Eastham; right 25.1.

27.5 Straight on thru Orleans; Brewster 33 ; Dennis 39 ; Yarmouth 42.5; Barnstable 46; West Barnstable 51.

58.7 Left fork at monuments, Sandwich; right fork at Sagamore 60.8 .

78.7 Plymouth. Straight ahead on Court st., thru Kingston 83.

91.4 Left fork at Marshfield; right at Greenbush 98.6; left at Egypt 100.7.

104.4 Right after park, Cohasset, follow Jerusalem road to Hingham 91.

116.9 Right on Hancock st., Quincy; left fork, Adams st., 117.1 into Brook rd.

122.1 Right on Blue Hill ave., thru Mattapan.

123.5 Left at real estate office on Morton st.

125.1 Right and left curve under " $L$ " and R.R.

126.4 Right at 3-corners; follow main parkway.

130.9 Right at mon. on Commonwealth ave. to Massachusetts ave., Boston 131. 
Syracuse to Alexandria Bay, N. Y., 100.4 M.

0.0 From square, Syracuse, north on Salina st.; left 1.3 .

1.6 Right on Wolf st.; thru Cicero 10 ; Brewerton 14; Hastings 24 ; Maple View 29.5.

37.3 Left at end across bridge; next sharp right, at park, thru Pulaski 37.5 and Pierrepont Manor 50.

50.3 Right at bridge; right at three corners 53.3.

55.8 Left at 4-corners on Main St., Adams.

59.7 Right fork at P. O. Adams Center; left 67.7.

70.0 Half left on Court st., at square, Watertown. Curve right over bridge Black River on Leray st.

72.2 Half left on macadam, thru Pamelia 79.

87.8 Left at end on Main st., Theresa.

88.7 Left on Commercial st.; right at school 90.1; thru Plessis 94.4.

96.4 Left at cross road into James st.

100.4 Alexandria Bay at Church st.

\title{
1000 ISLAND HOUSE ALEXANDRIA BAY NEW YORK
}

A modern hotel of peculiar excellence and noted for years as the best cuisine and service in Northern New York : : :

POLO

\section{MOTOR BOATING} FINE FISHING

\author{
Bathing in our Casino Pool \\ Fireproof Garage \\ Send for booklet
}

\section{Crossmon House}

ALEXANDRIA BAY, N. Y.

IN THE HEART OF THE THOUSAND ISLANDS

The two largest and best Hotels on the St. Lawrence River Open May 30 to October

W. H. WARBURTON, Proprietor THE OAKS, BARTOW, FLORIDA Open all year

\section{ISLAND GARAGE}

24 Hour Service

The largest and most Modern Garage at 1000 Islands. The first as you enter Alexandria Bay on the Roosevelt Highway. HUTCHINSON BROS. Prop.

Alexandria Bay, N. Y. 


\section{Binghamton to Scranton, Pa., 63.7 Miles}

0.0 From City Hall, Binghamton, east on Court st.; keep right 4.9, thru Hallstead 15.2; New Milford 21.2.

21.7 Right; next left after railroad; left 24.8 .

25.9 Right after railroad, Heart Lake; half left 28.7.

37.1 Right at end, Foster; left fork 38.7.

43.6 Right, Nicholson; left after bridge 44.6.

48.8 Right fork at Factoryville; right 50.5.

52.5 Left-right, Dalton; thru Clarks Summit 56.

59.8 Left at end, Market st.; right on Main ave.

61.3 Left, Green Ridge st.; cross Lackawanna river.

62.0 Right, Sanderson ave.; left, Larch st.; right on Wyoming to

63.7 Linden st. at postoffice Scranton, $\mathrm{Pa}$.

\section{Scranton to Binghamton, 63.7 Miles}

0.0 From Linden st., Scranton, northeast on Wyoming.

1.1 Left, Larch st., curve right, Sanderson ave.

1.6 Left on Greenwich st.; cross Lackawanna river.

2.4 Right on North Main ave., Providence; left on Market 2.8; thru Clarks Summit 7.8.

11.2 Left-right at Dalton; left at end 13.2.

18.9 Left over bridge; right 19.2 to Nicholson.

20.2 Left, Cross st.; right fork 20.6; thru Foster 26.

31.8 Right; left across railroad at Heart Lake 38.

39.0 Right at end; left 42; thru New Milford 42.5.

46.2 Right fork, thru Hallstead 48.5; Great Bend 49.

59.0 Left fork, Court st. to City Hall, Binghamton; 63.7.

\section{TOURISTS REQUIRE NO BOND}

It is not now necessary for American tourists to obtain a custom bond before entering Canada, provided the entry is for pleasure purposes only, and not over ten days. All that is required is that an application be made at the Collector of the Port of Entry for a Ten-Day Permit to tour Canada.

If a longer stay than ten days is planned and not more than six months, a bond for double the value of the Car must be given. A bond may be obtained at Port of Entry upon payment of a fee of $\$ 5.00-$ no other expenses necessary. This bond is only required when the visit in Canada exceeds ten days.

\section{TIMELY SUGGESTIONS}

Never under any circumstances drive fast when approaching or passing a school.

When unable to see what is ahead of you at any time, slow down.

Upon a signal from another driver approaching from the rear at a reasonable speed, pull over to the extreme right and allow him to pass. The law compels you-courtesy demands it.

Spasmodic spurts of speed are dangerous.

\section{The Sign of a Good Printer}

Believe in signs?

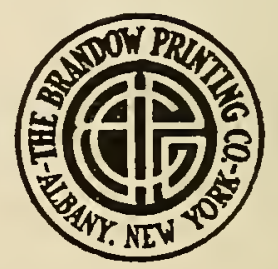


Detroit, Mich., to Toledo, 0., 59.4 Miles

0.0 From Woodward ave., Detroit, southwest on Lafayette blyd.; jog left-right 2.4-2.6.

3.3 Left, Military road; right at end, Jefferson ave.

13.4 Right fork with trolley thru Trenton 15.9.

16.2 Right; left with concrete 16.4 past monument 19.7.

21.5 Right on River road; left at Rockwood 23.5.

Continued on page 132 .

\section{Kalamazoo to Detroit, Mich., 142.9 Miles}

0.0 From Court House, Kalamazoo, east on Main.

0.3 Left fork; half right 0.9 .

8.8 Left at monument, Galesburg.

9.2 Left across railroad; next right thru Augusta 14.

14.4 Left; left at hospital Camp Custer 15.6.

18.1 Left across railroad; right fork 20.1.

21.8 Left-right; left on Kendall st. 22.2.

22.4 Right on Main st., Battle Creek.

23.6 Half left on Marshall st.; right fork from trolley 24.6 thru Marshall 36.

46.8 Right at end; left on Michigan ave., Albion 47.1.

47.8 Half left; thru Parma 55.

65.2 Left fork at monument, Jackson.

65.6 Left on Francis st.; left 57.2, thru Napoleon 77.

88.2 Right at Manchester; left 88.4 .

91.8 Right; left at end 101.8, thru Saline 102.4.

112.3 Half right with trolley, Ypsilanti; thru Denton 117; Wayne 125; Dearborn 132.4.

142.9 Detroit at Woodward ave.

\section{Chicago to Kalamazoo, 162.5 Miles}

0.0 Art Institute, Chicago, south on Michigan.

5.9 Left, Garfield boulevard; into Washington Park 6.4; curve left-right along lake: left at end into Midway: enter Jackson Park; left fork 8.6 and keep right; right at three corners 9.2 into Jeffrey ave.

11.2 Half left, South Chicago ave.; left, Ninety-fifth st. 13.5.

13.9 Half right at five corners on Ewing ave.

14.7 Half left with trolley, Indianapolis ave.

17.0 Half right Calumet bonlevard across marshes.

20.8 Left on Gostlin: right on Forsyth ave. 22.2.

22.4 Left on Chicago ave. thru Calumet 23.4 .

24.0 Right from trolley; right across railroad 27.3.

28.3 Right into Fifth ave. thru Gary 31.4.

35.2 Left across railroad at Miller; next right.

38.0 Left around store at East Gary; left fork 39.

40.0 Left at cemetery; left at cross road 41.2 .

41.9 Right; left at cross road 43.3.

43.9 Right after railroad; left from tracks 47.5 .

48.9 Right at cross road; left from railroad bridge 51 .

56.4 Left at end; right at end 58.6; right 59.2.

60.0 Left at end; right along railroad 60.4 .

62.7 Left on Franklin st., Michigan City.

64.2 Right on Second st.; thru Grand Beach 70 and outskirts Three Oaks 81 .

86.7 Left over culvert thru Galien.

97.5 Right; left at church; next right 97.9 ; left at end Baroda station 98 ; thru Stevensville 102.4.

108.8 Left-right on State st., St. Joseph.

109.5 Right on Ship st.; left at end 109.8.

111.1 Left at trolley at Water st.; right at postoffice Benton Harbor.

111.6 Left on Paw Paw ave.; right at Riverside 117.4.

117.7 Right at cross road; thru Coloma 121.7; Watervliet 124 ; and Hartford 129.

135.8 Half right; right at end 140.8 .

141.1 Left; left fork from Main st., Paw Paw 144.2.

145.4 Left at school; right-left 153.5.

162.5 Kalamazoo, Main st. at Court House. 
Toledo, O., to South Bend, Ind., 153.7 Miles

0.0 From St. Clair st., Toledo, northwest on Jefferson; bear right on Collingwood ave. 1.1.

1.5 Left on Bancroft; half right at five corners 5.

6.6 Left at end; thru Charaghar 20; Oakshade.

36.3 Right; half left 36.9 thru Fayette 42.

43.7 Left at end; right at cross road 44.2.

48.1 Left at Alvordton; right at cross road 50.3.

68.7 Left at Columbia; right at end 70; thru Metz 72.3 and Angola on Maumee st. 81.

87.1 Half right; left at end, Flint 88.4.

88.5 Right at school; left at end 90; right 90.3 .

103.5 Half left on Howpatch ave., La Grange.

104.6 Right; left at cross road 116.5.

117.9 Right at school; right fork Lincoln ave. 127.

127.4 Right at Court House on Main st., Goshen.

Continued at bottom of page 133

South Bend, Ind., to Toledo, Ohio, 153.7 M.

0.0 Main st., South Bend, east on Jefferson.

0.8 Right on Eddy st.; left on Mishawaka 1.4.

4.3 Right on Main st., Mishawaka; left on Second st.

10.0 Right across railroad at Osceola; at once left.

10.5 Left at church; right 11.8.

15.3 Half left at trolley on Franklin st.

15.8 Half right on Harrison st., Elkhart.

16.6 Right on Main st.

25.8 Half left over bridge on Pike st., Goshen.

26.2 Right, Main st.; second left, Lincoln ave.

35.8 Left at end; right at cross road 37.2.

49.1 Left at end; half right Howpatch ave. 49.5.

50.2 Half left on Central ave., La Grange.

63.4 Left at end; next right 63.7.

65.2 Left at end, Flint; right around store 65.3.

72.7 Thru Angola on Maumce st.; right fork 74.3 thru Metz, Ind., 82.3.

83.7 Left at house; right at bank, Columbia, 85.

103.4 Left at cross road; right at Allvordton 105.6.

109.4 Left at four corners; right at trolley 110 thru Fayette 111.7 ; bear right over bridge 116.8 .

117.4 Left at cross road; thru Charaghar 133.7.

147.1 Half right; half left 148.8, Bancroft st.

152.3 Right from trollcy, Collingwood ave.

152.6 Half left on Jefferson to St. Clair st.

153.7 Toledo, St. Clair and Jefferson sts.

\section{Toledo, O., to Detroit, Mich., 59.4 Miles}

0.0 St. Clair st., Toledo, northwest on Jefferson, bear right on Collingwood ave. 1.1.

3.0 Half right on Detroit ave; thru Erie 10.8 .

20.8 Cross Front st., Monroe; right on Elm ave.

21.9 Left from trolley; thru Oldport 29.8; left 31.2.

35.9 Right at end, Rockwood; left 37.9.

43.0 Right after cemetery; next left with trolley 43.2 thru Wyandotte 47.9 ; River Rouge.

55.6 Left at old Ft. Wayne on Military road.

56.1 Right on Lafayette; jog right-left 56.3.

59.4 Detroit, Cadillac square.

For Buffalo via London, Ont., see p. 112, 114, 123.

\section{Detroit to Toledo, 59.4 Miles}

Continued from page 131

28.2 Right, thru Oldport 29.6; right at trolley 37.5 .

38.4 Left on Monroe; cross Front st., Monroe, 38.6 ; thru Erie 48.6 ; into Detroit ave.

56.4 Half left on Collingwood ave. with trolley.

58.3 Half left; Jefferson ave.; The Secor 59.3.

59.4 Toledo, Jeffersen st. at St. Clair. 
Chicago, Ill., to South Bend, Ind., 98.2 Miles

0.0 From Art Institute, Chicago, south on Michigan ave.

5.9 Left on Garfield boulevard; into Washington park 6.4; curve left-right along lake; left at end into Midway; enter Jackson Park; left at fork 8.6 and keep right; right at three corners 9.2 into Jeffrey ave.

11.2 Half left on S. Chicago ave.; left on 95th st. 13.5.

13.9 Half right at five corners on Ewing ave.

14.7 Half left with trolley, Indianapolis ave.

17.0 Half right Calumet boulevard across marshes.

20.8 Left on Gostlin ; right on Forsythe 22.2.

22.4 Left on Chicago ave. thru Calumet 23.4.

24.0 Right from trolley; right across railroad 27.3.

28.3 Right into Fifth ave., thru Gary 31.4.

35.2 Left across railroad at Miller, next right.

38.0 Left around store at East Gary; left fork 39.

40.0 Left at cemetery; left at cross road 41.2.

41.9 Right; left at cross road 43.3.

43.9 Right after railroad; left from tracks 47.5.

48.9 Right at cross road; left from railroad bridge 51.

56.4 Left at end; right at end 58.6; right 59.2.

60.0 Left at end; right along railroad 60.4.

62.7 Left on Wabaslı ave.; right, Baltimore ave. 63.5.

63.7 Thru Michigan City; Rolling Prairie 79.

98.0 Bear left on Lasalle ave.; right on Main st.

98.4 Jefferson st., South Bend.

\section{South Bend, Ind., to Chicago, Ill., 98.2 Miles}

0.0 Jefferson st., South Bend, north on Main.

0.3 Left Lasalle ave.; lialf right Michigan 0.4.

17.1 Right fork (The Booljack); leaving Lincoln Way; left fork at bookjack is via Valparaiso; thru Rolling Prairie 19.7; right fork 24.9.

34.4 Half left, Ballimore st., Michigan City.

34.9 Left from trolley on Wabash; right, Earl road 35.8.

38.0 Left at end; right 38.4 ; left at end 39.2 .

39.5 Half right; left at scliool 39.8 ; right 42.

47.1 Left at sigu; right from railload bridge 47.5.

49.5 Left at cross road; cross railroad at Porter 50.5 .

50.9 Right along railroad; left across tracks 54.5.

55.1 Right at cross road; left at end 56.5.

57.2 Right at cross roal; right at cemetery 58.4.

59.4 Right fork; right at end; curving left.

60.4 Right at East Gary around brick store.

63.2 Left across railroal at Miller; next right trolley.

67.0 Straight thru Gary on Fifth ave.

70.1 Left at end; left after railroad 71.1 .

74.4 Left at trolley, thru Calumet 75 , Chicago ave.

76.0 Right, Forsythe ave.; left after railroad on 145th.

$77.6 \mathrm{R}$. on Calumet blvd.; cross marshes of Lake George.

81.4 Half left, Indiananolis ave. lialf right 83.5.

84.3 Half left at five corners on 95 th; half right South Chicago ave. 84.7 .

87.0 Half right on Jelfrey ave.; enter Jackson Park 89; keep left; bear right at triangle; keep left around golf grounds; curve right at three corners, then left into Midway; cuter Washington Park, keep left; curve right at Concourse onl of park on Garfield boulevard.

92.3 Right on Michigan ave. to Art Institute, Chicago, 98.2.

Toledo, O., to South Bend, Ind. Continued from 127.5 Second left, Pike st.; lialf right after bridge.

137.1 Left after railroad on Harrison st., Elkhart.

137.9 Half left, Franklin st.; half right from trolley 138.4; left at end 142.

143.2 Right at end; right across railroad; next left.

149.1 Right with trolley on Main st., Mishawaka.

149.4 Left aiter bridge on Mishawaka ave.

152.3 Right, Eddy st.; left, Jefferson boulevard 153.

153.7 Main st., South Bend. 
Altoona to Williamsport, Pa., 103.3 Miles

0.0 From Eleventh st., Altoona, northeast on Chestnut.

2.0 Right with trolley, Juniata; left after railroad 2.2.

7.3 Right from trolley at church, Bellwood; left 7.6.

7.9 Left over bridge; right at end 10.1.

14.4 Right with trolley on Tenth st., Tyrone.

14.7 Left from trolley on Pennsylvania; thru Port Matilda 26.3 ; and Unionville 40.3 .

45.2 Half right across railroad; right at end 45.8.

45.9 Half right, Milesburg; right, Allegheny st. 48.3.

48.7 Left, Bishop st.; left 49.7; thru Zion 53.6.

65.0 Left over bridge; left 65.3 ; right at end 65.8 .

70.4 Right fork, Cedar Springs; left fork 71.

72.4 Right, Millhall; right, Flemington 74.4 ; next left.

76.0 Left from trolley, Lock Haven; right, Water st.

82.1 Left past church; right 83.8, thru Avis.

84.6 Right; follow poles; left at end; next right 85.7 .

86.1 Left at end; right over railroad 87; left 87.1.

87.7 Right fork; left on Main st., Jersey Shore 88.4.

90.5 Left under railroad; right; thru Linden 95.8.

100.3 Left on Water st.; right on Erie ave. 100.8.

101.7 Right on Cemetery st.; left on Fourth.

103.3 Postoffice Williamsport.

\section{Williamsport to Altoona, Pa., 103.3 Miles}

0.0 From postoffice Williamsport, west on -Fourth st.

1.4 Right on Cemetery st., second left, Erie ave.

2.1 Right fork from trolley; left on Race st. 2.4.

3.0 Right, West Fourth st.; left at end 4; thru Linden 7.5.

12.8 Left under railroad; right, Allegheny st., Jersey Shore 15.

15.7 Left fork; right after trolley leaves 16.1 .

17.2 Right over bridge; left at end 17.5.

19.5 Left at end beyond Avis; right at end 21.2.

27.3 Left on Mill; right on Main st., Lock Haven.

29.0 Right, Flemington; next left; half right 30.6.

31.0 Left at end; half right, Cedar Springs 33 .

37.6 Left; right at end 38.1 ; right 38.4 ; thru Zion 49.8 .

53.7 Right at end, Bishop st.; right on Allegheny 54.7.

55.1 Left, Lyman st., Bellefonte; left, Milesburg 57.5 .

57.6 Left after bridge; left after railroad 58.2; thru Unionville 63.1; Port Matilda 75.

88.7 Right at trolley, Tenth st., Tyrone; under RR left.

93.3 Left across bridge Juaniata river; right 95.6.

95.8 Right; left, Bellwood 96.1; right at end 98.5.

101.2 Right over railroad; left on Fourth ave., Juniata.

103.3 End of Fourth ave., at postoffice Altoona.

Waverly, N. Y., to Scranton, Pa., 86.9 Miles

0.0 From Fulton st., Waverly, east on Broad st.

0.4 Right, Spalding ave. over railroad; next left.

1.8 Left, Stephenson st.; right, Elmer ave, Sayre.

3.2 Right, Pine st.; left on Main st., Athens.

4.7 Right, Tioga st. over bridge; left 5.1.

7.9 Thru Milan; Ulster 12; North Towanda 18.7.

19.9 Right, Wysox; left at Durrell 27.3.

27.4 Right; left at end 30.6 ; left at end 31.2.

31.7 Left at end; left 36.8 into Wyalusing.

44.3 Left at end over bridge; right, Laceyville.

45.2 Right-left at Skinners Eddy; right 51.1.

52.7 Right at Meshoppen; keep left at three forks.

57.4 Left at end; thru Tunkhannock 61.6.

64.7 Right over bridge; left 57 ; right at end 71.5.

73.1 Right at end, Factoryville; right 73.8.

75.8 Left-right, Dalton; thru Clarks Summit 79.

83.1 Left at end, Market st.; right on Main ave.

84.6 Left, Green Ridge st. over Lackawanna river 85.3; right, Sanderson ave.; left, Larch st.; right on Wyoming ave.

86.9 Wyoming ave. and Linden st., Scranton. 
Philadelphia to Harrisburg, Pa., 107.7 M.

0.0 From City Hall, Philadelphia, north on Broad.

0.6 Left, Spring Garden st.; bear right on Parkway; keep left at monuments 1.5 and 2 .

7.9 Left at monument; right, McCallum st. 9.1.

10.1 Right, Mermaid lane; left, St. Martin's line.

10.9 Right, Willard road; left, Seminole st. 11.1.

11.8 Right at end, Chestnut Hill ave.; left at trolley 12.3; thru Norristown 21.1; Eagleville 26.

28.7 Thru Collegeville; Trappe 30.5; Limerick 33.5.

40.3 Pottstown, cross York st.

4t.3 Left fork, Douglasville; thru Amityville 48.5.

53.5 Left fork, Reiffton; follow trolley into Penn st.

56.3 Reading, Penn square, straight thru Wernersville 64.2, Somelsdorf 70, Lebanon 83.3, and Humelstown 98.7.

107.0 Left fork, Mulberry st., from trolley into Fourth.

107.7 Left, Market st. to square, Harrisburg, 107.7.

\section{Harrisburg to Philadelphia, 107.7 Miles}

0.0 From square, Harrisburg, north on Market.

0.1 Right on Fourth st. over bridge; right at end.

9.5 Left fork from trolley; thru Palmyra 15.4; Lebanon 24.4; Myerstown 31.2; Wernersville 43.5.

15.8 Left at end to Penn square, Reading, 51.4.

52.0 Bear right with trolley; left fork 54.

63.4 Right across railroad at Douglasville; thru Pottstown 67.6 ; Limerick 74.

79.1 Right at Collegeville; thru Eagleville 81.8.

86.5 Thru Morristown; follow trolley, several turns.

93.1 Left fork with branch trolley, Barron Hill.

95.5 Right, Chestnut Hill ave.; left Seminole st. 96.

96.7 Right over railroad; left; right at end 97.1.

97.6 Left on McCallum st. over viaduct; right on Lincoln drive 98.7 into Fairmount Park 99.4.

99.8 Right at end along creek and river.

106.1 Left, Spring Garden st.; right, Broad st.

107.7 Philadelphia, City Hall.

\section{Elmira to Williamsport, Pa., 80.1 Miles}

0.0 From Water st., Elmira, south on Main.

0.6 Half right with trolley on Pennsylvania ave., thru Seely Creek 7.4 into Mosherville.

10.5 Right after bridge; left at Job's Corners 14.1.

18.7 ight after bridge; left 23.4 ; right 23.6.

29.4 Half right on Elmira st.; left on Main st., Mansfield 29.7 .

34.5 Right at Covington; left 34.7.

39.2 Right at end, Blossburg; right over bridge.

49.8 Right fork, Liberty; right at end 53.2.

65.5 Right after railroad, Trout Run; left 69 ; left 73.

77.7 Left, Erie ave.; right, Cemetery st. 78.6.

78.7 Left on Fourth st. to postoffice Williamsport 80.1 .

\section{Williamsport to Elmira, N. Y., 80.1 Miles}

0.0 From postoffice Williamsport, west on Fourth st.

1.4 Right, Cemetery st., second left, Erie ave.

2.1 Right fork from trolley; sharp right Race st. 2.4.

5.6 Left across bridge; left at end 7.1; thru Hepburnville 7.4, and Cogan Valley 8.6.

14.6 Left at Trout Run; thru Buttonwood 25.5.

26.9 Left around house; right fork at Liberty 30.3 .

35.8 Right fork; left at end 40.2 to Blossburg.

40.9 Left over bridge; left at Covington 45.8.

50.4 Right on Elmira st., Mansfield, at church.

56.5 Left at end over bridge; right at end 56.8 .

61.4 Left across bridge; right at Job's Corners 66.

69.6 Left over bridge, Mosherville; thru Seely Creek 72.7.

79.5 Left on Main to Water st., Elmira 80.1. 
Port Jervis, N. Y., to Eiaston, Pa., 64.1 Miles

0.0 From Front st., Port Jervis, southwest on Pike st.; cross toll bridge Delaware river; thru Matamoras, $\mathrm{Pa}$.

7.0 Left Harford st., Milford; second right Mott st.

12.0 Left fork; thru Dingman's 15.5 ; Bushkill 27.4.

28.1 Right fork, thru Echo Lake 31.3 ; left fork 32.5 .

36.5 Right at Shawnee; over bridge; left at end 38.6.

39.2 Left fork; left at end 39.7.

39.8 Straight thru five corners, Delaware Water Gap.

45.1 Right from trolley, Portland; left over bridge 45.4.

48.2 Right at end;-left fork; thru Richmond 51.2.

55.6 Right fork at church; thru Martin's Creek 56.5.

63.8 Left at end on North Third st. with trolley to

64.1 Easton, center square at monument.

\section{Easton to Port Jervis, N. Y., 64.1 Miles}

0.0 From square, Easton, north on Third st.

0.3 Right-on Delaware st.; thru Martin's Creek 7.6.

15.9 Left fork, thru Mount Bethel 17.5.

18.4 Left fork from trolley; right at end 18.6.

18.9 Left at trolley, Portland; thru Slateford 20.7.

24.3 Straight thru five corners, Delaware Water Gap.

24.6 Right after bridge; right across bridge 25.6.

27.6 Left fork at Shawnee-on-Delaware; thru Bushkill 36.7 and Dingman's Ferry 48.6.

56.5 Half left, Mott st.; left, Harford st. 56.9.

57.1 Right, Broad st, Milford, across bridge.

62.6 Right fork; thru Matamoras; cross river.

64.1 Port Jervis, N. Y., Pike st. at Front st.

\section{Philadelphia to Easton Pa., 70.8 Miles}

0.0 From City Hall, Philadelphia, north on Broad st.

7.4 Left, Haines st.; right, Stenton ave. 8.4.

11.9 Right fork; thru Ambler 17.

19.2 Right, Springhouse; thru Montgomeryville 23.8 .

26.8 Half left at cross road; right fork 29.3 ; thru Sellersville 33.1 .

39.7 Left, Quakertown, with trolley; thru Center Valley 47.

53.8 Left at end on Auburn st.; next right.

54.3 Right at end on Union; next left on Sixth.

54.5 Allentown, right on Hamilton st.; follow trolley into Broad st., Bethlehem, 59.7.

60.2 Left with trolley on Linden; right 60.8 .

69.0 Left at six corners; right at end 69.4.

70.8 Easton, Northampton st. at the square.

\section{Easton to Philadelphia, $\mathbf{P a}$., 70.8 Miles}

0.0 From square, Easton, west on Northampton st.

1.4 Left beyond garage; square right 1.8 .

10.0 Half left on Linden; right on Broad st., Bethlehem 10.6 into Hamilton st.

16.3 Allentown; left on Sixth st.

16.5 Right on Union; next left on Lehigh st.

17.0 Left, Auburn st.; next right over South Mountain.

20.6 Left with trolley; thru Center Valley 23.8.

31.2 Right at hotel, Quakertown, with trolley.

36.2 Right fork from trolley; thru Sellersville 37.8.

41.6 Half left; half right at cross road 44.

47.1 Right fork, Montgomeryville; left at Springhouse 51.6, thru Ambler 54.

48.9 Left fork, Stanton ave.; left at store Haines st.

53.5 Right on North Broad st. with trolley.

70.8 Philadelphia, City Hall. 


\section{Washington to Harrisburg, Pa., 122.5 Miles}

0.0 From White House, north on Jackson pl.

0.1 Bear left on Connecticut ave.

1.4 Left fork at monument; over bridge 1.6.

5.9 Left at Chevy Chase Club on Bradley lane.

6.6 Right at trolley; right fork from trolley 7.2.

14.8 Right from trolley Rockville; keep left 19.4.

31.5 Sharp left at Damascus to Ridgeville.

37.4 Left, thru New Market 43.3.

51.1 Frederick, Md.; right on Market st.; left 53.4; thru Harmony Grove 54.2.

60.7 Left at four corners; 1eft at end, Thurmont 67.

67.4 Right after railroad; right at end 68.9 .

73.6 Left over bridge; thru Emmittsburg 74.6.

81.8 Half left; Confederate ave.; right 84.6.

85.3 Gettysburg; left at square on Carlisle st.

85.7 Right at monument on Lincoln, curving left; thru edge of York Springs 99.

107.4 Right at end, Dillsburg; left at cemetery 107.6.

116.5 Left; left-right; Shiremanstown.

117.5 Right at end; left at trolley 119; next right.

122.5 Market square, Harrisburg.

Harrisburg to Washington, D. C., 122.5 M.

0.0 From square, Harrisburg, southwest on Market st. over Susquehanna bridge; curve left under railroad's.

3.6 Left with trolley; next right from track.

5.1 Left to Shiremanstown; left at end; right.

6.0 Right; left fork 8.4; thru Sheperdstown 10.

14.9 Right at cemetery, Dillsburg; second left 15.1.

15.3 Half right; thru edge of York Springs 23.8.

36.8 Left at monument on Carlisle to square.

37.2 Gettysburg, right on Chambersburg st.

37.9 Left, Confederate ave.; half right 40.7 .

47.9 Thru Emmittsburg; right at four corners 48.4.

53.6 Half left at school; left under railroad 55.2.

55.5 Right at Thurmont; right at four corners 61.8.

62.5 Right fork; thru Harmony Grove 68.3.

71.4 Frederick, left on Patrick st. to Ridgeville.

85.0 Right at sign; sharp right, danger 91.

107.7 Right at trolley, Rockville; cross railroad.

115.9 Left, Bradley lane; right Connecticut ave. 116.6.

122.5 Washington, D. C., Lafayette Park.

Harrisburg to Williamsport, Pa., 93.6 Miles

0.0 Market st., Harrisburg, north on Front.

14.4 Left over long bridge Susquehanna river.

16.4 Right; thru New Buffalo 19.5; Liverpool 29.

35.2 Right over bridge; thru Selinsgrove 47.7.

48.5 Right across Penn's Creek bridge.

48.8 Right fork; thru edge of Sunbury 52.4.

54.4 Right over river bridge into Northumberland.

54.9 Left, Duke st.; right fork, Lewisburg 62.2.

67.8 Right under railroad from trolley; thri Muncy 80.

81.1 Left after covered bridge; thru Montoursville 89.

93.6 Williamsport, Pine and Third sts.

Williamsport to Harrisburg, Pa., 93.6 Miles

0.0 From Pine st., Williamsport, northeast on Third with trolley, thru Montoursville 4.5 .

12.1 Right over covered bridge; thru Muncy 13.6.

19.0 Right fork; left 25.8 ; thru Lewisburg 31.4.

38.7 Right at end, Water st., Northumberland; cross Susquehanna river; thru edge of Sunbury 41.2.

45.0 Right over Penn's Creek; left at end; thru Selinsgrove 45.9 ; McKee's Half Falls 56.6.

58.4 Left at end after bridge; thru Liverpool 64.6.

77.2 Left at end; left over Susquehanna river 78.8 .

79.2 Right at Clark's Ferry; thru Dauphin 85.

93.6 Market st., Harrisburg. 


\section{Pittsburgh to Erie, Pa., 131.6 Miles}

0.0 From Oliver ave., Pittsburgh, west on Liberty, at once right on Federal st. over Allegheny river; follow trolley thru West View 7.3.

8.0 Right fork; thru Perrysville 9; keep left 9.4 thru Wexford 16.

29.6 Right between church and school, Zelienople.

30.5 Right at Harmony; thru Middle Lancaster 33.7; Portersville 39.5 and Harlansburg 47.

47.3 Right fork, thru Leesburg 55.

61.5 Straight, past C. H., Mercer; right fork 61.7

67.2 Left fork, follow poles thru Sheakleyville 76.2 and Custards 85.4 ; bear left 84.5 .

90.0 Right fork from trolley.

91.2 Left after railroad on Water st.

91.8 Right, Chestnut st., Meadville; left, Park ave. 91.8.

92.3 Left on Baldwin st.; right 96.2.

97.8 Left at trolley thru Saegerstown.

102.6 Right at Wenango with trolley; left at end 104; thru Cambridge Springs 106.5 and Waterford 116.7 .

117.2 Left fork; right fork 117.7 .

127.5 Right at cross road keep right, State st.

131.6 Erie, Pa., at Park.

\section{Erie to Pittsburgh, Pa., 131.6 Miles}

0.0 From Park, Erie, south on State st.

3.1 Left fork; left at cross road 4.1.

14.1 Half left thru Waterford 14.8; right forks 15.8-19.8.

25.1 Right fork after bridge, into Venango ave.

27.6 Right across trolley and railroad; left at Venango 39.

31.9 Right across railroad; thru Saegerstown 33.5.

33.8 Right at end over bridge; left at end 34.8 .

39.3 Right, Park ave; right, Chestnut st. 39.9.

40.1 Left, Water st., Meadville; right on Mercer 40.5.

40.9 Left fork; left fork 42.9; keep right 47.2; thru Custards 48.8; Sheakleyville 55.4; Leesburg 76.6; Harlansburg 84.6; Portersville 92.1, and Middle Lancaster 98.

101.1 Left at Harmony; right at cross road, Zelienople 102; thru Wexford 105.6; Perrysville 122.6; West View 124.3 .

126.2 Right fork with trolley; right fork 127.4 into Federal st.; cross Allegheny' river 131.2.

131.5 Left on Liberty to Oliver ave., Pittsburgh 131.6.

\section{Scranton to Waverly, N. Y., 86.9 Miles}

0.0 From Linden st., Scranton, northeast on Wyoming ave.

1.1 Left, Larch st.; curve right, Sanderson ave.

1.6 Left on Green Ridge st. over Lackawanna river.

2.4 Right, North Main ave., Providence; left on Market 2.8; thru Clarks Summit 7.8.

11.2 Left-right at Dalton; thru Factoryville 15.

15.4 Left; left, next rlght at end 19.8.

21.8 Left at end thru Tunkhonnock; right 29.6.

33.1 Right at end; left, Neshoppen 34.4.

36.0 Left at end; right, Skinners Eddy 41.8.

42.0 Left fork, thru Laceyville 42.7 ; right 42.8 .

49.6 Left at Wyalusing; right 15.2 ; left 52.3 .

59.6 Left at end over bridge next right, Burrell.

63.9 Left at Wysox; right on Main st. 66.5.

66.9 Left, York ave., Towanda; thru Milan 78.9.

82.0 Right, Tioga st.; left, South Main st., Athens.

83.6 Right, Pine st.; left, Wells ave. to Sayre.

84.6 Left, Stephenson st.; right on Wilbur ave.

86.3 Right on Spalding ave.; left on Broad st.

86.9 Waverly, Broad st. at Fulton. 
Dubois to Pittsburgh, Pa., 107 Miles

0.0 From Long ave., Dubois, southwest on Brady st.

3.1 Left over railroad, thru Salem 4; Luthersburg 5.8.

9.0 Left at monument; thru Troutville; Big Run 16.3.

19.9 Left at cross road -from trolley; thru Cloe 21.7.

23.7 Left from trolley; Punxutawney; next right.

25.4 Left at end; thru Trave City 32 .

37.6 Left, Smithsburg; thru Rural Valley 47.7.

59.7 Cross McKane st., Kittaniny, and Allegheny river.

60.5 Left at sign; left at end 60.7 ; left 68.3

68.5 Right at end Slatelick; right at Freeport 75.6 ; next left over bridge thru Compton 81.

82.4 Left, Corbett st., Tarantum; right Fifth ave.

85.9 Left at Boquet over railroad and river on $N$ inth st.

86.4 Right, Fiftl ave., New Kensington; left on Fourth st.; next right after railroad.

87.1 Left fork, Parnassus; left at end 87.3.

89.9 Right; right fork 91.6 ; left at end 92.8 .

93.1 Right, Unity; left, North Bessemer 93.4; right at end.

98.6 Right, Frankstown ave.; left on Braddock 99.9.

100.5 Right on Penn ave.; left on Witfield st.; right at end on Baum boulevard; curve right, Craig st. 104, and left, Bigelow boulevard.

106.8 Right, Oliver ave. to Smithfield st., Pittsburgh 107.

\section{Pittsburgh to Dubois, Pa., 107 Miles}

0.0 From Smithfield st., Pittsburgh, east on Oliver; curve left on Bigelow boulevard 0.2.

2.5 Curve right; keep left on Craig st. 2.7.

2.9 Left on Baum boulevard; bear left 4.4 .

4.5 Next right, Penn ave.; left on Braddock.

7.0 Right, Frankstown ave.; left fork 7.2.

8.4 Left at trolley; right fork 10.3 ; left at end.

13.5 Right at end, North Bessemer; left, Unity 13.8.

14.2 Right from railroad; left fork 15.4; right 17.1 .

19.8 Half right, Freeport st.; left at end 20.1.

20.2 Right on Fifth ave., New Kensington; left on Ninth; right at end 21.

24.0 Left fork; left, Corbett st., Tarentum.

24.5 Right at end, thru Pompton 26; right 30.7.

31.2 Left, Freeport; left fork 36.2 ; left 38.3.

38.5 Right at cemetery, Slatelick; right at end 46.4.

46.7 Left at end; cross river; thru Kittaniny 47.

61.5 Left fork at school; left at end 69.3.

69.5 Right at Smicksburg; thru Trade City 75.

81.5 Right; left at end 83.1.

83.4 Right on Mohoning st., Punxsutawney.

87.0 Right at trolley; thru Troutville. 97.

98.0 Right at end; left fork, Luthersburg 101.

107.0 Brady st. at Long ave., Dubois.

\section{Delaware Water Gap to Wilkes-Barre, Pa.,} 55.7 Miles

0.0 From park, Delaware Water Gap, northwest; bear right after bridge; right 0.7 ; curve left.

1.7 Left at sign; right, Prospect st. 3.1.

3.3 Left on Ridgeway; left fork 3.6; right with trolley on Main st. 4.7; right on Ninth st., Stroudsburg, from trolley.

9.6 Right at Bartonsville; thru Tannersville 12.

16.7 Left fork, Swiftwater; left fork 17.

20.4 Left, Pocono Summit; thru Naomi Pines 24.1.

26.2 Right at end; left fork at hotel 26.4.

32.7 Right at end thru Stoddartsville 36.1.

44.6 Right after stone bridge; right at end 45.

54.1 Left at end after railroad bridge on Kidder st.

54.6 Half right, Butler st.; left at end.

55.7 Wilkes-Barre, Main st. at Public Square. 


\section{Pittsburgh to Warren, Pa., 146 Miles}

0.0 From Smithfield st., Pittsburgh, east on Oliver.

2.5 Curve right and keep left on Craig st. 2.7.

2.9 Left on Baum boulevard; left 4.4; on Highland ave.

7.3 Left at end over bridge; next $\mathbf{R}$. over Allegheny river

7.9 Left at end on Main st., Sharpsburg; right on Eighteenth; left on Middle 8.2.

8.5 Right at end on Thirteenth; left on Clay; left on Garner; right on North Main; bear left and right on Bridge st.

9.4 Half right Butler st., Etna; thru Bakerstown 22

27.7 Left fork, Glade Mills; cross railroad M cBride 33.5 .

38.6 Left at end; next right, Etna st.; left over bridge on Center ave. 38.8.

39.0 Right on Main st., Butler, past C. H.

39.2 Right, Clay st.; left-right 39.4; half left, Brady st. 39.5 ; thru Chicora 50 ; Kaylor 55.

59.9 Right at East Brady; left 60.2; right 60.6 thru Rimersburg 69.

74.6 Right at Sligo; left 77.6 thru Reidsburg 80.5.

80.7 Left over railroad; left on Main st., Clarion.

85.9 Right at Fair Grounds ; right at church 88.8.

95.8 Right; next left thru Snydersburg; Lapeer 101.

118.9 Left at Pigeon; right at end, Blue Jay 124.2.

127.1 Right over bridge; next left; left at end 131 ; thru Sheffield 133; Tiona 137 ; Clarendon 139.6.

141.1 Right fork from poles; bear left.

146.0 Warren, Pennsylvania ave. at monument.

Warren to Pittsburgh, Pa., 146. Miles

0.0 From monument, Warren, east on Pennsylvania ave.; thru Clarendon 6.4; Tiona 9; Sheffield 11.

15.0 Right; right over bridge, next left 18.9.

21.8 Left at Blue Jay; right at end, Pigeon 27.1; thru Lapeer 45 ; Snydersburg 49.

50.2 Right at end; next left at end; right 50.4 ; next half left thru Lucinda 51.

57.2 Left; left at end, Main st., Clarion 60.

60.4 Right at Court House on Fifth st.; thru Reidsburg 65.5 .

68.4 Right; left at Sligo 71.4; thru Rimersburg 77.

85.4 Right; right at end; left at bank, East Brady 86.

86.2 Left fork across railroad; thru Kaylor 91; Chicora 96.

106.8 Left on Main st., Butler, past Court House.

107.0 Left on Wayne st. over bridge; cross railroad at McBride 112.5; thru Glade Mills 118.3; Bakerstown 124.

136.6 Half left at Etna on Bridge st.; left at trolley.

136.9 Left on Garret st., next right on Clay; right across railroad, next left 137.2.

137.8 Right on Eighteenth st.; left on Main st., Sharpsburg.

138.0 Right, cross Allegheny river; left at end; next right.

140.1 Left, Highland ave.; right Baum blvd.; bear right.

146.0 Oliver st. at Smithfield st., Pittsburgh.

\section{Wilkes-Barre to Delaware W. Gap, Pa., 55.7}

0.0 From Square, Wilkes-Barre, northeast on Main st.

0.7 Right, Butler st.; left on Kidder st. 1.1.

1.8 Right over railroad; left at end Bear Creek 11.5.

17.6 Right fork thru Stoddartsville 19.6.

23.0 Left; right fork 26.3 ; right at end 29.2.

29.5 Left; right at end 30.6 ; thru Naomi Pines 31.6 ; left at end 34.4.

35.3 Right, Pocono Summit; right at end 38.7.

43.1 Left at end; right thru Tannersville 43.8 .

46.1 Left, Bartonsville; right fork Ninth st. 50.7.

50.9 Left at end, Main st., Stoudsburg, trolley.

51.9 Right fork from trolley; left fork Ridgeway st.

52.3 Right on Prospect st.; left at end 52.5.

53.9 Right at cross road; left at end 55.

55.7 Delaware Water Gap at small park. 


\section{New York to Delaware Water Gap, 83 Miles}

0.0 Ferry, West Forty-second st. to Weehawken, N. J.

0.6 Sharg left at top, at once right on Third.

1.1 Left-right; left at end on boulevard 1.3 .

5.1 Right at trolley, Newark ave; half right 7.1.

10.9 Left at end, Belleville; second right, John st.

15.2 Montclair; bear right on Bloomfield ave.

19.0 - Right fork, Caldwell; left fork 19.7.

26.5 Left at Parsippany; left under railroad 31.

33.3 Right at park, Morristown; next right on Washington ave.; thru Mendham 40.1 .

44.7 Left at hotel; keep right, Chester 45.7.

50.1 Right over bridge and railroad, German Valley; rough grade over Schooley Mountain.

56.5 Hackettstown bear left on Main st.; right fork 57.6.

61.1 Left fork thru Denville 62.

67.9 Right at Buttzville; thru Bridgeville 69.

74.6 Left under railroad; right over Delaware river 75.6.

76.0 Right at end thru Portland, Pa., 77.8.

83.0 Delaware Water Gap, Pa., at small park.

Delawar̆e Water Gap to New York, 83 Miles

0.0 From park, Delaware Water Gap, run south with trolley thru Portland 5.2.

7.2 Left over Delaware river bridge; left at end 8.4.

8.5 Right at end, Delaware; left at end 12.2.

15.1 Left at hotel; Buttzville; left at end 20.1.

25.1 Left fork, Main st., Hackettstown 26.5; keep right Mill st.; right at monument.

28.1 Left fork over Schooley Mountain.

32.9 Left at end after railroad, German Valley.

38.3 Half right at five comers; thru Mendham.

39.6 Left at park Morristown; keep left.

51.9 Right fork under railroad; curve right 52.

56.5 Right at end Parsippany; thru Caldwell.

67.8 Bear left at brick church on Glenridge ave., Montclair, leave trolley.

72.0 Left at end Belleville; next right.

77.9 Left on Boulevarcl, leaving trolley.

81.7 Right at cemetery on Third st.; left-right 81.9.

82.4 Left at end; at once sharp right down hill.

83.0 Ferry, to West Forty-second st., Manhattan, N. Y.

\section{Delaware Water Gap to Scranton, Pa.,}

\subsection{Miles}

0.0 North from parfl Delaware Water Gap; $\mathrm{R}$ fork 0.2.

0.6 Right; left 1.7; right. Prospect st. 3.1.

3.3 Left on Ridgeway st.; left fork 3.6.

4.3 Right, Main st., Stroudsburg; right, Ninth st.

9.6 Right at Bartonsville thru Tannersville 12.

16.7 Left fork, Swiftwater; next right fork; thru Mount Pocono 19.

19.5 Straight on at three forks thru Tobyhanna 24.5 .

41.2 Left upgrade at Elmhurst; right fork on boulevard 46.5 ; left at cross road 48.

48.4 Right at end out of Park; next left on

49.6 Linden st. at Washington ave, Scranton.

Scranton to Delaware Water Gap, Pa.,

0.0 From Washington ave., Scranton, southeast on Linden.

1.1 Right-left into park; right over railroad bridge.

8.4 Right at end, Elmhurst; thru Moscow 11.7; left fork 14.1; thru Mount Pocono 30.6 and Tannersville 37.7.

40.1 Left, Bartonsville; right fork Ninth st. 44.7.

44.9 Left, Main st., Stroudsburg; R fork 45.8; L fork 46.

46.3 Right, Prospect st.; left at end; right 47.9.

49.0 Left at end, to Park, Delaware Water Gap 49.6. 


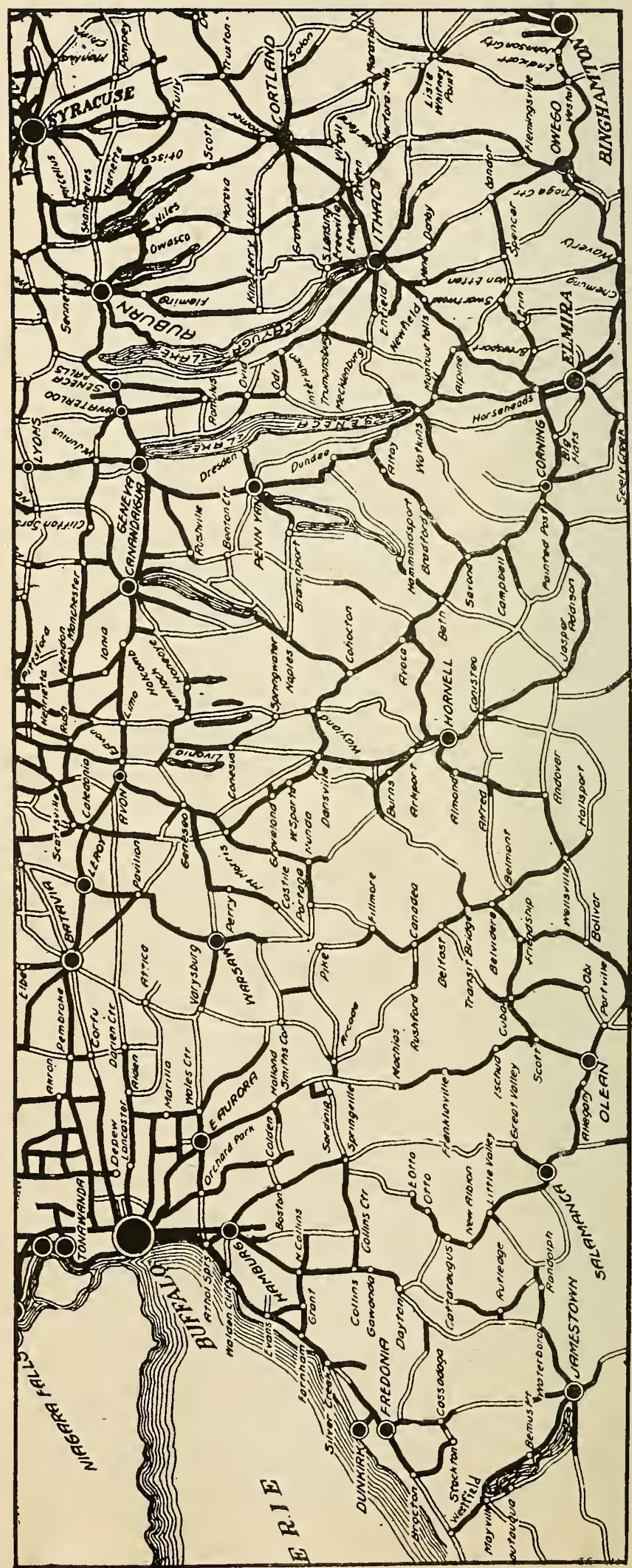




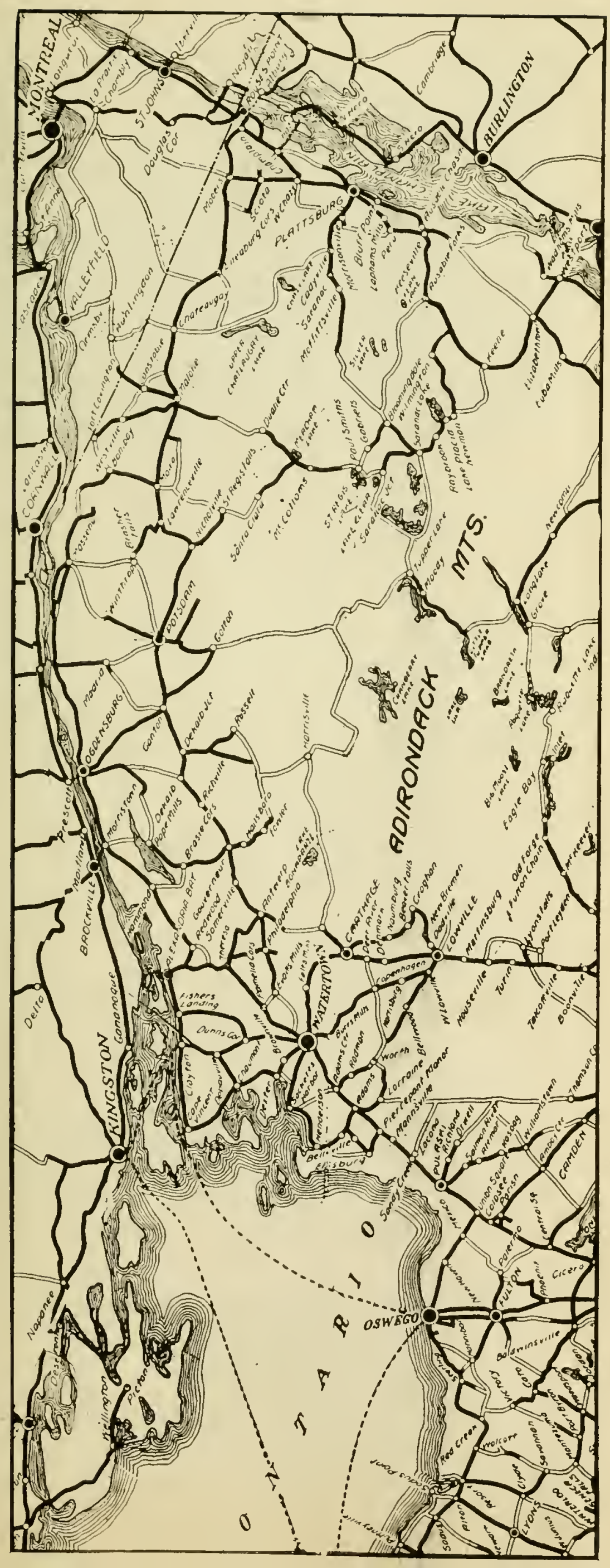




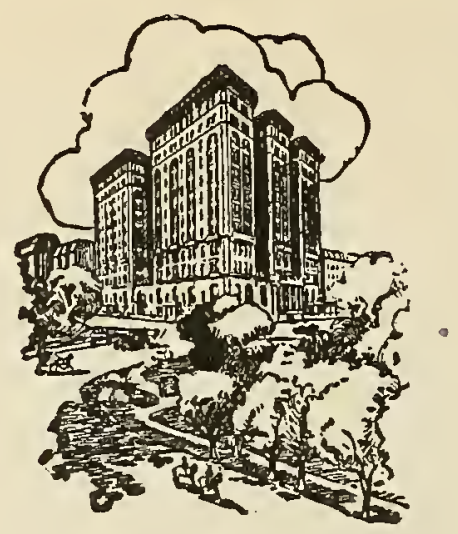

\section{A J E S T I C}

\section{HOTEL and RESTAURANTS COPELAND TOWNSEND}

Fronting Central Park at West SeventySecond Street - the motor entrance

NEW YORK

Delightful vista, yet surprisingly convenient to the heart of the great metropolis.

Accommodations and service all in consonance with the high character indicated by the name and setting and completely satisfying the expectations of its clientele.

\section{THE HURRICANE DEGK}

atop the hotel is open during the summer months. Afternoon and evening dances. Pleasing orchestral music. Excellent cuisine and service under the open sky.

\section{MARLBOROUGH HOTEL}

\section{Asbury Park, N. J.}

Seashore and Country Combined

Open the entire year

American Plan

ROOMS AND BATH

Running Hot and Cold Water in Every Room

Hotel of Service, Comfort, Quality, Courtesy and Personal

Supervision. Guests extended privileges to new 18 hole golf course

HAROLD W. SEXTON, Manager 


\section{TOURISTS}

visit

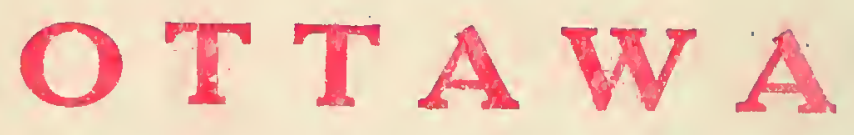

THE CANADIAN CAPITAL

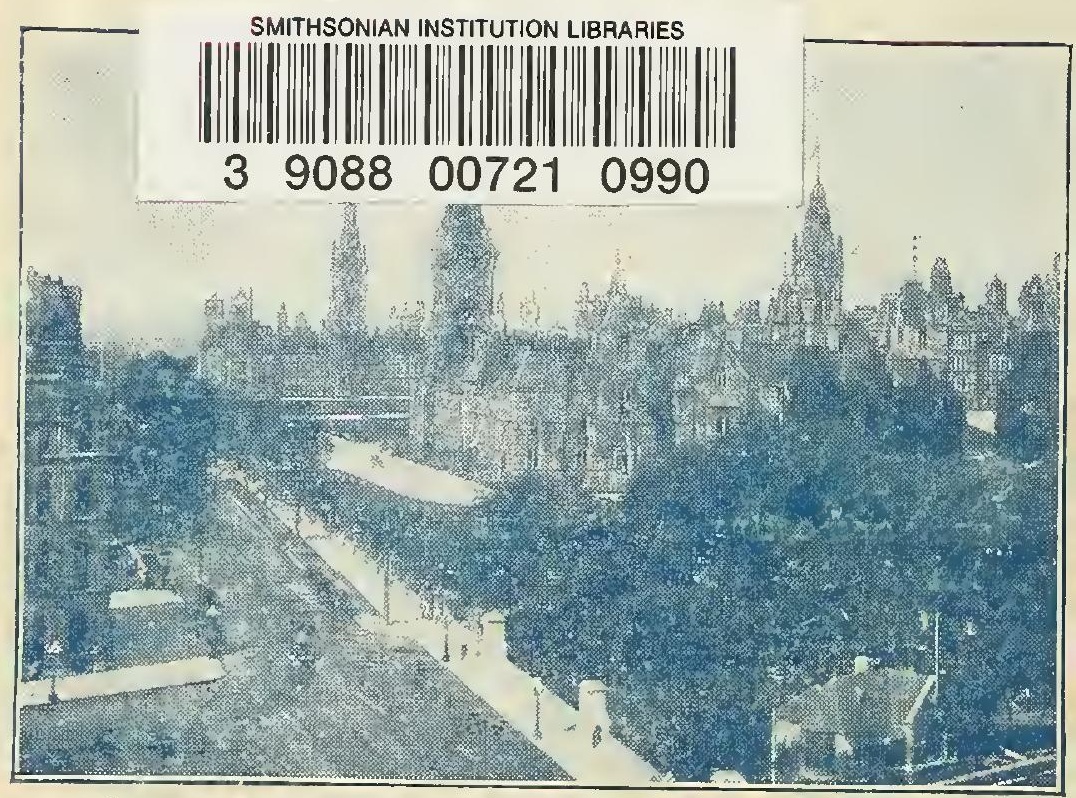

View of Pariiament Buildings from the Plaza

Jnly 55 miles irom Prescott. Opposite Ogdensburg,

N. Y., on the New Provincial Highway

The isost Deautiful City on the American Continent

with its Public Parks, Boulevards and Waterways

The Gateway to the Gatineau Hills, Province of Quebec, the Switzer land of Canada

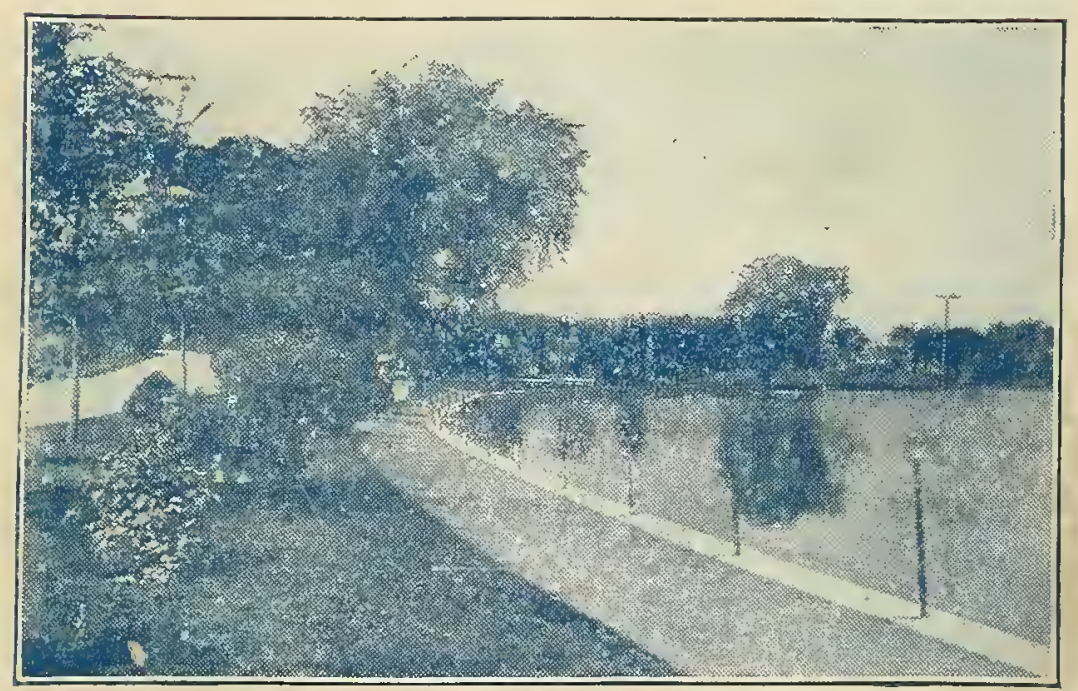

A Beauty Spot along the Driveway

Write the Secretary

Industrial and Publicity Commission

Touring Bureau, 26 Wellington Street

OTTAWA, CANADA
5296 SI BR
$03 / 94 \quad 198115$
4149 
